Jap. J. Smooth Muscle Res., 6, 51 55, 1970

\title{
What is Important of the Gastric Smooth Muscle in the Origin of Peptic Ulcer?
}

\author{
MINORU OI, M.D. \\ Department of Surgery, Jikei University, School of Medicine, Tokyo, Japan
}

First of all, I must tell you that my topic is not the one written in the program. Today, I talk about "What is important of the gastric smooth muscle in the origin of peptic ulcer?". The direct motive which had made us study the function of the smooth muscle is our intense concern that the motility of the stomach, as well as secretion, should play an important role in peptic ulceration. Therefore, the smooth muscle organ as the object of our study has been limited to the stomach only. And all the works have been concentrated into the direction of exploring the mechanism of peptic ulcertation. My talk today is also from this direction, but I hope that the attendant here who are not from the same research field could also feel a relationship or some principal interest.

\section{Mưcosal Rưle}

In Figure 1, you see new technical terms, that is, dual control mechanism, mucosal rule and muscular rule. All these were newly made by us. I will explain these terms first.

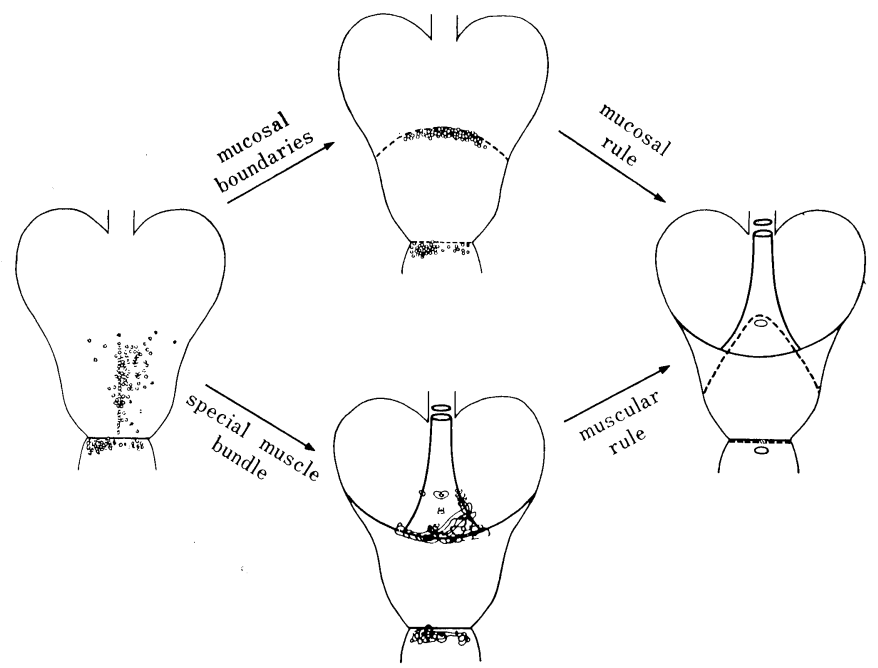

FIg. 1. Dual control mechanism by the mucosa and musculature upon occurrence of peptic ulcer. 
After the gastric resection, the stomach is opened along the greater curvature. In the diagram on the left, the real location of ulcers is plotted. The conventional knowledge that gastric ulcers occur at the lesser curvature, that duodenal ulcers at the most proximal duodenal portion and that gastric ulcers are sporadic while duodenal ulcers are concentric are well demonstrated in this diagram. However, what we regret is that there have been no rational explanation on these important facts. Now, by our histological observation one principle was found that gastric ulcers occurred near the boundary of the fundic gland area and pyloric gland area and that the duodenal ulcers occurred near the boundary of the pyloric gland area and duodenal gland area. And, furthermore, these ulcers were found to be on the side of the boundaries which were opposite the fundic gland area, namely, acidsecreting area. This relation is illustrated in the diagram at the top. The diagram at the buttom will be explained later in this talk.

Of 855 ulcers in total found in 640 gastric specimens, 829 or $97.0 \%$ were under this principle. We call this principle mucosal rule: "Peptic ulcers occur adjacent to the boundary of different mucosae and opposite the acid-secreting area." The mucosal rule points out that location or development itself of peptic ulcer is strongly controlled by the arrangement of the mucosal area. The conventional concept which knew no rule about the ulcer location is greatly forwarded here.

Now, shall we consider why such rule has been over-looked? The reason is simple. Because, it was not paid attention that the location of the fundo-pyloric mucosal boundary is quite variable among individuals.

Figure 2 shows how it varies. The right diagram shows the location of ulcers in the gastric specimens which was surgically resected. The middle diagram shows the location of mucosal boundaries in these specimens. The left one shows the mucosal boundaries in the specimens without ulcer or other gross lesion, namely, the normal stomach. Here, the similarity of the middle and left diagrams points out that the variation in the mucosal boundary is not due to ulcer formation but
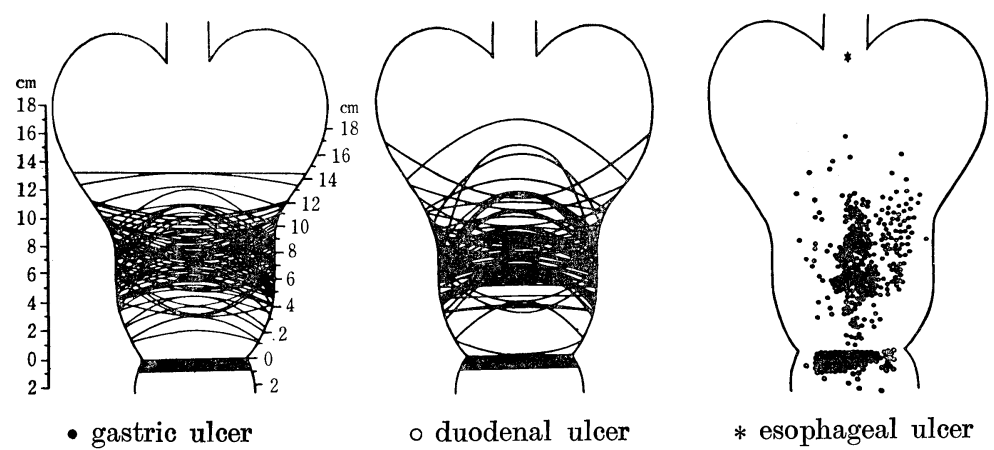

Fig. 2. Location of peptic ulcer in relation to mucosal boundaries. 
exists congenitally. The location of stomach ulcer varies, simply because the fundopyloric mucosal boundary varies individually. The location of duodenal ulcer is quite concentric, also because the pyloroduodenal mucosal boundary does not vary so grossly.

\section{Mưscular Rưle}

As the next step, I will speak about the ulcer and the musculature of stomach. Now the study was conducted with the entire muscle coats whose inner surface was exposed by removal of the whole mucosal layer.

The inner layer of the muscular coat consists of oblique bundles which are derived from the circular muscle bundle of the esophagus. These run obliquely in both anterior and posterior wall of the stomach and gradually disappear by fusing with the gastric circular muscle bundles. Among these bundles, ones which lie. nearest the lesser curvature were named "anterior medial oblique muscle bundle" and "posterior medial oblique muscle boundle." The circular muscle bundle to which these special oblique bundles are united was named "border circular muscle bundle". The bundle forming the thickest part of the pyloric sphincter was
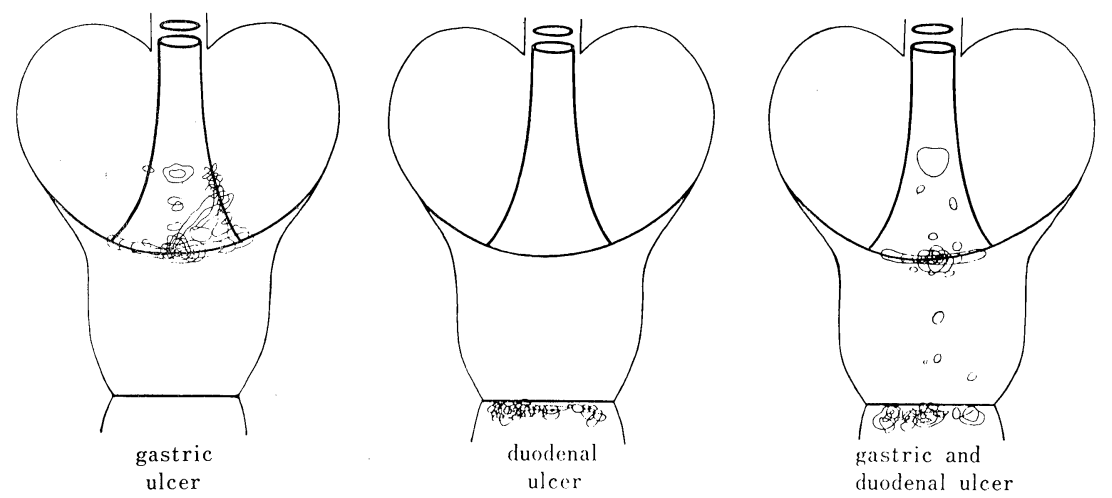

Fig. 3. Location of peptic ulcer in relation to special muscle bundles.

named "pyloric circular muscle bundle" (see Fig. 1 and Fig. 3). Now, there may arise a question why these are so special. The reason is that the kinetic strain is presumed to be especially strong along these bundles. The location of ulcers was observed now in relation to these special bundles and the result is as illustrated in Fig. 3. As shown in the left diagram, all the gastric ulcers, except a few, were found on the area enclosed by both medial oblique muscle bundles and border circular muscle bundle, or the area we call upper strain zone. The size and shape of ulcer should have a close relation with these special bundles, therefore, each ulcer was put down as realistic as possible. If all the ulcer had been put down together, the upper strain zone, especially its part of the posterior gastric wall would have 
turned just black. So much the ulcers were concentrated to the upper strain zone. How about duodenal ulcers? They also showed a certain strong tendency as you see in the middle diagram. They all occurred on the immediate vicinity of the pyloric cirular muscle bundle or the area we call lower strain zone. The combined gastroduodenal ulcers were found on the upper and lower strain zones, as illustrated by the right diagram. You may easily understand this. 259 of the toal 269 ulcers which were found in $211 \mathrm{~g}$ astric specimens examined in this study, or $96.3 \%$, were on the strain zones. This is the relation which we named muscular rule: "Peptic ulcers occur where the kinetic strain due to gastric motility is so great".

\section{Dúa Control Mechanism}

Thus, it is evident that the location of peptic ulcer is controlled not only by gastric mucosa but also by the gastric musculature.

This is the very relation which we have mentioned as "dual contol mechanism by the mucosa and musculature".

Now, to be more concrete in this regard, I will show the outcome obtained, when the feature found on the separated sheet of mucosa and that found on the musculature were put back. Fig. 4 shows the variety of the mutual relation exemplified in the real instances. The relation varies a good deal, because the location of the fundo-pyloric mucosal boundary and that of the border circular muscle bundle vary independent from each other. The relation between these two figures can be divided into 6 types. All the gastric specimens with ulcers in the

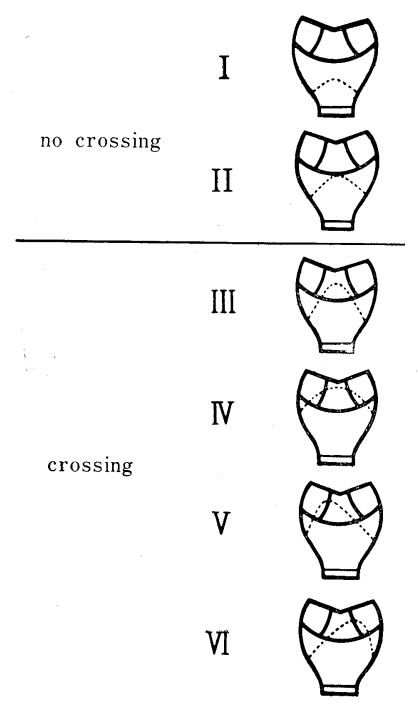

Fra. 4. Relationship between fundopyloric mucosal boundary and border circular muscle bundle, 
stomach, except for only one, belonged to the crossing type exclusively. This is quite logical because the dual control mechanism effects an ulcer only distal to the fundo-pyloric mucosal boundary and proximal to the border circular muscle bundle. This striking tendency makes a sharp contrast with the group of normal stomachs in which both non-crossing $(52 \%)$ and crossing types $(48 \%)$ are equally balanced.

\section{Discussion and Conclusion}

Conventionally, the systemic factor has been important in the pathogenesis of peptic ulcer. However, now in the light of dual control mechanism, the systemic factor appears subordinate to the local control factor. And it is deducible that the person whose stomach is in non-crossing type will not develop an ulcer, no matter how favorable is his systemic factor for ulceration.

Now, I will refer to the function of the smooth muscle which is supposed to be important as I mentioned in the beginning. But it is now clear that this importance is conditioned by the local anatomy. Dr. Tanaka representing my team will talk about the functional study, namely, the electromyography of the stomach done in our department in following paper. He must be, however, amply alert not to overlook the delicate local anatomy with which I am vitally concerned.

Concerning duodenal ulcers, we must consider the local factor again. There aer four types distinguished as to the types of relation between the pyloroduodenal mucosal boundary and the pyloric circular muscle bundle. However, these figures are so close to each other and not so variable that the different types have no essential distinction among themselves. Therefore, it is quite likely that the role of systemic factor is much straighter in the duodenal ulcer than in the gastric ulcer. And such a fact is quite an information, although not subversive, to me whose attitude is to put both gastric and duodenal ulcers on the same pathogenic mechanism. The United States has high incidence of duodenal ulcer and the importance of systemic factor is stressed in the United States. This is quite understandable. However, I hesitate and feel a controversy in taking up this importance when we face the gastric ulcer which is more frequently found in Japan.

Needless to say, hypersecretion of the stomach is a favorable background of peptic ulcer, and so is the hypermotility according to our elucidation. However, it is also true that not all the persons with hypersecretion or hypermotility has peptic ulcer.

Shall we often think the anatomy as well as physiology of the smooth muscle? 
Jap. J. Smooth Muscle Res., 6, 57 61, 1970

\title{
Smooth Muscle Function and its Relation to Disease of the Gastrointestinal Tract
}

\author{
HARRY A. OBERHELMAN, JR., M.D. \\ Department of Surgery, Stanford University School of Medicine, Stanford, \\ California, U.S.A.
}

The importance of motility disturbances that either produce pathological disease states or result from gastrointestinal lesions has been emphasized. The application of recently developed electrophysiological techniques in the diagnosis and understanding of gastrointestinal disease will enhance our therapeutic efforts in clincial medicine. Closer cooperation between physiologist and clincian is stressed.

Our knowledge of the physiology and pharmacology of gastrointestinal smooth muscle has been acquired primarily through the efforts of physiologist during the past five decades. Despite their contributions effective utilization of this knowledge in the diagnosis and understanding of gastrointestinal disease in man has emerged only recently. Since the alimentary tract of man consists of smooth muscle throughout except for the pharyngeal and anal ends, one can readily reoognize the importance of motility disturbances that either produce pathological disease states or result from gastrointestinal lesions. The magnitude of the problems we are faced with is attested to by the observation that digestive diseases are the leading cause of hospitalization in the general population, produce more days of hospitalization than diseases of any other body system, are the second major cause of days lost from work, and are the third leading cause of death in the United States. ${ }^{1)}$

It is expected that recently developed electrophysiological techniques will enhance our knowledge of gastrointestinal smooth muscle function in both health and disease thus improving methods of diagnosis and treatment in clincial medicine. Currently employed radiological methods of study including cine-radiography have yielded valuable information in esophageal disease. However, their use in gastric and intestinal disease is limited due to radiation hazards from long term exposure required for meaningful studies. The use of billooins, open tip tubes, and capsules to record intraluminal pressures has not been altogether satisfactory in interpreting gastrointestinal motility, particularly in the stomach. Although useful information of motor activity can be obtained in experimental animals by the use of silicon strain gauges, their use in man is limited for obvious reasons. Similarly, the quantitation of peristatlic or mechanical activity to elelctrical activity remains 
indeterminate and further observations utilizing all available methods of measuring smooth muscle function will be needed before the value of electrophysiologic techniques is realized. In addition, this must also include simultaneous measurements of emptying and transit times in order to accurately assess gastrointestinal motility. Despite the introduction of barium motor meal studies, radioisotope and dye dilution studies to measure gastric emptying, more accurate and physiological methods are needed.

Although it would seem convenient to catagorize disease conditions on the basis of neural (extrinsic or intrinsic), myogenic, humoral, vascular, or conduction disorders, current available knowledge frequently indicates a complexity or lack of etiological mechanisms involved. I will review briefly those disease processes including iatrogenic conditions of the alimentary tract that involve abnormalities in smooth muscle function and in which further knowledge may elucidate more clearly their etiological mechanisms, diagnosis, and specific form of therapy to be adopted.

Utilizing balloon methods and more recently open tip catheters, pressure studies of the esophagus have delineated characteristic patterns in achalasia, Changa's disease, diffuse spasm, scleroderma, and hiatus hernia. In addition, a zone of increased pressure at the gastroesophageal junction has been shown to act as a physiologic lower esophageal sphincter preventing regurgitation of gastric contents. The factors leading to incompetency of this sphincteric mechanism, particularly when displaced upwards as in hiatus hernia, remain controversial. Further studies are needed to assess the role of the phreno-esophageal ligament, changes in angulation between the esophagus and stomach, the sling fibers of the stomach, and variations in pressure above and below the diaphragm in maintaining a competent lower esophageal sphincter.

With the adent of cine-radigoraphy an increasing variety of esophageal motility disturbances have been observed in addition to the recognizable entities previously mentioned. The cause of these disorders is less well understood using conventional methods of study. Since there have been few electrical measurements of esophageal smooth muscle in man, it may be anticipated that future studies may elucidate the mechanisms of these disorders as well as leading to more appropriate therapy. Is it not possible that our knowledge of the gastro-esophageal sphincter might also be furthered by such studies?

The application of electrical activity to smooth muscle function of the human stomach in health and disease has received impetus as a result of the development of new electronic techniques to retrieve the signal. The use of intragastric or cutaneous electrodes permit long term studies in patients with gastric disease and postsurgical disturbances without operative implantation of electrodes. Observations with multiple electrodes of the frequency of the gastric electrical rate and its statistical variations have proved useful in detecting vagal denervation, the response to feeding, reverse peristatlic activity, and the presence of multiple 
pacemakers or conduction paths in the stomach of man. ${ }^{2)}$ However, the quantitation of mechanical or peristaltic activity to the slow potential variations is yet to be determined. Suitable signal processing to measure spike artivity and amplitude variation may be expected to provide a better correlation in this regards.

Since intraluminal pressure measurements of the stomach are often unsatisfactory, it is hoped that correlative studies using electrical measurements and cine-radiography will increase our knowledge in the diagnosis and treatment of gastric disease as well as our understanding of the normal physiology of gastric motility, the control of gastric emptying, the function of the pylorus, and gastrin release. It has been suggested that the release of antral hormone, gastrin, may be related to antral peristalsis. ${ }^{3)}$ The factors controlling gastric and intestinal motility are complex and involve the integrated action of the extrinsic autonomic nerves, the intrinsic nerve plexus, the inherent properties of smooth muscle, and humoral agents.

Disorders of the stomach that may result from abnormal smooth muscle activity include duodenal and gastric ulcer, gastric neuropathies, and post-surgical syndromes that interrupt vagal innervation, gastric peristalitic activity, and pyloric function. What evidence exists for increased motor activity in patients with duodenal ulcer? The role of the gastric antrum and impaired gastric emptying as postulated by Dragstedt remain controversial in the etiology of benign gastric ulcer. ${ }^{4}$ Studies of gastric emptying have been conflicting indicating the inadequacies of the tests employed. It would seem that studies of electrical activity as well sa improved motor meal tests may clarify the role of stasis in the etiology of gastric ulceration.

There have been a number of reports in the literature emphasizing gastric motility disorders in diabetes mellitus. Early recognition of such defects may be anticipated from electrogastromygoraphy in addition to the selection of appropriate therapy. Abnormalities of gastric motility in other systemic diseases require further study as well as in intrinsic diseases of smooth muscle.

Our understanding of gastric motor disturbances following surgery of the stomach remains limited. The impairment of gastric emptying following vatogomy has been characterized electrically as showing a dysrhythmia or arrhythmia and a reduced incidence of fast activity. Could this be a better index of the completeness of vagotomy than the secretory response to insulin hypoglycemia? The relative merits of the various gastric drainage procedures to relieve stasis after vagotomy are poorly understood. Accurate gastric emptying studies and the effects of pyloroplasty or gastro-jejunostomy on the electrical activity of stomach smooth muscle function can be expected to elucidate the beneficial effects of these procedures as well as indicating the most effective one.

The disturbances in motor activity following the various resective procedures of the stomach as well as wedge resection or gastric transection also deserve further 
study. In order to understand the nature of such disturbances it is necessary to delineate the pacemaker and conduction mechanisms of the stomach. As our knowledge of these functions is acquired, operative procedures on the stomach will be more intelligently selected to treat gastric disease.

The intestinal tract probably provides the greatest challenge in terms of the development of monitoring techniques as well as the vast number of diseases both functional and organic that involve the small and large intestine. It is not infrequent that many disease states produce digestive disturbances of a motor nature prior to their detection by current radiological methods. Electrical and intraluminal pressure studies may lead to earlier diagnosis and more effective medical or surgical therapy. One may expect specific abnormalities of conduction in those diseases that involve smooth muscle such as granulomatous colitis, radiation enteritis, amyloidosis, and collagen diseases. The detection of impending necrosis or tissue death as seen in vascular insufficiency secondary to thrombosis, embolism and strangulation obstruction should prove amenable to electromyography. The era of transplantation will soon extend clinically to the gastrointestinal tract and it may be predicted that electrical measurements will be an important index of early immunological rejection. There is an immediate need for animal studies in this new and exciting area.

In addition to electromyography there is a need for accurate assessment of transit times or peristaltic flow rates. Electrical and pressure waves have not proved a reliable index of propulsion. The aboral transport of food is more closely related to the pressure gradient between proximal and distal segments than to the activity of any single type of contraction. This gradient is probably related to the progressive decrease in electrical activity as one descends to the terminal ileum. Radiological studies are often unsatisfactory due to the unphysiological characteristics of barium sulfate. Further studies are needed in those motility disturbances associated with bowel resection, interposed iso- and anti-peristalitic segments, malabsorption syndromes, inflammatory diseases, and those disorders secondary to various endocrine and neurogenic abnormalities. Illustrative of the latter is Hirshsprung's disease where the aganglionic bowel extends for from the rectum upward for variable distances. Electromyography by delineating the defective bowel segment should aid in operative therapy. The value of posterior sphincterotomy recently advocated in the surgical management of localized Hirshsprung's disease needs confirmation.

Other areas deserving further investigation are the intimate mechanisms and abnormalities of the ileo-cecal valve and anal sphincters. Is the function of the ileo-cecal sphincter to prevent regurgitation or to regulate intestinal flow into the colon or both? Loss of the ileo-cecal sphincter frequently alters normal bowel function. Further studies of this sphincter are needed to evaluate its role in intestinal transit and absorption. Similarly, electrical and pressure measuremnts of the 
terminal colon are needed to assess the function of the internal and external anal sphincters in maintaining continence.

I have obviously not been able to cover all of the disorders in smooth muscle function of the gastrointestinal tract and its appendages in this biref review. It is hoped that this will stimulate closer cooperation between physiologists and clincians in solving some of the perplexing motility disorders of the alimentary tract.

\section{References}

1. Grossman, M.I.: Digestive disease as a national problem. I. An account of a conference on digestive disease as a national problem. Gastroenterology 53:689-696, 1967.

2. Nelsen, T.S., Eingenbrodt, E.H., Keoshian, L.A., Bunker, C. and Johnson, L.: Alterations in muscular and electrical activity of the stomach following vagotomy. Arch. Surg. 94: 821-835, 1967.

3. Oberhelman, H.A., Jr., Rigler, S.P. and Dragstedt, L.R.: Significance of innervation in function of gastric antrum. Amer. J. Physiol. 190: 391-395, 1957.

4. Dragstedt, L.R.: A concept of the etiology of gastric and duodenal ulcer. Caldwell Lecture, 1955. Amer. Journ. of Roent. Radium Therapy and Nuclear Medicine 75: $219-229,1956$. 
Jap. J. Smooth Muscle Res., 6, 63 65, 1970

\section{Action Potentials of Visceral Smooth Muscles and Their Relation to Mechanical Activity}

EMIL BOZLER, Ph.D.

Department of Physiology, The Ohio State University, Columbus, Ohio, U.S.A.

Probably all normal contractions of smooth muscle are associated with changes in membrane potentials. That these potentials control contraction is suggested by the fact that depolarization induced by chemical agents or electric current induces contraction. One might, therefore, hope to find a simple relation between the action potentials and mechanical response. However, this is not true for any type of muscle, and particularly so for smooth muscle.

The potentials of smooth muscles associated with conducted responses vary more than in other types of muscle and differ in various organs of the same species and in the same organ of different species. We may distinguish three types (cf. 4). (1) The conducted potentials of the uterus and the intestine of the guinea pig are spikes. The strength of contraction varies with the number and frequency of the spikes. (2) The ureter and stomach of some species give plateau potentials differing from those of cardiac muscle only by the longer duration of the plateau. Prolongation or shortening of the plateau by drugs influence the strength of contraction. (3) The gastrointestinal tract of most species has plateau potentials on which spikes are superimposed. Conduction must be assumed to be due to the plateau potentials because it continues when the spikes are absent, as is true during weak contractions.

Alvarez and others (1) observed that electrical activity of the rabbit's intestine continues when there are no visible movements. This activity consists of plateau potentials. The strength of contraction is determined chiefly by the spike potentials superimposed on the plateau.

It should be noted that the shape of the action potentials recorded with external electrodes depends greatly on the distance between the electrodes. If this distance is small, compared with the length of the excited region, the records are the first derivative of the membrane potential. In the intestine of most species such records show $\mathrm{R}$ and $\mathrm{T}$ waves like those of cardiac muscle demonstrating that they are due to plateau potentials. With more widely separated electrodes the potentials are recorded as smooth diphasic, often sinoid waves. The shape of these waves depends in part on conduction velocity because the length of the active region diminishes as conduction becomes slower. It is important to realize that in 
work with implanted electrodes the distance between electrodes is generally large. The potentials recorded under these conditions are often described as slow potentials. However, the records of the action potentials obtained with this method agree completely with the assumption that they are basically due to plateau potentials.

Recent studies with intracellular electrodes generally confirm the results obtained with extracellular electrodes. It is surprising, however, that conducted potentials with a rise time of about a second have been recorded with intracellular electrodes. Because these potentials are slower even than those which can be obtained with suitable external electrodes, it seems likely that the fibers have been injured by the impalement. This assumption is supported by the small size of the potentials. It appears that what is described in the literature as "slow potentials" are either plateau potentials recorded with widely separated electrodes or are obtained from injured fibers, or are non-conducted potentials.

The combination of plateau potentials and spikes, as observed in the gastrointestinal tract of animals such as the rabbit, cat, and dog, is unique for smooth muscle. The simplest explanation for this phenomenon is that two distinct ionic mechanisms come into play, a hypothesis which is strongly supported by recent experiments (6). They suggest that the mechanism may be similar to that in cardiac muscle in which voltage clamp experiments have clearly shown that depolarization is caused by two separate mechanisms perhaps involving 2 separate channels, a fast movement of $\mathrm{Na}$ and a delayed influx of both $\mathrm{Na}$ and $\mathrm{Ca}$. Perhaps in some smooth muscles Ca enters the fibers in brief bursts, thereby producing spikes, while in other muscles this process is slow and is a component of the plateau potential as in cardiac muscle.

Rhythmic contractions of the intestine usually are rather weak. Much more powerful contractions are observed during reflex peristalsis, which can be induced by a bolus or obstruction of the lumen. In the dog, action potentials of these contractions are like those during ordinary rhythmic activity, except that the spike discharge is increased, but in the ileum there is complete electrical silence at the height of contraction (3), probably because the fibers are in a prolonged state of depolarization. Such a condition can be produced and has been directly demonstrated by microelectrode studies in the colon of the guinea pig by stimulating parasympathetic nerve fiber at a high frequency (5).

Besides the rapidly rising conducted action potentials just described, there are non-conducted changes in membrane potentials which are also associated with mechanical changes. In the ureter it has been shown that the pacemaker potentials are closely paralleled by changes in tension (2). In the colon of the guinea pig changes in tonus are accompanied by changes in resting potential (5). 


\section{References}

1. Alvarez, W.C.: The mechanics of the digestive tract (2nd ed.) New York: Hoeber, 1928.

2. Bozler, E.: The activity of the pacemaker previous to the discharge of a muscular impulse. Am. J. Physiol. 136: 553-561, 1942.

3. Bozler E.: Peristalsis of the intestine. Am. J. Physiol. 157: 2-8, 1949.

4. Bozler, E.: Smooth muscle. In: Muscle as a tissue. Edited by Eodahl and Horvath, McGraw-Hill Book Co., 1962.

5. Gillepsie, J.S.: The electrical and mechanical responses of intestinal smooth muscle cells to stimulation of their extrinsic parasympathetic nerves. J. Physiol. London. 162: 76-92, 1962.

6. Liu, J., C.L. Prosser, and D.D. Job: Ionic dependence of slow waves and spikes in intestinal muscle. Am. J. Physiol. 217: 1542-1547, 1969. 
Jap. J. Smooth Muscle Res., 6, 67〜68, 1970

\title{
A Concept of Motor Control by the Pacesetter Potential in the Stomach and Small Bowel
}

\author{
CHARLES F. CODE, M.D., Ph.D., JOSEPH H. SZURSZEWSKI, Ph.D. \\ AND KEITH A. KELLY, M.D. \\ Mayo Clinic and Mayo Foundation, Rochester, Minnesota, U.S.A.
}

Our thesis is that the rhythmically recurring, omnipresent fluctuations in potential, occurring particularly in the longitudinal muscle coats of the stomach and small bowel and identified there as the slow wave, the basic electric rhythm (BER), or the pacesetter potential (PP), exercise control of the motor activity of these organs. If at any one instant the electrical activity of the stomach or small bowel could be stopped or frozen in place, each slow wave (PP) would be standing or fixed in the wall and would extend over a distance which may be termed its "cycle length" or "wavelength" in that particular region. We propose that the portion of stomach or bowel beneath each cycle of the PP represents a physiologic motor segment and that the slow wave prescribes the dimension of the segment and controls the nature of motor activity which may occur at any one instant within it.

The frequency of the PP is uniform over the corpus and antrum of the stomach but displays a declining gradient over the small bowel. Velocity of propagation (conduction) is faster as the wave passes caudadward in the stomach but slows caudally over the small bowel. Thus, wave or cycle length (wavelength=velocity (frequency) increases as the PP passes distally in the stomach but diminishes as it proceeds analward in the small bowel.

The wavelengths of the PP and thus the length of motor segments of the stomach lengthen caudadward while those in the small bowel shorten. We have not determined the total number of cycles of the PP which may, at any one instant, reside in the stomach or over the entire length of the small bowel. This number is likely relatively fixed in different species. It represents the total number of individual contractions which could occur in the stomach or in the small bowel at any one instant.

The PP controls the motor activity within its wavelength because action potentials which accompany or initiate contractions occur only over a limited portion of the wavelength. Each PP cycle length or motor segment thus has associated with it only one burst of action potentials and so only one band of contracting fibers. The exception to this rule lies in the ileum. Here as wavelength becomes short and action potentials occur over the entire wavelength, 
contracting segments may fuse to produce broad bands of simultaneously contracting fibers involving many segments.

The PP fixes the maximal frequency of contraction and in its caudad migration synchronizes and coordinates contractions, determining their frequency, their velocity of propagation, and the width of the contracting band of muscle fibers. While the PP is clearly not the "computer" which programs gastrointestinal motility, it does appear to be the instrument through which motor patterns are consummated.

This investigation was supported in part by Research Grant AM-2015 from the National Institutes of Health, Public Health Service. 


\title{
Ionic Basis of Intestinal Potentials Conduction of Spikes and Slow Waves
}

\author{
C. LADD PROSSER, Ph.D. \\ Department of Biology, University of Illinois, Urbana, Illinois, U.S.A.
}

Fibers of the longitudinal layer of cat duodenum or jejunum show spontaneous rhythmic slow waves, five to seven seconds duration; spikes, when present, are at the wave peak. Circular fibers show only spikes; however, if attached to longitudinal fibers, slow waves are detectable. A pacemaker or prepotential can be seen at the beginning of spikes and at the peak of a slow wave. Contractions occur only when spikes are present.

Slow waves are reduced in amplitude in low sodium and may be abolished in sodium-free medium, also by ouabain $\left(10^{-7} \mathrm{M}\right)$. Spikes are lost in calcium-free medium, abolished by $\mathrm{Mn}^{++}$and $\mathrm{Co}^{++}$. Neither potential is effected by tetrodotoxin. Efflux of radioactive sodium shows a peak at the downward phase of slow wave, and this efflux peak is abolished by ouabain, passive influx of sodium occurs in rising phase. A peak of calcium flux (especially influx) appears at the top of the slow wave where spike occurs. Potassium fluxes are relatively uniform, but efflux may peak near the trough of the slow wave. Slow wave amplitude is enhanced when sodium is iontophoresed inside a fiber; no such effect occurs with potassium electrodes and similar current pulses.

Frequency of slow waves is highly temperature-sensitive, is reduced by $\mathrm{CN}$, DNP and hypoxia. Cell membranes contain an Na-K ATPase. Spike amplitude increases with elevated calcium over a range where resting potential is little altered; longitudinal fiber spikes increase in porporition to $\log \mathrm{Ca}_{o}$ up to $5.0 \mathrm{mM}$ $\mathrm{Ca}^{++}$while circular fiber spikes show maximal amplitude at $1.8 \mathrm{mM}$ and then decrease. In high (3 times normal) calcium, especially when $\mathrm{Na}$ is low, lingitudinal fibers show bursts of high frequency spikes.

It is concluded that slow waves result from a rhythmic sodium pump, that the active phase is polarization rather than depolarization, and that the pacemaker reaction is in an energy-delivering step, possibly a redox pump. Spikes (and probably their prepotentials) result from calcium influx or release. The longitudinal fiber membrane is, therefore, a mosaic of patches for active sodium efflux and for calcium conductance change.

Slow waves are synchronized around a ring in intact intestine and are propagated longitudinally. Isolated strips of longitudinal muscle show no propagation, and if longitudinal cuts are made through the circular layer in intact 
segment, slow wave propagation ceases. Slow waves are not detected in underlying circular muscle if connecting strands between the two layers are cut, but slow wave spread is not abolished by ganglionic blocking drugs. Slow waves are detected in circular muscle if longitudinal fibers are adjacent and they are undiminished if the cricular fibers are depolarized by potassium. Hence, the spread of slow waves from longitudinal to circular appears to be electrotonic along the connecting strands which occur at much shorter separations than the space constant. In rings of isolated circular muscle, spikes are conducted in a spiral pattern in fiber bundle, but in longitudinal muscle with slow waves, no conduction of spikes could be detected. When ganglionic plexuses are blocked or allowed to degenerate, spiking is enhanced and slow waves are more regular.

It is concluded that slow waves synchronize spiking and, hence, contractions by bringing cell membrane potentials below the zone of firing, and thus the longitudinal is the pacemaker layer. Circumferential synchrony and longitudinal conduction require electrotonic cycling via the circular fibers and connecting muscle strands. The relation between slow waves and the conduction of spikes in circular layer remains unresolved. Both layers are under some inhibitory influence from neurones of the myenteric plexus. 


\title{
Electrophysiological Studies of Smooth Muscle by Various Recording Methods
}

\author{
TADAO TOMITA, M.D. AND HIROSI KURIYAMA, M.D. \\ Department of Physiology, Kyushu University, Faculty of Medicine (Dr. Tomita) \\ and Faculty of Dentistry (Dr. Kuriyama), Fukuoka, Japan.
}

The first part of the paper will be read by Dr. Tomita. The sucrose-gap method is widely used for recording the electrical activity in smooth muscle. The recording is possible because of cable-like properties of the tissues. In order to study the passive (membrane resistance) and active (non-propagated spike) properties of the membrane, in the guinea pig taenia coli and ureter, the double sucrose-gap method was employed, one gap being for recording and the other gap for applying current pulses. The responses from the central part of the tissue which was exposed to physiological saline was also recorded with an intracellular electrode. The results were in agreement with the idea of cable-like properties, but they also indicated some effects of sucrose immersion on the membrane properties.

The second part of the paper will be read by Dr. Kuriyama. The main theme is the electrophysiological studies of the guinea pig stomach. As experimental methods, combinations of microelectrodes, single sucrose-gap and double sucrosegap methods were used to investigate the membrane properties of the stomach smooth muscle.

The electrical activity of the longitudinal muscle showed quite different features from those observed in the circular muscle. Furthermore, various parts of the stomach, namely fundus, curvatures and pylorus also showed different electrical features from each other.

Various electrical properties of the stomach smooth muscle, e.g., membrane potentials in resting and active states, slow wave, conduction velocity, chronaxie, membrane resistance measured by intra- and extracellular stimulating methods and characteristic constants of the membrane will be described.

Furthermore, nervous factors influencing the membrane activity of the stomach will be discussed from the points of view of drug action on the muslce membrane. 


\section{Interpretation of the Electromyogram of the Small Intestine as recorded wih External Electrodes}

MOLLIE E. HOLMAN, D. Phil.

Department of Physiology, Monash University, Australia,

The 'basic electrical rhythm' or the slow waves of the small intestine are the most prominent feature of electromyograms taken with large external electrodes. On the other hand, intracellular records from segments undergoing active contraction are dominated by the occurrence of much faster 'spikes' of irregular amplitude and time course. An attempt will be made to account for the patterns of activity observed with external electrodes in terms of what is known of the membrane properties of intestinal smooth muscle.

The slow components of the electromyogram are prodably due to the synchronous depolarization of large populations of smooth muscle cells of the longitudinal layer. This depolarization can spread passively to the circular layer (Kobayashi, Nagai \& Prosser, 1966). It may be generated by one or both of the following processes:

1. Rhythmic fluctuations in membrane potential which are an intrinsic property of the longitudinal layer of smooth muscle. The frequency and synchronization of these waves would appear to be independent of the membrane potential of the smooth muscle. They may reflect a genetically determined property of the cell's metabolism in the form of a rhythmically active electrogenic ion pump. Synchronization might be achieved by the movements of ions in some other material across regions of membrane fusion between neighbouring cells.

2. Rhythmic release of excitatory or inhibitory neurotransmitters from the ganglion cells of the myenteric plexus.

Slow depolarizations observed with intracellular electrodes may also have a component which is due to the generation of action potentials at sites up to 1 or $2 \mathrm{~mm}$ distant from the recording electrode which are 'smoothed out' by the passive electrical properties of the tissue.

The diphasic or triphasic 'spike' components of the electromyogram recorded with large external electrodes indicate the generation of action potentials which may only be conducted for short distances.

Action potentials are probably the electrical events which trigger contractions. 


\section{Reference}

Kobayashi, M., Nagai, T. and Prosser, C.L.: Electrical interaction between muscle layers of cat intestine. Am. J. Physiol. 211, 1281-1291, 1966 
Jap. J. Smooth Muscle Res., 6, 75 77, 1970

\section{Electronic Modeling of the Gastric and Small Intestinal Pacemakers and Conduction Mechanisms}

THOMAS S. NELSEN, M.D.

Department of Surgery, Stanford University School of Medicine, Stanford, California, U.S.A.

It has long been recognzied that the effective propulsive activity of the stomach and small intestine depends upon peristaltic waves of sufficient strength or magnitude to propel a portion of the intraluminal content in the aboral direction with each wave. Timing and synchronization of these waves is of distinct importance in the effective propulsion of the intestinal content. In general, the stomach does not demonstrate a gradient of rate from the oral to the aboral part; in contrast, the small intestine shows a declining gradient of rate of contraction from the duodenum to the ileum. Whether a gradient of rate is present or not, it seems clear that the pacemaker and conduction mechanisms control the timing of peristaltic waves. The coupling of the omnipresent regular electrical slow waves to the mechanical contractile apparatus of the smooth muscule is variable in amplitude from essentially no coupling (quiescent condition) to full coupling (maximal contraction).

Specialized pacemaker tissue has not been found in the duodenum or stomach in the mammalian species studied in detail; it would appear that the pacemaker area dominates simply because its intrinsic rate is higher, since in coupled systems higher order pacemakers eventually dominate. Thus, one is faced with a system in which the pacemaker function, the conduction function and the contractile function are all in the same system of smooth muscle cells. The pacemakers of the species studied most carefully, the dog, are extremely stable and in the case of the duodenum, altered little in rate by changes of the physiologic state relating to the gastrointestinal tract. It is probable that the conduction velocity varies with the time-history of the electrical activity in the particular zone, the particular portion of the organ in question, the nutritional state, the temperature and other physiological variables. It seems reasonable to assume that variations in conduction velocity, if of correct polarity and sufficient magnitude, serve to smooth irregularities in the periodic electrical and mechanical behavior of the muscle.

In an attempt to simplify and unify the concept of propagated electrical activity in the stomach and intestine, one can view each system as a chain of biological clocks or oscillators coupled by the propagation of current from one 
to the next along anatomic paths; the higher order clocks dominating and acting as pacemakers. One can also view the contractile mechanism as a series of similar biological oscillators which are not free running (i.e., in a hold-off state) and require external energy from the free running oscillator for transformation to the active (oscillating) state. Thus, the nature of the coupling mechanism becomes of primary importance when one considers the mechanism of action of this chain of oscillators in the hold-off state. This parallel mechanical chain can be viewed as a relaxation cable.

The mathematical model chosen is a generalization of the van der Pol equation, an equation which was originally derived as the simplest equation to represent relaxation oscillations in certain electronic systems. This type of equation is of considerable importance in biology, since the oscillation is dependent upon some type of energy source. It is capable of producing a wide range of waveforms. The van der Pol equation has been generalzied by Fitzhugh, utilizing Lienard's transformation with added terms, so that it demonstrates most of the phenomena observed in excitable tissue, i.e., single impulses, trains of waves, thresholds, refractory states, etc. In fact, the generalized van der Pol equation is capable of duplicating the behavior of the Hodgkin-Huxley equation model in most respects, lacking delay phenomena and certain other features. This lack stems from the simplification of reducing the four Hodgkin-Huxley variadles to the two variadles of the van der Pol equation. The geometrically unrealizable four dimensional phase-space representations necessary to depict the complete Hodgkin-Huxley equations are thus reduced to two variables which can be plotted with ease as phase-plane diagrams, the ordinate representing refractoriness and the abscissa

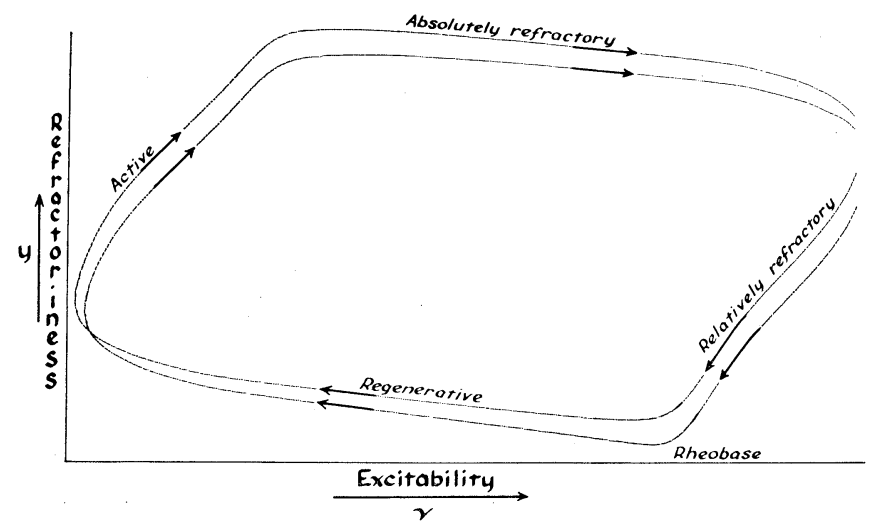

Fra. 1. Phase-plane plot of two single waves generated by a Fitzhugh oscillator with differing simulated resting membrane potentials. The resting value of membrane potential and beginning of the wave is at the dot. Trajectory is clockwise, $V=$ dependent variable; $y=$ first derivative function of $v$. The functional state of the equation at any point in its cycle can be inferred from the labels. 
excitability. In this representation, the trace is a trajectory which can be timemarked and readily interpreted in physiologic terms (See Fig. 1).

It would appear that the electrical slow waves generated by the smooth muscle of the stomach and intestine fall within the range of the van der Pol equation usually not treated in textbooks $(\varepsilon=2$ to 10$)$. Previous experience with a simple form of this model has yielded simulation of the intestinal gradient and duplication of the electrical and mechanical phenomena observed after partial and complete transection of the small intestine. The properties of coupling, entrainment, and statistical variation in the intestinal rate were well duplicated. Some insight concerning the coupling of electrical to mechanical activity was gained with this model and a prediction of the rate changes with peristaltic activity and stretch has been confirmed biologically.

The application of this same model to interpretation of surgical experiments upon the stomach appears valid. The stomach represents a somewhat different case of the coupling of relaxation oscillators with stability margins and degree of coupling differing from those in the small intestine. Complete entrainment is the normal situation. Alterations of rhythmic phenomena observed in the stomach after transection can be explained on the basis of the chain oscillator model, even though certain of the experimental results differ from the small intestine. 


\title{
Automaticity of the Alimentary Tract: Observation on the Fetal Alimentary Tract, the so-called Ganglion-free Intestine and the Anastomosed Organ
}

\author{
SHUHEI TAKITA, M.D. \\ Department of Surgery, School of Medicine, Tokushima University, Tokushima, \\ Japan
}

The present study deals with some of the basic problems related to the pathophysiology of peristaltic conduction following anastomosis of smooth muscle organs and the role of the nerve elments in the regulation of peristaltic direction.

The postoperative motility of the anastomosed digestive tract ${ }^{1)}$ was observed in 11 patients well recovered following esophago-gastrostomy. The motility of the tract was recorded selectively from above and below the anastomotic line by means of the double-balloons technic. We can see large esophageal contractions are immediately initiating a series of great contractions of the anastomosed duodenum, and after the Billroth I type gastro-duodenostomy, gastric peristalsis with 20second rhythm of the stomach initiating strong duodenal contraction. There is evidently an intimately cooperating pattern of movement in the digestive tract above and below the anastomotic scar.

To clarify the role of intramural nerves in the transmission of contractions across the anastomosis, the process of the nervous regeneration at the anastomotic stomach was studied by a series of histological observations of the experimental anastomosis of the esophagus, the small intestine and the proximal colon. ${ }^{2,3}$ The histological veiw (Fig. 1) of the anastomotic scar between two smooth muscle organs shows that a fine regenerated nerve fiber is seen passing through the scar from the Auerbach's plexus of one side to the plexus of another side. Generally, the regeneration of these nerve fibers was completed in approximately sixty days after anastomosis.

Although the conduction of motor excitation across the anastomotic line is likely to be due to this early nerve regeneration connecting both sides, another possible mechanism involved in the propagation is mechanical factor. It is still questionable, therefore, whethere these regenerated nerve elements alone are responsible for the transmission of all the impulses between the two tracts.

To follow up the changes in the mode of peristaltic conduction following anastomosis, ${ }^{4,5)}$ action potentials were recorded postoperatively from the anastomosed intestines of 140 rabbits (Fig. 2). 


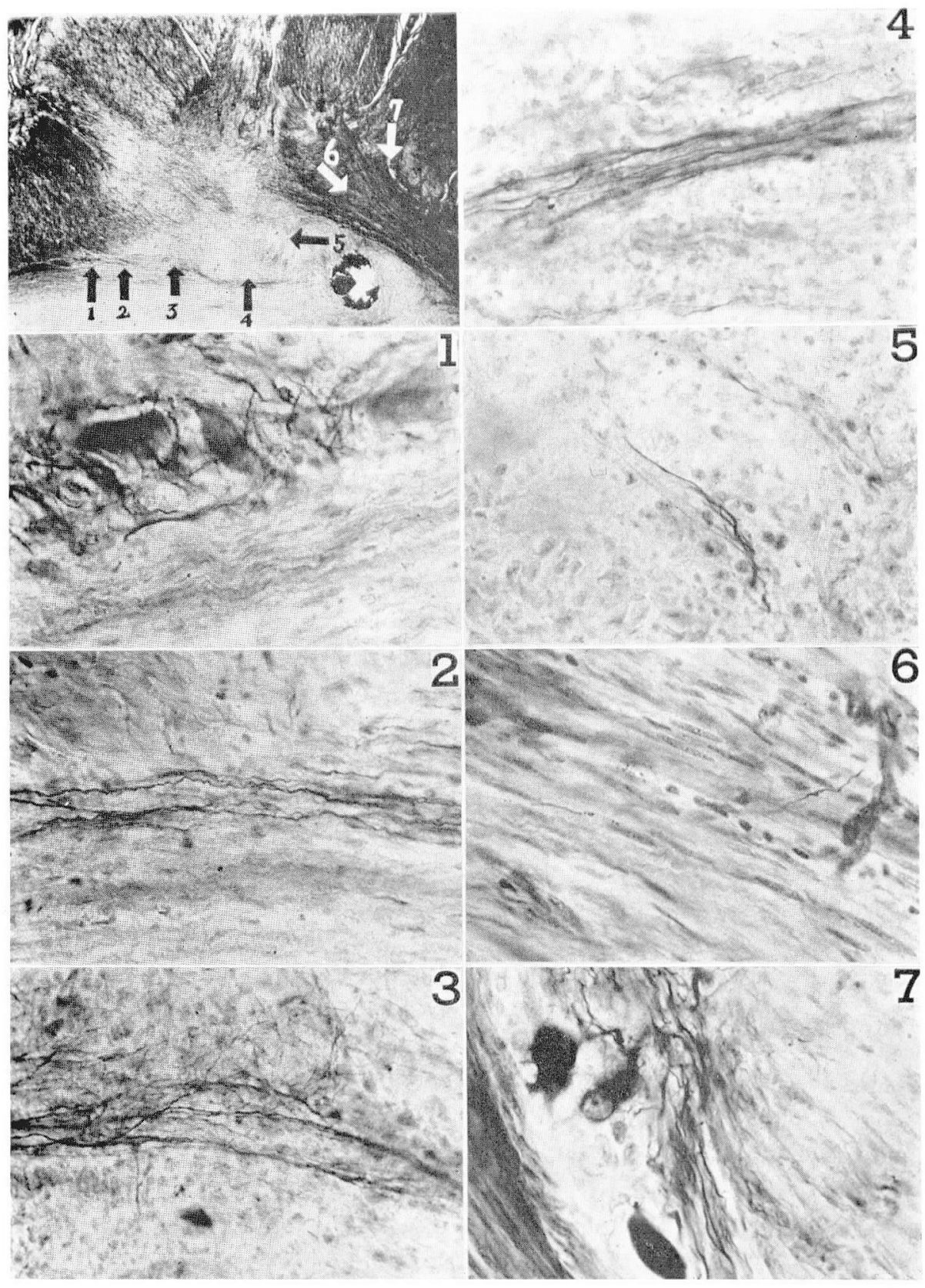

FIG. 1. Histology of anastomotic scar, 90 days after enteroenterostomy. Each photonumber of regenerated nerve-fiber corresponds to the arrow number in the left upper picture showing low-magnification histological view of scar between two intestinal walls of rabbit. 


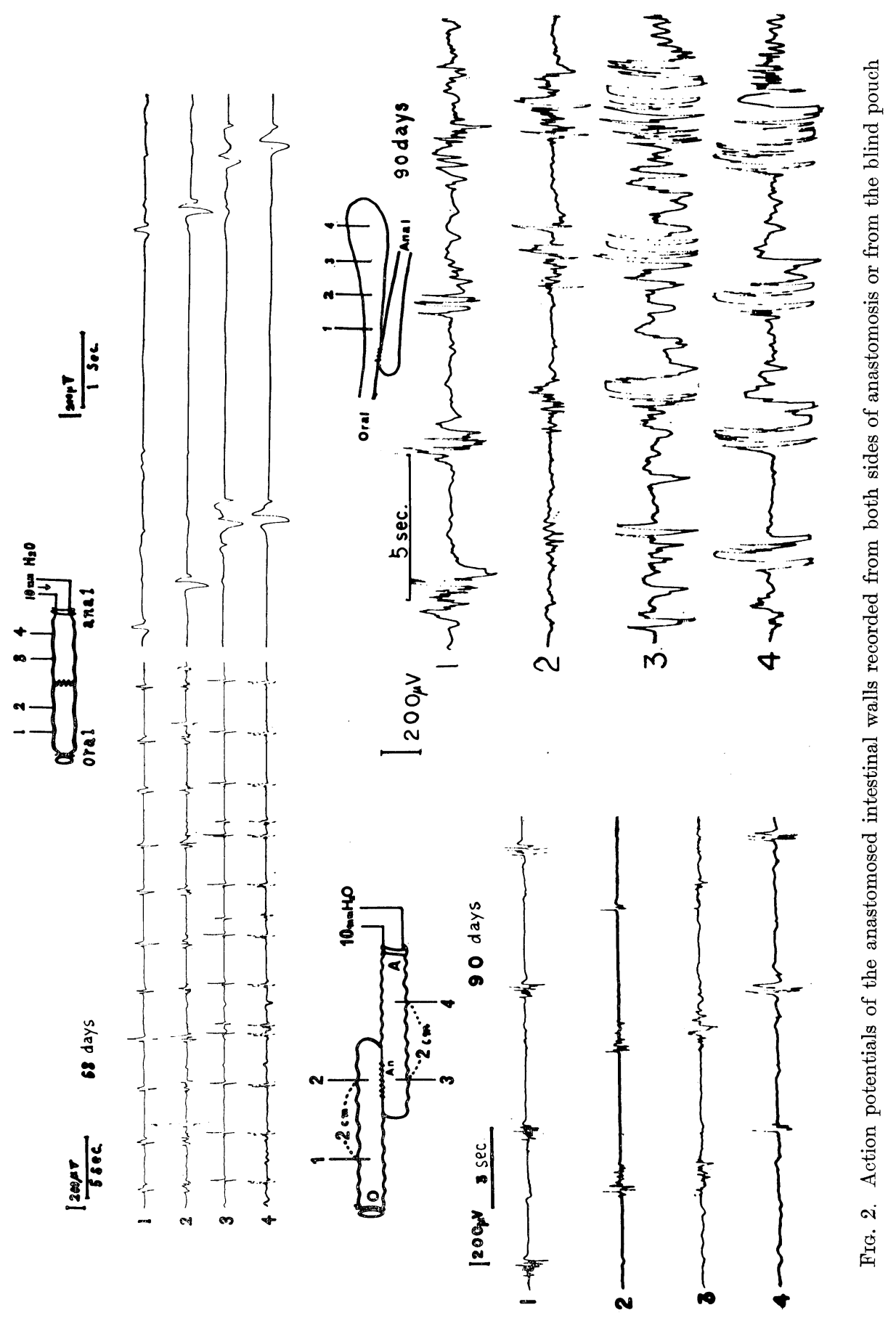


A segment of the anastomosed intestine was excised and suspended in a bath filled with Tyrode solution according to a modified Trendelenburg's method. The action potentials were recorded from the intestinal wall by means of 4 electrodes, each connected to an amplifier of an electroencephalographic recorder. Corresponding to the contractions at 4 electrode sites, the spike bursts were respectively recorded. However, the spike bursts recorded seven days after an end-to-end anastomosis indicated no regular periodicity and no propagative character. Two months postoperatively, almost all the spike bursts are indicative of peristaltic propagation from oral to aboral across the anastomotic line in a similar fashion as in the normal intestine.

With a side-to-side anastomosis, the pattern of action potentials gradually returned to normal within two months. It is known that a side-to-side anastomosis frequently gives rise to a formation of a blind loop or pouch and results in a considerable stagnation of the content associated with malnutrition. Action potentials recorded from a blind pouch indicates that peristaltic waves propagating from oral to caudal collide against anti-peristaltic waves coming from the blind end. This anti-peristalsis seems to be responsible for the rebound retrograde transportation of the content.

Pathophysiology of the anti-peristaltic conduction will be discussed later.

In another series of experiments (Fig. 3) in which action potntials were recorded from the so-called "ganglion-free" ") circular muscle tube mounted on a glass rod of an appropriate diameter. The electrodes were placed at four different sites, each 0.5 or $1.0 \mathrm{~cm}$ apart on the intestine survived in a bath of Typrode solution. Despite the absence of the larger part of nervous element, peristaltic conduction was demonstrated in this preparation. But its direction tended to be divergent, being in iso- as well as in anti-peristaltic direction. Peristaltic conduction was directional in only $27.2 \%$ of the cases and non-directional in $72.7 \%$. In contrast, with preparation with both longitudinal and circular muscle layers and with Auerbach's plexus peristaltic conduction was directional in as much as $73.1 \%$ of the cases and non-directional in only $26.9 \%$. The velocity of peristalsis in the "glangion-free" ring muscle preparation was only $1.3 \mathrm{~cm}$ per second, and in the preparation with two muscle layers and nerve plexuses $2.3 \mathrm{~cm}$ per second. In general, the bi-directional peristalsis tended to occur more frequently in the "nerve-free" preparation and the velocity of peristaltic conduction was much slower. In an attempt to exclude the possible influence of the remaining nerve structures such as terminal reticulum, Cajal's cells etc., the circular muscle preparation was immersed in a bath of Tyrode solution containing $5 \times 10^{-7} \mathrm{~g} / \mathrm{ml}$ of tetrodotoxin. It is very interesting that under these circumstances in the total absence of nervous effects the peristaltic conduction was well maintained but not directional. 

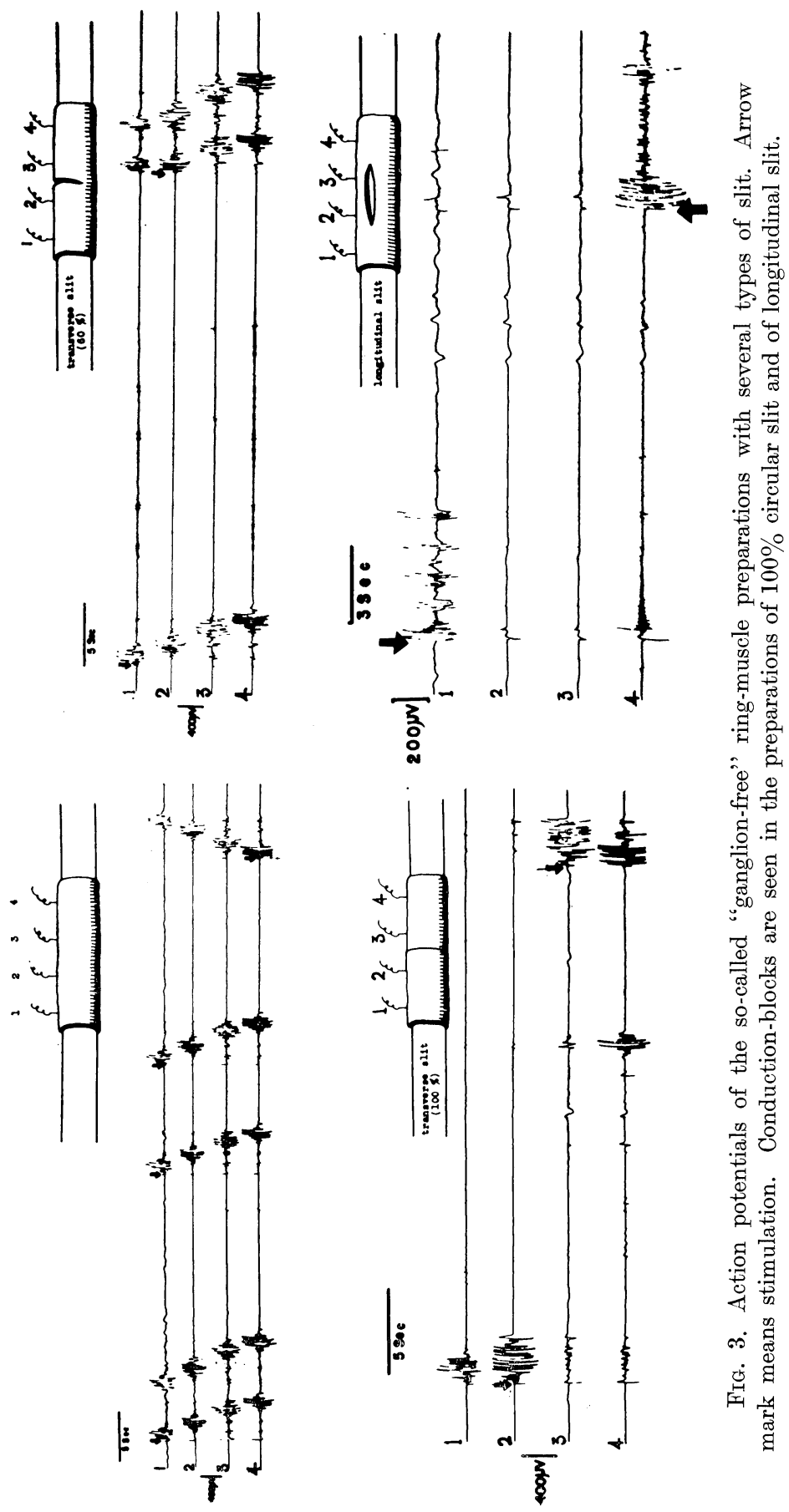
Complete or $100 \%$ circular section of the ring muscle tube failed to conduct the peristaltic excitation on the approximation of the both cut ends. When the circular section was limited to $60 \%$ of the whole circumference, complete transmission of the peristaltic excitation was shown to take place. Prosser ${ }^{6}$ ) reported that when a $100 \%$ circular section was made on the ring of a circular muscle preparation and the continuity was reestablished by pushing the both ends in contact, ephaptic transmission did take place over and cross the line of contact. The idea seems to satisfy the ardent desire of all surgeons. Our repeated experiments on similar ring muscle preparations, however, failed to demonstrate peristaltic transmission beyond the line of contact.

The pattern of peristaltic conduction following a side-to-side anastomosis in surgery simulates that of peristaltic conduction in the presence of a longitudinal slit in the so-called "ganglion-free" circular muscle tube. The peristalsis tended to be blocked at the two intermediately placed electrodes along the longitudinal slit. The longitudinal cleave in the intestinal wall is considered to give rise to the loss of tension and to lower the excitability, which, in turn, leads to the inability of the intestine to propel the content and the formation of a pouch-like dilatation. In view of these facts, the superiority of the end-to-end anastomosis to the side-toside anastomosis is suggested.

To clarify the most basic aspect of the smooth muscle function, ${ }^{7,8)}$ experiments were performed in the primordial digestive tracts of 40 human fetuses in 5 to 30 weeks. Figure 4 was made from a series of cinemicroscopic pictures of intestinal contraction at the 12th fetal week, showing two divergently propagating peristaltic rings. (The interval of each cinematographic frame is one second.) The

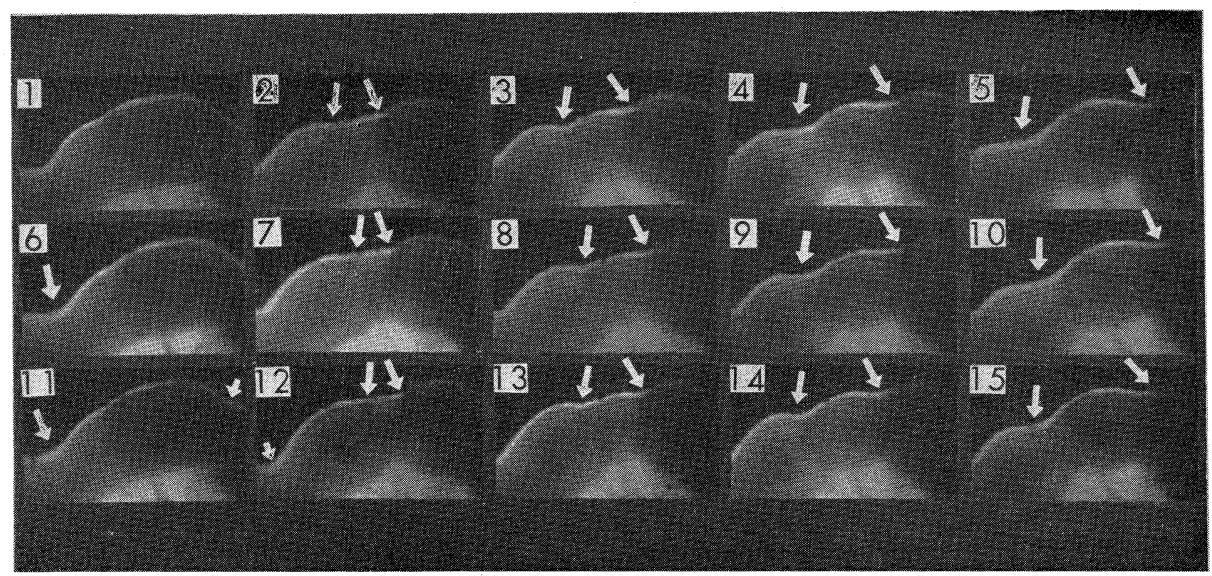

FIG. 4. Microscopic cinematography of peristaltic waves on the small intestinal wall in the early fetal stage (12 week).

The arrows show divergently propagating peristaltic rings. Interval of each cinematographic frame is one second. (Pflügers Arch. 279, 27, Abb. 2) 
contractions at an early fetal stage propagate orally as well as aborally in the type of peristalsis, very much like those of the so-called "ganglion-free" circular muscle tube.

Previous workers, including the author, ${ }^{7,8}$ have believed on the basis of their observations using the optical microscope that the longitudinal muscle layer and Auerbach's ganglion cells become demonstrable one week subsequent to the evolution of the circular muscle. However, our recent electronmicroscopic study has disclosed the presence of nerve structures in the intestinal wall of a six week old embryo. Figure 5 shows the primordial structure of the intestine with neuroblasts $(\mathrm{Nb})$, fibroblasts $(\mathrm{F})$ and axons $(\mathrm{Ax})$ with Schwann cells (S). This electronmicroscopic demonstration $\left.{ }^{9}\right)$ of the nerve structure in this primordial intestine where there is no discernible circular muscle, has presented an evidence to change our previous view. Prior to the 30th fetal week, peristalsis is not directional even in the presence of well stainable nerve structures. Therefore, it is reasonable to assume that the nerve cells play no active role in the regulation of the peristalitic direction prior to the 30th week of fetal life.

We have as yet no anatomical or physiological evidence to support the view that anti-peristalsis is suppressed through the regulatory control of fully developed myenteric plexuses.

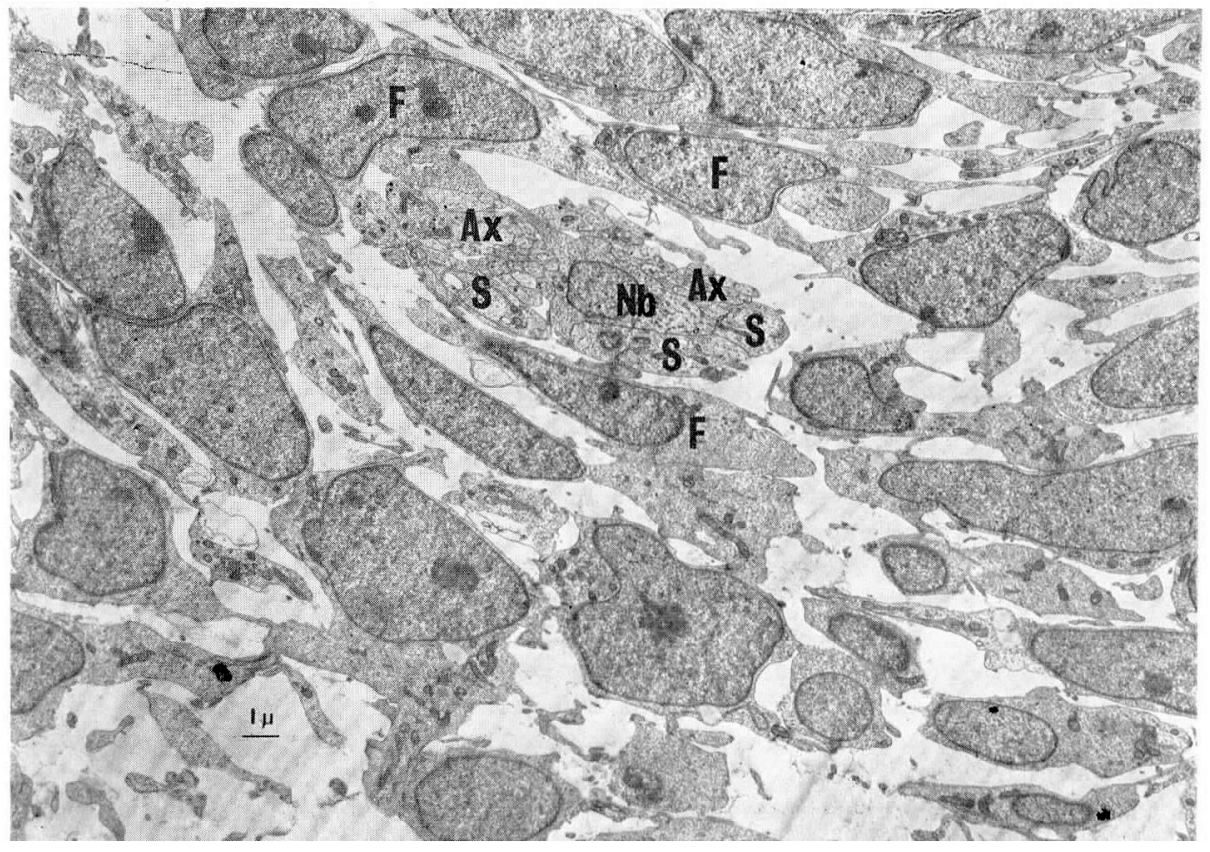


Our recent evidence has demonstrated the presence of markedly increased synaptic vesicles in the axon and its vicinity, especially at a later stage of fetal life. A more detailed morphological study of smooth muscle and nerve elements in the gut wall will be required for a better understanding of the function of synaptic vesicle and other microstructures related to the physiology of smooth muscle. The bi-directional conduction of peristalsis in the fetal intestine or in the "ganglion-free" ring muscle preparation may have been resulted from the immaturity or impaired function of the intramural nerve plexus. The most probable reason why antiperistalsis ceases to occur in the later stage of fetal life may be that immature ganglion cells begin to assume an active role in the determination of peristaltic direction. However, a possible aquisition of a "conditioning" of the digestive tract may be of some importance. The contents of the alimentary tract in a fetus are likely to be driven analward rather than oralward where the tract has a higher intraluminal pressure. Anti-peristalsis occurring during early fetal life would encounter a progressively higher resistance to the flow of the content, while the flow in the iso-peristaltic direction is increasingly facilitated by the hydrostatic downflow of the content. This may be called a "conditioning" of the digestive tract. The conduction of the peristaltic waves becomes increasingly uni-directional as the fetal growth continues. The end result of this process of conditioning would be the establishment of what we call the "Alvarez's gradient." 10 ) In the event of a situation where a pathological process forces the intestine to make the backflow of the content, the latent anti-peristaltic conductivity of the intestine becomes manifest to adapt the digestive tract to such a situation.

\section{References}

1. Takita, S.: Pathophysiology of anastomosis of the alimentary tract. Bulletin de la Societe Internationale de Chirurgie, 27, 456-463, 1968.

2. Fujioka, O.: Experimental studies on the healing process of the anastomosed intestine. Acta Medica, 29, 1193-1214, 1959, (in Japanese).

3. Inoue, T.: Experimental studies on the healing process of the esophageal anastomosis after total gastrectomy. Shikoku Acta med., 16, 1028-1044, 1960, (in Japanese).

4. Tsujimura, T.: Electrophysiological studies concerning the propagation of impluse in the operated intestine after end-to-end anastomosis. Jap. J. Smooth Muscle Res., 1, 30-41, 1965, (in Japanese).

5. Tsujimura, T.: Electrophysiological studies on the peristaltic propagation in the side-to-side anastomosed loops. Jap. J. Smooth Muscle Res., 3, 48-57, 1966, (in Japanese).

6. Prosser, C.L. and Sperelakis, N.: Transmission in ganglion-free circular muscle from the cat intestine. Amer. J. Physiol., 187, 536-545. 1956.

7. Takita, S.: On the direction of the peristaltic conduction of the intestine. Jap. Journ. Physiol., 7, 286-296. 1957.

8. Takita, S.: Über den Urpsrung der Peristaltik im Frühstadium des menschlichen Fetus. Pflügers Arch. ges. Physiol., 279, 26-35, 1964.

9. Kubozoe, T., Daikoku, S. and Takita, S.: Electronmicroscopic observations on Auerbach's plexus in a $12 \mathrm{~mm}$ human embyro. Journ. Neuro-Visceral Relations, 31, 291-307, 1969.

10. Alvarez, W.C,; The mechanics of the digestive tract. 2. ed., Paul B. Hoeber, Inc., 1928. 


\title{
The Function of Intestinal Slow Waves in Coordinating Mechanical Activity
}

\author{
ALEXANDER BORTOFF, M.D. \\ Department of Physiology, State University of New York, Syracuse, N.Y., U.S.A.
}

One of the most characteristic physiological properties of intestinal smooth muscle is its intrinsic rhythmical contractile activity, which, in the intact animal, tends to be propulsive. Rhythmical contractions are normally exhibited by both muscle layers, although they are not evident in circular muscle when completely separated from the longitudinal layer. It is the purpose of this communication to correlate the various patterns of intestinal motility with those of slow wave activity.

The relationship between slow waves and contractions in intestinal muscle is well documented, at least for longitudinal muscle. Slow waves represent periodic depolarizations of intestinal muscle, which, if of sufficient magnitude, result in spiking. This, in turn, triggers the contractile process. The rhythmical nature of intestinal motility is thus a function of the slow wave. Rhythmical contractions of circular muscle are likewise a result of slow wave activity. However, slow waves are actively generated only by longitudinal muscle and appear to be transmitted via interconnecting bridges to the circular layer where they spread electrotonically. Electrotonic spread in circular muscle is indicated first by the fact that monophasic slow waves can be recorded across the two muscle layers when intact, but not across either one separately; secondly, by the exponential decay of slow wave amplitude in circular muscle as a function of distance from the longitudinal layer; thirdly, by the virtually simultaneous time of slow wave onset in corresponding points of the two muscle layers; and finally, by the difference in configuration of slow waves recorded in volume from both muscle layers simultaneously. The slow wave recorded in volume from longitudinal muscle is generally triphasic with a large negative monophasic component, whereas that recorded from circular muscle is simply monophasic and positive. Hence, slow wave depolarization appears to be active in longitudinal muscle, and passive in circular. The simultaneous onset of slow waves in both muscle layers causes them to contract simultaneously, not $90^{\circ}$ out of phase as has been suggested.

The pattern of mechanical activity recorded from various points along the intestine also appears to be a function of slow wave activity. This applies to both the frequency gradient and the direction of propagation. We have recently 
studied the slow wave frequency gradient in several species of animals, using suction electrodes. It was found that in intact segments of intestine, both in vivo and in vitro, the frequency of slow waves decreases aborally in stepwise fashion, with variable lengths of intestine having the same frequency. Each frequency plateau is invariably separated from the next by an area of waxing and waning, the pattern of which can be reproduced by shorting together the input leads from electrodes placed in the two adjacent plateaus. The lengths of the plateaus, and consequently the positions of waxing and waning are not constant. When the intestine was cut into small segments, the slow wave frequency of each segment was found to decrease aborally in linear fashion. These phenomena could be explained on the assumption that intestinal smooth muscle behaves electrically as a series of loosely coupled oscillators having successively decreasing intrinsic frequencies. In a frequency plateau individual oscillators are effectively driven by the oscillator having the highest intrinsic frequency. At the end of the plateau, either the coupling between oscillators is inadequate (possibly due to a disruption of nexuses, such as can occur during mechanical activity), or the frequency of the driving oscillator is too high for the next oscillator to follow. The latter then assumes the role of pacemaker for the next frequency plateau. Waxing and waning appears to be an interference phenomenon resulting from the difference in slow wave frequencies of adjacent cell populations.

The two slow wave patterns, plateaus and waxing and waning, can be related to propagation. In a frequency plateau, slow wave propagation tends to be uniform. In a waxing and waning zone, on the other hand, propagation appears to be interrupted especially during the waning phase. In terms of the mechanical rise to spike potentials, propulsive mechanical activity would result. Because slow wave propagation is interrupted at the waxing and waning zone, the latter could serve as a mechanical stopcock, preventing mechanical activity from proceeding through the zone except for a brief time during the waxing period when the phase difference between slow waves in adjacent plateaus is minimal. Thus, although slow waves probably do not propagate from one plateau to the next, mechanical activity may do so to a limited extent. In general, however, propulsive mechanical activity would appear to be self-limiting; because of the apparent lability of the nexuses, the lengths of the plateaus would be shortened, with the number of waxing and waning areas ("stopcocks") increased in direct relation to the degree of mechanical activity. This may explain why "peristaltic rushes" rarely occur in the small intestine. 


\title{
The Electrical Activities of Guinea-pig Small Intestine
}

\author{
TAKESI HUKUHARA, M.D. \\ Department of Physiology, Okayama University Medical School, Okayama, \\ Japan
}

By utilizing the ultramicroelectrode technique combined with Trendelenburg's method the electrical activities were studied on the isolated guinea-pig jejunal loop.

This method enabled one to observe the course of electrical activities occurring when the excitability of the intestinal muscle was gradually increased as high as a critical level at which pulsations could be produced:

(1) With the rise of exictability of the intestinal muscle, the depolarization of the muscle cell membrane was gradually increased, being superimposed with minute oscillations or waves. And when the peak of the wave reached the firing level, the spike was produced (Fig. 1).
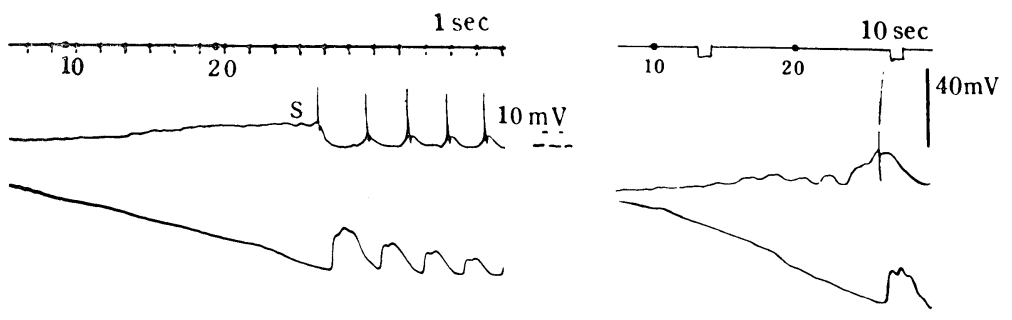

Fig. 1. Electrical activity detected intracellularly from an isolated guinea-pig jejunal loop.

Tracings from above downwards indicate time, intracellular recording of electrical activity of, and volume change of the loop. Dots seen on the uppermost tracing show the moments at which the intraluminal pressure is raised as high as the water column $\left(\mathrm{mmH}_{2} \mathrm{O}\right)$ indicated by numerals written undereneath.

(2) The spike contributed to the further development of the slow wave in a way described below: The spike was successively followed by the initial afterhyperpolarization (IAHP), the late after-depolarization (LDAP) and the delayed after-hyperpolarization (DAHP), the last named one soon turning to the phase of an increasing depolarization, prepotential (PP), until it culminated in producing a spike again (Fig. 2). 


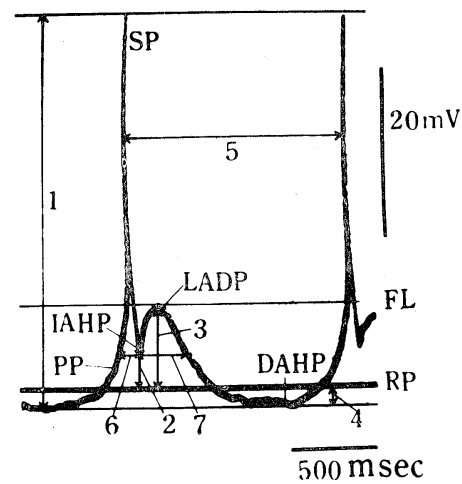

FIG. 2. A spike accompanied with slow wave (magnified to two times to show the details of the electrical event).

PP: Prepotential, FL: Firing level, SP: Spike, IAHP: Initial after-hyperpolarization, LADP: Late after-depolarization, DAHP: Delayed after-hyperpolarization RP: Resting membrane potential, I: Amplitude of spike potential, 2: Depth of IAHP, 3: Amplitude of LADP, 4: Amplitude of DAHP, 5: Time interval of spike potential, 6: Half duration of prepotential, 7: Half duration of LADP.

(3) If spikes were abolished by lowering the pressure, the waves became smaller in their amplitude and shorter in their period, until they were reduced to minute oscillations. In short, the fluctuations of depolarization, ranging from slow waves to minute oscillations, may essentially be of the same nature.

(4) The electical activities were enhanced by raising the intraluminal pressure of, or applying acetylcholine to the intetinal loop, whereas they were suppressed by lowering the pressue of, or applying nicotine as well as atropine to the loop: The pressure-raising accelerated the rise of $\mathrm{PP}$ and enhanced the development of LADP (Fig. 3), contributing the frequent elicitation of spikes (Fig. 4).
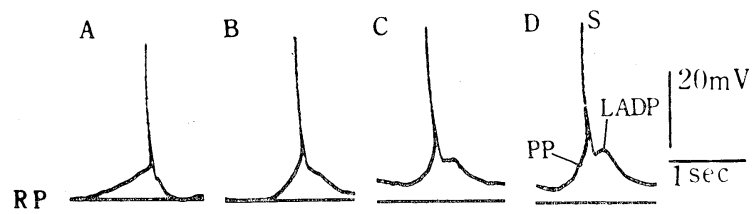

FIG. 3. Tracings from A to D indicate aspects of electrical events detected intracellularly under the intraluminal pressures, 10, 20, 30 and $40 \mathrm{mmH}_{2} \mathrm{O}$, respectively.

PP: Prepotential, S. Spike, LADP: Late after-depolarization, RP: Resting membrane potential.

(5) Since the pressure-raising can eventually be regarded as a factor which enhances the release of acetylcholine (Hukuhara and Fukuda')), the facts described above thus suggest that acetylcholine plays an important role in initiating the fluctuations in the depolarization. It is presumed that the greater the amount of acetylcholine is released from its source, i.e., intramural nervous 


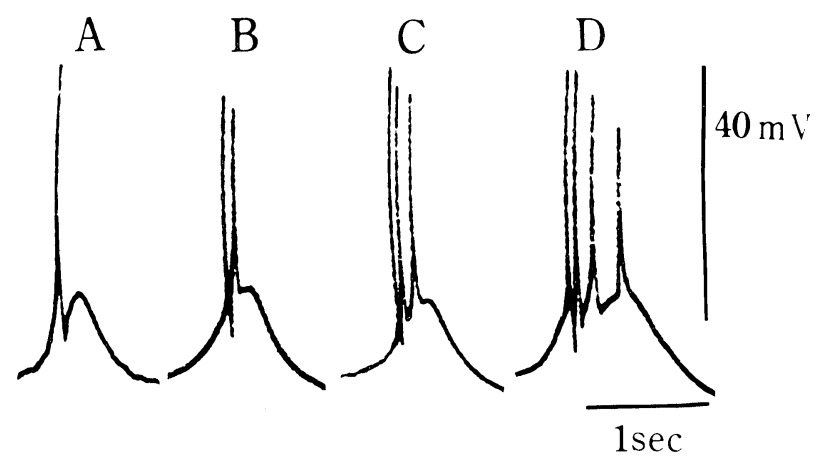

FIG. 4. The influence of the intraluminal pressure upon the electrical activities.

The pressure was raised stepwise from A to $\mathrm{D}$ with increase of $10 \mathrm{mmH}_{2} \mathrm{O}$ until it reached $40 \mathrm{mmH}_{2} \mathrm{O}$. It was noted that the higher the pressure was, the more the spikes were produced.

elements, the larger is the amplitude of the fluctuations, and that the greater the amplutide of the fluctuation is, the higher is the excitability of the intestinal muscle. In addition, Hukuhara et al. ${ }^{2}$ showed that acetylcholine released from intramural nervous elements was responsible for maintaining the rhythmic contractions of the small intestine.

\section{References}

1. Hukuhara, T. and Fukuda, H. : The motility of the isolated guinea-pig small intestine. Jap. J. Physiol. 15: 125-139, 1965.

2. Hukuhara, T., Nakayama, S. and Fukuda, H.: On the problem whether the intestinal motility of a neurogenic or myogenic nature. Jap. J. Physiol. 15: 5I5-522, 1965.

Notice: The contents presented here has in detail been reported in Jap. J. Physiol. 18: 71-86, 1968, under the title, "The electrical activity of guinea-pig small intestine with special reference to the slow wave". 


\title{
The Electric and Mechanical Activity of the Gastroduodenal Junction
}

\author{
PAUL BASS, Ph.D. \\ Parke, Davis \& Company Research Laboratories, Ann Arbor, Michigan, U.S.A.
}

Electric and contractile studies have been performed on the antral-duodenal area of chronic unanesthetized dogs. Electric activity was recorded by implanted extracellular electrodes; muscle contractility (frequency and force) was recorded by extraluminal force transducers. The basic electric rhythm (BER) of the antrum has a mean frequency of 4.4 cycles/min. and is occasionally associated with spike potentials. The frequency of the BER in the duodenal bulb is the same as the rest of the duodenum (18.5 cycles/min.), but its voltage is less and its form more irregular. Bursts of spike potentials in the bulb, when present, tend to occur at the same frequency as the BER, but the temporal association between the two electric phenomena is not as constant as in the remainder of the duodenum. The hypomuscular segment between the antrum and duodenum acts as an electric insulator and the electric recordings from this area are minimal or absent.

Relationships of the two electric phenomena between the two organs were studied in both the fasted and postprandial states (following $200 \mathrm{gm}$. of canned $\operatorname{dog}$ food). In the fasted state, there is no correlation between the occurrence of the BER of the antrum and duodenum; the relationship of bursts of spike potentials between the two organs have not been studied. After feeding, the frequencies of the BER of the two organs still appear unrelated, but occurrence of spike potentials of the duodenum are correlated with the occurrence of antral BER and spike potentials. This latter interrelationship suggests that food ejected into the duodenum might ke responsbile for the initiation of the spikes in the duodenum.

It has keen demonstrated that the BER in the two organs is omnipresent and not necescarily accompanied by smooth muscle contractions. In contrast, spike potentials, when present, represent the electrical phenomena that preceles circular muscle contractions. The following results were obtained from recording smooth muscle activity. The accompanying electric phenomena were not studiex.

The relationship between antral and duodenal muscle contractions is shown in the figure (top). The influence of three test meals of known composition were evaluated for their effect on stomach emptying (Hunt's method) and on antral and duodenal contractility. The gastric emptying of a 200 milliosmol test meal of trisodium citrate was significantly more rapid than equal osmolar meals of glucose 
or trisodium citrate with $10 \mathrm{mN}$ oleic acid (citrate-fat). The contractile activity of the gastric antrum was higher during emptying of the citrate test meal as compared to the other two test meals. In contrast, the contractile activity of the duodenum was signfiicantly higher for the citrate-fat and glucose test meals as compared with the citrate test meal. This data indicates that gastric emptying is regulated in part by the relative contractile force of the antrum and duodenum. The exponential emptying rate of the stomach is directly related to the force of the antrum (as seen with the citrate test meal). The duodenal contractile activity also influences gastric emptying (as seen with the citrate-fat test meal). The greater rate of emptying occurs when there is relatively high antral and low duodenal activity; conversely, the slowest emptying occurs when there is relatively low antral and high duodenal activity.
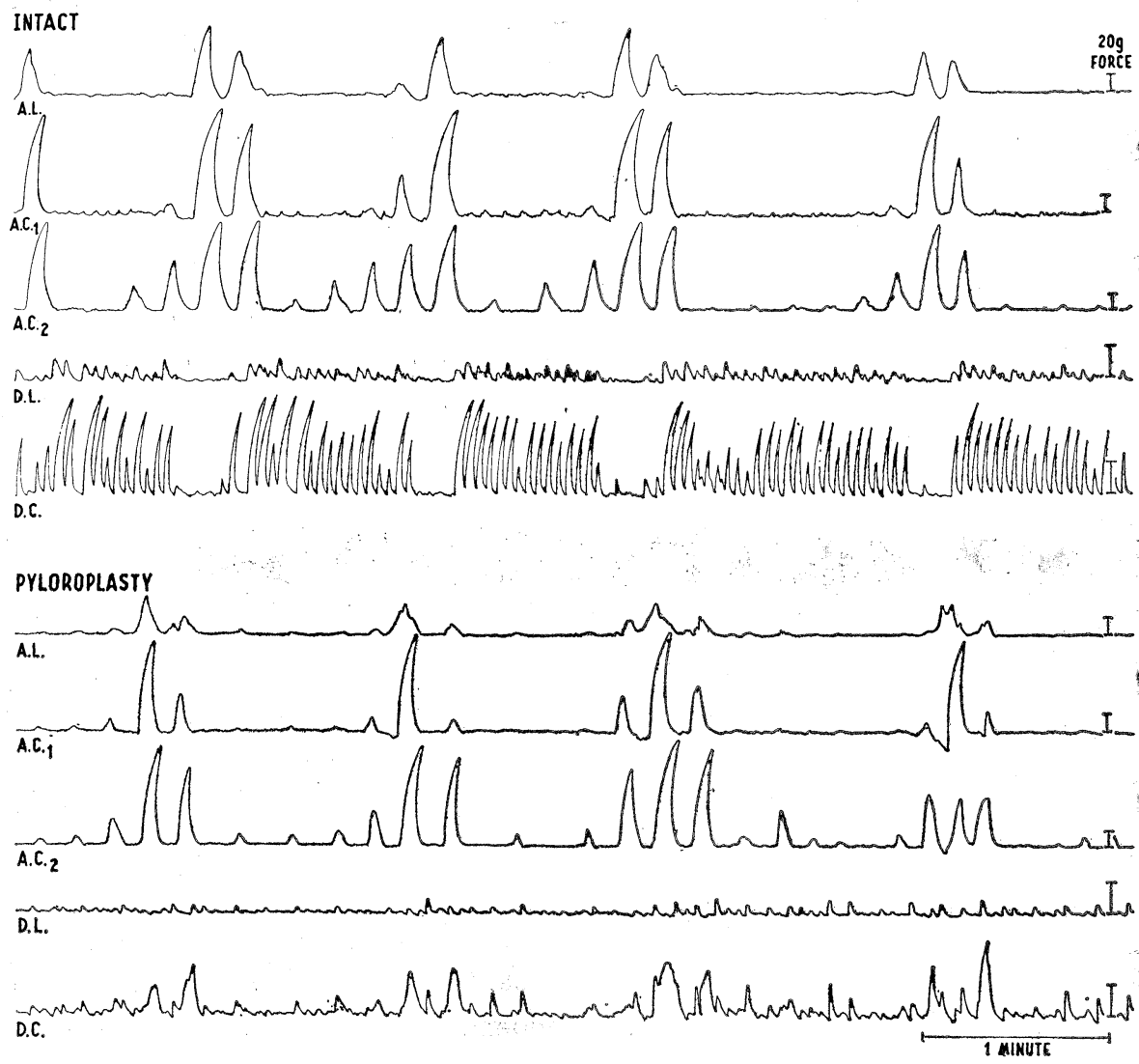

Fig. 1: Interdigestive contractile activity of the canine antrum and duodenum, intact (top) and after pyloroplasty (bottom). The units were orientated to record the contractility in the following axes: antral longitudinal (AL), proximal $\left(\mathrm{AC}_{1}\right)$ and distal $\left(\mathrm{AC}_{2}\right)$ antral circular, and duodenal circular (DC) and longitudinal (DL). Note that the larger antral contractions in the intact state are associated with reflex inhibition of the duodenum. Following pyloroplasty this was abolished and the two areas contracted simultaneously. 
The effects of a Finney pyloroplasty on antral-duodenal contractile activity and gastric emptying were also studied. The surgical alteration abolished the normal antral contraction-duodenal inhibition cycle and the two organs contracted simultaneously (Fig., bottom). The pyloroplasty also tends to decrease contractile force in the antrum and duodenum. The addition of vagotomy further decreases the force in the distal antrum but does not alter the simultaneous contractions induced by the pyloroplasty.

Gastric emptying was evaluated using three test meals in dogs in the intact state, following Finney pyloroplasty, and with the addition of vagotomy. The Finney pyloroplasty alone tended to further delay the gastric emptying rate of a citrate-fat test meal. The addition of vagotomy enhanced the rate of gastric emptying toward the intact condition. The emptying characteristics of the citrate or glucose test meals were not signfiicantly altered by the pyloroplasty. The level of gastric secretion increases after performing the pyloroplasty. The pyloroplasty institutes an abnormal opening between the antrum and duodenum which causes an alteration of the intergraded antral-duodenal contractile relationship. This change impaired gastric emptying of a citrate-fat test meal to a variable degree suggesting that abnormal stomachs alter the normal antral-duodenal relationship and lead to a contractile pattern that is obstructive to emptying. 
Jap. J. Smooth Muscle Res., 6, 97 98, 1970

\title{
The Electrical Activity of the Stomach and Upper Intestine as Determinant of Mechanical Activity
}

\author{
E. E. DANIEL, Ph.D. AND J. IRWIN, M.D. \\ Department of Pharmacology and Department of Surgery, The University of \\ Alberta, Edmonton, Alberta, Canada
}

In each of the distal stomach and the proximal small intestine, two characteristic kinds of electrical activity are found. One designated control activity, occurs periodically, is propagated distally and is not necessarily related to contraction.

In the dog stomach, control activity consists of a 4 to 5 per minute spike-like depolarization (initial potential; also called slow wave, pacesetter potential [PSP] or BER) of the transmembrane potential (triphasic volume-conducted response with monopolar recording); in the duodenum, control activity consists of a 17 to 20 per minute slow depolarization (slow wave, BER or PSP) of the transmembrane potential (sometimes triphasic volume conducted response; sometimes diphasic). Control activity seems to originate in and spread (or be coupled) distally via longitudinal muscle cells. Transection of the stomach or intestine leads to disruption of distal spread or coupling of control activity, distal regions taking up a slower frequency of discharge. Control activity modifies the nature of the response of muscle to chemicals injected locally or released by local and other reflexes. In the stomach, small doses of acetylcholine injected intra-arterially cause response electrical activity and contraction if injected within 3 to 5 seconds after passage of next initial potential, the same dose may have no effect or may initiate a premature initial potential with or without subsequent response activity. Such premature initial potentials are often propagated antidromically; and, if so, they interfere with the subsequent normally propagate initial potentials and may alter the rhythm of proximal areas for several cycles. Catecholamines in the smallest effective doses inhibit response electrical activity and contractions without effecting control electrical activity. Higher doses, given intra-arterially, inhibit as well the propagation or coupling of control activity, whether this be normal propagation from a "pacemaker" or antidromic propagation from a premature initial potential induced by acetylcholine. Distal areas can take up their own frequency of control activity after decoupling by catecholamines. Furthermore, distal inhibition of an initial potential by intra-arterial catecholamines can temporarily alter the subsequent rhythm of proximal initial potentials. Under certain circumstances large doses of 
catecholamines or atropine initiate a high frequency discharge of initial potentials from the pyloric portion of the stomach which propagates antidromically. These findings and others are interpreted to suggest that control activity in the stomach arises from an inherent rhythmical discharge of longitudinal muscle cells, with proximal regions having the higher inherent frequency which by means of an electrotonic forward coupling process drives distal regions. However, backward coupling also can occur. Acetylcholine can initiate control activity locally and catecholamines can decouple control activity spreading proximally or distally. In the duodenum, control activity can also be decoupled by larger intra-arterial doses of catecholamines, but acetylcholine cannot readily be shown to initiate slow waves, and backward coupling or propagation of slow waves has not been proved. Whether the initiation and coupling of control activity occurs by similar mechanisms in the duodenum and stomach is uncertain. Evidence will be presented that nervous reflexes can operate by effecting control activity as well as response activity.

Response electrical activity is associated with contraction and consists of spikes in the intestine or prolonged secondary depolarization with or without spikes in the stomach. It is not propagated effectively in either stomach or duodenum. It is initiated by acetylcholine and inhibited by catecholamines, and normally occurs at a time determined by the control activity. In the stomach, and possibly in the duodenum, response electrical activity does not occur without the immediately previous occurrence of control electrical activity. The possible nature of the connection between control and response electrical activity will be discussed. 
Jap. J. Smooth Muscle Res., 6, 99 101, 1970

\title{
The Control of Gastric Motility by the Gastric Pacesetter Potential
}

\author{
KEITH A. KELLY, M.D. ANd CHARLES F. CODE, M.D., Ph.D. \\ Mayo Clinic and Mayo Foundation: Department of Surgery (Dr. Kelly) and of \\ Physiology (Dr. Code), Rochester, Minnesota, U.S.A.
}

The objective of our experiments was to test two hypotheses: (1) that the gastric pacesetter potential or slow wave, as it spreads through the gastric wall, controls and coordinates gastric motor activity; and (2) that stimuli alter gastric motions through their effects on the gastric pacesetter potential.

In each of 15 dogs, eight silver-silver chloride electrodes were implanted at equal intervals (approximately $3 \mathrm{~cm}$ ) from the fundus to the pylorus on the anterior serosal surface of the stomach. Beginning 2 weeks after the operation, monopolar, electric recordings were made during fasting, after insulin-induced hypoglycemia, and after gastric instillations of water and of cottonsecd oil. Gastric cinefluorography was also sometimes performed. After control observations had been made for 2 to 4 months, five of the animals underwent transthoracic vagotomy and were restudied for an additional 3 months.

A cyclic, recurring, triphasic fluctuation in potential (the pacesetter potential, PP) was always present in the caudad two thirds of the corpus and antrum of the stomach during fasting. ${ }^{1)}$ It originated in the orad corpus. The mean frequency of the cycles $(5.4 \mathrm{cpm})$ was the same in the corpus and antrum, but its velocity increased as the PP was propagated aborally from the orad corpus to the pylorus. The velocity of propagation in the corpus was about $0.4 \mathrm{~cm} / \mathrm{sec}$ and in the antrum about $1.4 \mathrm{~cm} / \mathrm{sec}$. The wavelength or cycle length of the PP (wavelength=velocity/ frequency) was shorter in the corpus $(4.0 \mathrm{~cm})$ than in the antrum $(15.0 \mathrm{~cm})$.

Action potentials that indicated contractions always occurred just after the triphasic fluctuation of the cycle and were associated with about one fourth of the antral PPs in the fasting state.

A narrow band of contraction moving slowing aborally was found on cinefluorography in the gastric corpus where the PP cycle length was short and the $\mathrm{PP}$ velocity was slow. In contrast, the wide, nearly simultaneous contraction seen in the terminal antrum was associated with the longer wavelength and faster velocity of the PP in this region. The length of the segment of the PP cycle that can support action potentials, therefore, must lengthen as the cycle length extends.

Insulin hypoglycemia had little effect on the frequency of the PP, but decreased the time required for propagation of the PP between adjacent corporal 
electrodes from 8.0 to 5.7 seconds, thereby increasing the corporal PP cycle length from 4.0 to $5.5 \mathrm{~cm}$. Action potentials were associated with $19 \%$ of the antral PP before insulin and $96 \%$ at the peak of the responses. Gastric contractions, therefore, were more frequent after insulin administration, and when they occurred the width of the band of contraction was probably broader, owing to the longer PP wavelength.

Gastric instillation of water decreassed the PP frequency from 5.4 to $4.9 \mathrm{cpm}$ and decreased the time required for propagation of the antral PP from 2.2 to 2.0 seconds, thereby increasing the antral wavelength from 15 to $18 \mathrm{~cm}$. The incidence of antral action potentials also increased from $14 \%$ before water instillation to $90 \%$ at the peak of the response after water instillation. Thus, water instillation, like insulin administration, resulted in broader as well as more frequent gastric contractions.

Fat instillation had no consistent effect on the frequency of the PP but lengthened the time required for corporal propagation from 6.6 to 8.3 seconds, thereby shortening the corporal cycle length from 5.0 to $3.7 \mathrm{~cm}$. The incidence of antral action potentials was also decreased from $54 \%$ before oil instillation to $5 \%$ after it. Fewer and narrower gastric contractions thus ensued.

Vagotomy disorganized the normal orderly pattern of gastric electric activity for 1 to 30 days. $^{2)}$ During this period, PPs arose at multiple sites and exhibited various amplitudes, frequencies, configurations, and conduction velocities. Coordinated, propulsive, peristaltic activity was lost in the presence of these disorganized rhythms.

Restoration of an orderly electric pattern occurred later, and then the frequency of the PP was similar to that before vagotomy, but propagation time was lengthened in the corpus from 7.9 seconds before vagotomy to 9.2 seconds after it. The cycle length, therefore, was shortened in the corpus from $4.2 \mathrm{~cm}$ before vagotomy to $3.6 \mathrm{~cm}$ after it and in the antrum from $15.2 \mathrm{~cm}$ before to $11.7 \mathrm{~cm}$ after vagotomy. Vagotomy also decreased the incidence of antral action potentials in two of the five dogs. Thus, narrower and, in some animals, fewer gastric contractions were found after vagotomy in the fasting state.

In addition, after vagotomy, insulin no longer increased the velocity of propagation of the PP nor the incidence of action potentials associated with it. The influence of water instillation on the PP pattern was unaltered by vagotomy, but oil instillation no longer decreased the incidence of antral action potentials after vagotomy.

These experiments support our original hypotheses. The PP coordinated gastric motions, and stimuli altered gastric motility by modifying the pattern of the PP in the gastric wall. Insulin-induced hypoglycemia and water instillation augmented gastric motility by increasing the cycle length of the PP and the incidence of action potentials. Fat instillation and vagotomy decreased gastric motions by 
shortening the PP cycle length and decreasing the incidence of action potentials.

A longer cycle length is associated with a broader or wider band of contraction during peristalsis.

This investigation was supported in part by Research Grant AM-2015 from the National Institutes of Health, Public Health Service.

\section{References}

1. Kelly, K.A., Code, C.F., and Elveback, L.R.: Patterns of canine gastric electrical activity. Amer. J. Physiol. 217, 461-470, 1969.

2. Kelly, K.A. and Code, C.F.: Effect of transthoracic vagotomy on canine gastric electrical activity. Gastroenterology, 57, 51-58, 1969. 


\section{The Frequency, Velocity and Cycle Length of the Pacesetter Potential (PP)}

JOSEPH H. SZURSZEWSKI, Ph.D. AND CHARLES F. CODE, M.D., Ph.D. Section of Physiology, Mayo Foundation, Rochester, Minnesota, U.S.A.

The purpose of this study was to define the caudad gradient of the PP in a number of species, to elucidate those factors which produce the gradient and to determine in dogs the velocity and wave length (cycle length) of the PP at multiple sites of the small bowel.

All electrical data were obtained from multipe unipolar and bipolar platinum electrodes previously sewn to the serosal surface of the small bowel. All recordings were made when the animals were healthy and conscious.

The mean frequency of the PP in the duodenum, mid-portion of the small bowel and the terminal ileum of unanesthetized dogs was 19.0, 16.5 and 14.2 waves/minute respectively. Thus, the ileal frequency was $27 \%$ less than the duodenal frequency. This value likely has signfiicance for the per cent reduction of the ileal frequency from that of the duodenum which we have found in man, cat, rabbit, rat and sheep is very similar, being $30 \%, 20 \%, 28 \%, 25 \%$ and $30 \%$, respectively with a mean of $27 \%$.

Others have identified the site of origin of the PP in the dog to be at or near the entrance of the common bile duct. Our studies have shown that the first $20 \%$ of the small bowel had an identical frequency. Beyond this initial plateau, the frequency decreases gradually (linearly in some instances and non-linearly in other instances) as the pattern of the PP becomes more variable.

Reductions in the PP frequency occur during spontaneous spike activity of several minutes duration and during prolonged spike activity within one PP cycle. Pharmacological stimuli which increase the incidence of spike potentials (urecholine, quinidine sulphate) also decrease the PP frequency, whereas a stimulus which reduces the incidence of spike potentials (epinephrine) increases the PP frequency. For exmaple, in 19 tests, the mean PP frequency in the duodenum was $19.1+$ $0.2 /$ min., in the jejunum $16.3+0.3 / \mathrm{min}$. and in the ileum $14.1+0.3 / \mathrm{min}$., whereas after an injection of urecholine $(100 \mu \mathrm{g} / \mathrm{kg}$. s.c.) the PP at these sites was decreased $1.3 / \mathrm{min}$., $0.8 / \mathrm{min}$. and $0.6 / \mathrm{min}$., respectively $(\mathrm{p}<0.01)$. In 18 other tests, intravenous infusion of epinephrine for 60 minutes $(2 \mu \mathrm{g} / \mathrm{kg} . / \mathrm{min}$.) after atropinization $(0.1 \mathrm{mg} . / \mathrm{kg}$. s.c. or i.v.) increased the duodenal and jejunal PP frequency by 1.2 waves $/ \mathrm{min}$. $(\mathrm{p}<0.01)$ and lengthened the initial frequency plateau from $64.6+5.4 \mathrm{~cm}$. to $9.55+5.4 \mathrm{~cm}$. beyond the pylorus. 
Reduction of the PP frequency also occurs during waxing and waning of the slow wave amplitude and waxing and waning is often associated with the occurrence of overlapping rhythms. This was observed most often in the upper small bowel during rapid duodenal frequencies. During periods of slower duodenal frequencies, waxing and waning was less frequent and the length of the initial plateau was longer.

Mechanical correlates have been recorded. Dropped or lost contractions were observed in dogs years ago by I.B. Smith in our laboratory while recording spontaneous rhythmic motor activity and when overlapping of two different motor rhythms occurred at a point of recording.

In dogs with a transected-reanastomosed duodenum, the PP frequency gradient was abolished below the transection and never restored. The PP frequency immediately caudad to the cut was similar to that in the terminal ileum. Reduction in the PP frequency during periods of spontaneous spike activity was not observed and the occurrence of periods of waxing and waning was less frequent.

Douglas, years ago, noted similar reductions in the frequency of rhythmic motor activity after bowel transections.

These data suggest that the PP frequency gradient exists because of the rapid duodenal frequency. Caudaded to the duodenal pacemaker and the initial PP frequency plateau, populations of smooth muscle cells (segments of bowel) cannot accept every PP because the mean frequency at which they can be driven is less than the PP frequency of the duodenum. We propose that the waxing and waning of the PP amplitude and the overlapping rhythms is probably an expression of this phenomena. The reduction of the PP frequency during episodes of spike activity indicate that action potentials, under certain circumstances, interfere with the generation of a $\mathrm{PP}$ or with its propagation and this phonomena contributes to the loss of PP cycles and so to the gradient of PP frequency.

The velocity of the PP also displays a caudadward decreasing gradient. In the duodenum of unanesthetized dogs, velocity varies from 10 to $20 \mathrm{~cm}$. $/ \mathrm{sec}$. It slows to 5 to $10 \mathrm{~cm}$. $/$ sec. in the mid-portion of the bowel and to 1 to $3 \mathrm{~cm}$. $/ \mathrm{sec}$. in the ileum. If the velocity and period of the PP is known for the same segment of bowel, then the spread of a single cycle of the PP in centimeters can be defined by the formula: wave or cycle length=velocity $(\mathrm{cm} . / \mathrm{sec}$.$) \times duration (sec.).$ Current estimates in the dog suggest a cycle length of 35 to $45 \mathrm{~cm}$. in the duodenum, 15 to $25 \mathrm{~cm}$. in the jejunum and 2 to $5 \mathrm{~cm}$. in the ileum. To test this hypothesis, 25 unipolar electrodes were sewn at $2 \mathrm{~cm}$. intervals to the duodenum and proximal jejumnum. The calculated cycle length was obtained by determining the velocity and period of one wave and optically expanding this some wave to its calculated length. The voltage of 8 electrode sites was calculated for a fixed point in time and compared to that of the optically expanded wave. In all instances, there was good agreement between the calculated cycle length and the actual wave as it was 
spread out over the bowel. The concept that the PP is actually spread out to the calculated length in the other portions of the bowel requires testing. If it is, then the length of a physiological segment would also display a caudadward decreasing gradient. One of the important implications of this concept is that if the electrical and mechanical activity could be frozen for an instant, then the maximum number of rhythmic contractions over the whole length of the small bowel is limited by the number of PPs which simultaneously exist over the total length of small bewel.

Although a cycle of the PP can cover distances as long as $45 \mathrm{~cm}$., its most excitable zone (the location of the occurrence of spike potentials) is much less. Using 0.5 sec. as an estimate of the period of maximum excitability, the excitable zone of the intestine would be 5 to $10 \mathrm{~cm}$. long in the duodenum and upper jejumum, 1.5 to $3.0 \mathrm{~cm}$. in the mid-portion of the bowel and less than $1 \mathrm{~cm}$. in the terminal ileum. Measurements obtained with balloons in tandem of the length of bowel supporting one contraction during rhythmic segmentation ranged from 5 to $15 \mathrm{~cm}$. in the upper jejunum to 1 to $3 \mathrm{~cm}$. in the mid-small bowel. We propose that the wave or cycle length of the PP defines a physiological segment of the small bowel and that it regulates the motor activity within this segment by limiting the occurrence of spike activity to a restricted portion of the cycle length and so to a restricted portion of the intestinal segment. 


\title{
Some Electrical Events in Circular Muscle of Cat Colon
}

\author{
JAMES CHRISTENSEN, M.D. AND GORDON F. LUND, M.D. \\ University of Iowa College of Medicine, Iowa, U.S.A.
}

\begin{abstract}
We studied electrical events in vitro in cat colonic muscle with an external glasspore electrode and with intracellular glass microelectrodes. We used the isolated whole colon, isolated tubular segments of the colon, and isolated strips of colon muscle. Tension was recorded at the same time in the isolated muscle strips. In the outer longitudinal muscle layer, both types of electrodes detected spike bursts occurring only with contractions; microelectrodes showed small prepotentials before the spike bursts. In the circular layer, both types of electrodes detected constant spontaneous slow waves. Intracellular microelectrodes showed them to be periodic uniform membrane depolarization of $11.8+(\mathrm{SD}) 6.5 \mathrm{mV}$. The rapid depolarization led to a plateau with slower repolarization. Amplitude of the slow waves was directly proportional to the membrane potential between slow waves. Duration of slow waves varied from three to nineteen seconds, and the duration was directly proportional to the length of the accompanying contractions in isolated strips. The glass-pore electrode recorded the depolarization as a rapid biphasic spike, the plateau as an isoelectric pause, and depolarization as a slower biphasic signal. In the proximal colon, slow waves were rather irregular and slow, one to four per minute; in the distal colon they were very regular at five to seven per minute. Slow waves were propagated in the direction of the long axes of the cells of the circular layer at $1.6+(\mathrm{SD}) 0.43 \mathrm{~cm} . / \mathrm{sec}$. Caudad propagation, transversely across the cells, also occurred for variable distances at variable slow rates, one to five $\mathrm{mm}$./sec. These slow waves seem to be similar to the spontaneous slow waves of small bowel and stomach. They probably have an important role in governing the movements of the colon.
\end{abstract}


Jap. J. Smooth Muscle Res., 6, 109 111, 1970

\title{
Calcium Movement and Action Potential in Smooth Muscle
}

\author{
TAIZO SUZUKI, M.D. AND JUNICHIRO NAGASAWA, M.D. \\ Department of Applied Physiology, Tohoku University School of Medicine, \\ Sendai, Japan
}

It is well known that the generation of action potential in the smooth muscle is not strictly dependent on the $\mathrm{Na}$ concentration of the external fluid, and tetrodotoxin (TTX), which is known to be a specific Na spike inhibitor in many excitable tissues, can not stop the generation of action potential in the smooth muscle.

On the other hand, Mn ion, which is known to be an inhibitor of the Ca spike in the crustacean and barnacle muscles, also inhibit the action potential of the smooth muscle of guinea-pig taenia coli. These evidences strongly suggest that the action potential of the taenia coli is generated by the influx of $\mathrm{Ca}$ ion. The direct way to prove this, is to show the increment of the intracellular $\mathrm{Ca}$ as the result of the evolution of action potential. But there are many difficulties in conducting such experiments, the major one is the presence of spontaneous action potentials in this tissue. Their irregular occurance made it almost impossible to get the, so called, resting flux of the taenia coli.

As mentioned above, application of low concentration of $\mathrm{Mn}$ ion could stop the generation of action potential of taenia coli completely, without causing any appreciable change in the resting potential, and this Mn ion effect is reversible as long as its concentation is low and the application time is not too long. So it seems possible to compare the resting $\mathrm{Ca}$ influx and the extra entry of Ca ion accompanying the generation of action potential using $\mathrm{Mn}$ ion as the tool, if it could be assumed that $\mathrm{Mn}$ ion in low concentration exerts no deleterious influences on the membrane characteristics and the metabolism of the smooth muscle cells.

Experimental method: As is well known, wash-out curve of ${ }^{45} \mathrm{Ca}$ loaded in taenia coli composed roughly the sum of two exponentials. The fast phase is mainly concerns the loss of tracer presenting in the extracellular space. To avoid the influence of this fast phase, all the efflux experiments were carried out after 30 minutes preliminary washing in inactive solution. And the wash-out curve of the present experiments were considered to express mainly the movement of intracellular Ca. In some instances, more slowly decline third component was observed after 90 minutes of washing. But its exact evaluation was impossible and was neglected in this experiments. 
From the extrapolation of the linear portion of the second phase to zeroline axis, Ca content taken up during the load period of 15 minutes was calculated (Fig. 1). The residual ${ }^{45} \mathrm{Ca}$ at the end of experiment was counted exactly and uptake of ${ }^{45} \mathrm{Ca}$ was calculated from the mean half-time obtained initially.

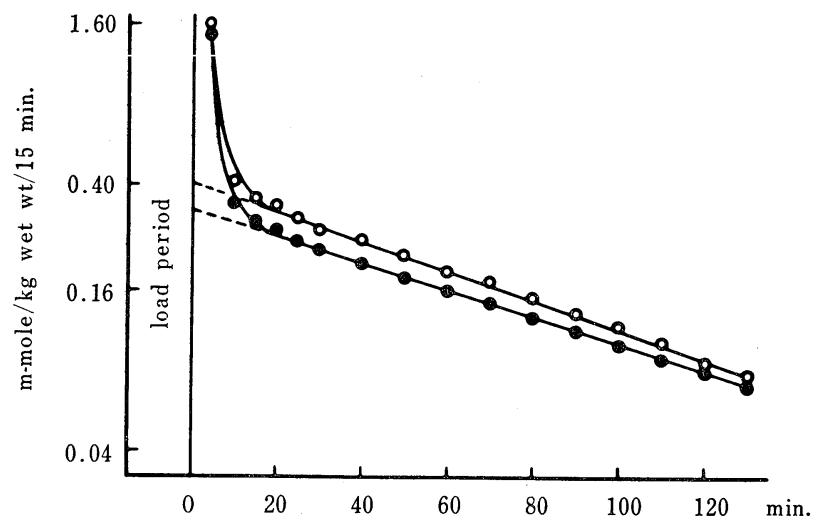

FIG. 1. Schematic representation illustrating the method of calculation of uptake of Ca during load period of $15 \mathrm{~min}$. Two preparations from the same guinea-pig were loaded in separate loading solutions, one is control loading solution (open circles), the other in the loading solution containing $1 \mathrm{mM} \mathrm{MnCl}_{2}$ besides normal ionic composition (solid circles). Extrapolation of the linear portion to O-time gave the Ca content taken up by the muscle during load period.

In this experiment Ca uptake in normal Krebs solution and in Krebs solution containing $1 \mathrm{mM} \mathrm{MnCl}_{2}$ was estimated and $\mathrm{Ca}$ influx accompanying evolution of action potential was tentatively calculated as the difference between them.

Results and Comment: Mn ion had no significant effect on the efflux of ${ }^{45} \mathrm{Ca}$ in the concentration range between 1.0 to $10 \mathrm{mM}$ (Fig. 2). The rate coefficient for the $\mathrm{Ca}$ loss seemed sligtly decreased directly after the application of $1 \mathrm{mM} \mathrm{MnCl}_{2}$, but this was proved to be not significant by the statistical analysis, owing to the large error. In the presence of $1 \mathrm{mM} \mathrm{MnCl}_{2}$, adrenaline (up to $2.5 \times 10^{-5} \mathrm{~g} / \mathrm{ml}$ ) and tetrodotoxin (up to $2.5 \times 10^{-3} \mathrm{~g} / \mathrm{ml}$ ) had no significant effect on ${ }^{45} \mathrm{Ca}$ efflux (Fig.3). But acetylcholine (up to $2.5 \times 10^{-3} \mathrm{~g} / \mathrm{ml}$ ) was found to increase the ${ }^{45} \mathrm{Ca}$ efflux coincidently with the tension development.

Using the values of Goodford et al., it is assumed that the surface area of smooth muscle cell membrane of $1 \mathrm{~kg}$ wet wt. of taenia coli is $3.3 \times 10 \mathrm{~cm}^{2}$, the tissue specific gravity is 1.1 and the muscle cell is uniform cylinder of $6 \mu$ in diamter. From this, total Ca influx in normal Krebs solution was calculated to be $0.135 \mathrm{p}$-mole $/ \mathrm{cm}^{2}$. sec and the amount suppressed by $1 \mathrm{mM} \mathrm{MnCl}_{2}$ to be 0.022 $\mathrm{p}$-mole $/ \mathrm{cm}^{2}$. sec. This $\mathrm{Ca}$ content corresponding to $0.022 \mathrm{p}$-mole $/ \mathrm{cm}^{2}$. sec was tentatively attributed to the extra entry of $\mathrm{Ca}$ by the generation of potential. But this 


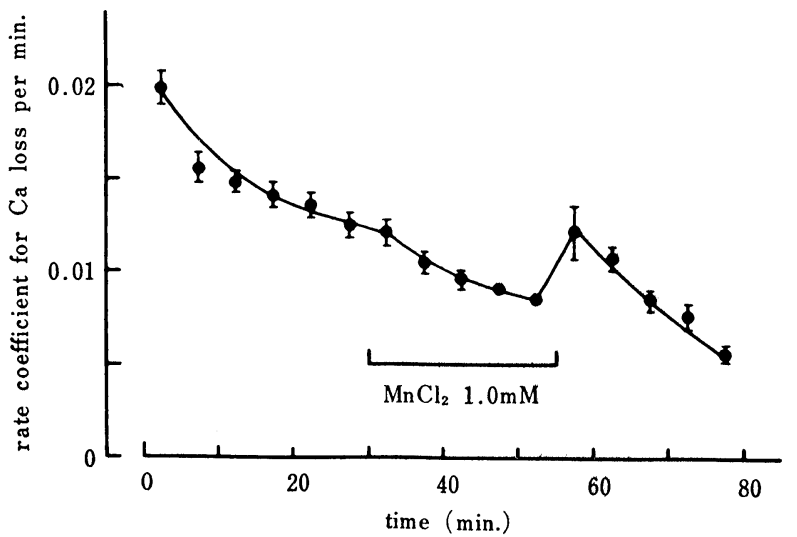

Fig. 2. Effect of $1.0 \mathrm{mM} \mathrm{MnCl}{ }_{2}$ on the rate of loss of ${ }^{45} \mathrm{Ca}$ from taenia strips bathed in the normal Krebs solution. Each point represents mean value + S.E. from 10 preparations. Ordinate, rate of loss of ${ }^{45} \mathrm{Ca}$ : Abscissa, time after beginning of the serial washing.

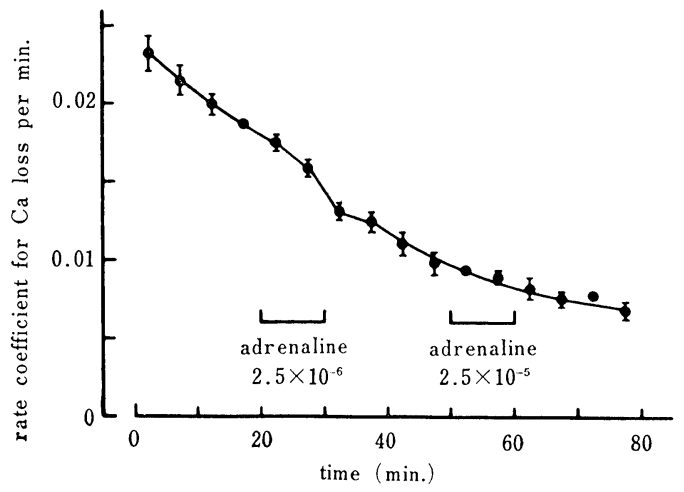

Fig. 3. Effect of adrenaline (first time $2.5 \times 10^{-6} \mathrm{~g} / \mathrm{ml}$, second time $2.5 \times 10^{-5} \mathrm{~g} / \mathrm{ml}$ ) on the rate of efflux of ${ }^{45} \mathrm{Ca}$ from 5 taenia strips.

amount proved to correspond only $2 \%$ of the theoretically calculated necessary amount of divalent cation inflow accompanying the generation of action potential (assuming membrane capacity of muscle cell to be $3 \mu \mathrm{F} / \mathrm{cm},{ }^{2}$ action potential to be $50 \mathrm{mV}$ in height). Thus the present experiment could not give the substantial evidence to the Ca spike hypothesis of the action potential in smooth muscle. 


\title{
The Localization of 5-Hydroxytryptamine and its Participation in Neural Inhibition of Gastrointestinal Smooth Muscle
}

\author{
MICHAEL GERSHON, M.D. \\ Department of Anatomy, Cornell University Medical College, New York, N.Y., \\ U.S.A.
}

It has recently been suggested by Bülbring and Gershon (J. Physiol. 192: $823-846,1967)$ that 5-hydroxytryptamine $(5-\mathrm{HT})$ might participate in ganglionic transmission in the vagal inhibitory pathway to the stomach. This hypothesis was based on the observations that 5-HT activated inhibitory neurons in the myenteric pleuxus, that antagonism of the neural action of 5-HT inhibited vagal relaxation of the stomach and that 5-HT was released from the stomach when the vagus nerves were stimulated, even after asphyxiation of the mucosa.

In order for a substance to be considered a neurotransmitter, however, it is necessary that it be shown to be present in the axon terminals at the synapse in question. At the time Bülbring and Gershon suggested 5-HT might be a transmitter, there was some evidence that 5 -HT was stored in myenteric plexus axon terminals. Following the intravenous administration of the tritiated precursor of 5-HT, 5-hydroxytryptophan (5-HTP), tritiated 5-HT may be found by radioautography in terminal axons in the myenteric plexus (Gershon and Ross, J. Physiol. 186: 477-492, 1966). This observation, however, does not itself confirm the normal synthesis and storage of 5-HT by myenteric plexus neurons.

Since the enzyme, aromatic 1-amino acid decarboxylase, which catalyzes the conversion of 5-HTP to 5-HT, is widely distributed through the mammalian body and occurs outside of the gut, it is necessary to determine whether 5-HT is produced from 5-HTP within the myenteric plexus itself. Since the enzyme is also non-specific, and is involved in the biosynthesis of norepinephrine as well as 5 -HT, it is important to determine whether the accumulation of 5-HT in the myenteric plexus represents an opportunistic accumulation of a "false neurotransmitter" by normally adrenergic axons. Finally, one must determine whether 5 -HT is an endogenous constituent of the myenteric plexus.

The conversion of 5-HTP to 5-HT does occur locally. Segments of mouse stomach and small intesine, incubated in vitro with tritiated 5-HTP, in the presence of pyridoxal phosphate, produce and store 5-HT. The radioactive 5-HT synthesized and stored under these conditions was localized by radioautography. 5-HT was concentrated in mucosal enterochromaffin cells, mast cells, and in a characteris- 
tic location surrounding, but not within, neuronal perikarya of the myenteric plexus.

Beside being able to synthesize 5-HT from its precursor, both stomach and small intestine of mouse and guinea-pig also take up and concentrate 5-HT itself. This concentration is most striking in vitro when segments of gut are incubated with tritiated 5-HT in a concentration of $2 \times 10^{-6} \mathrm{M}$, but can also be demonstrated in vivo.

When intestinal strips are incubated in radioactive 5-HT, a portion of the 5-HT taken up by the tissue appears bound and resists subsequent washout in physiological solution. This tightly bound fraction, which washed out with a rate constant of $0.1 / 100 \mathrm{~min}$, tends to increase with increasing time of incubation.

The structures in the tissue represented by the tightly bound fraction of 5-HT could be located by radioautography. Radioactive 5-HT was found to be taken up and bound by the myenteric plexus of the mouse and guinea-pig, and mast cells of the mouse. No labeling was found in the submucosal plexus, although, histochemically, adrenergic axons are found here. Only mast cells were labeled in the submucosa, and there was no evidence of labeling of periarterial adrenergic axons.

Although the whole small intestine concentrated 5-HT more than the level of the incubating medium, the concentration was about ten times greater in a strip of longitudinal muscle dissected from the intestine with the myenteric plexus adherent to it. Radioactive 5-HT was found by radioautography to be confined to the myenteric plexus in these strips.

In order to determine whether 5-HT is naturally present in the myenteric plexus, 5-HT was extracted from the innervated longitudinal muscle strips and measured by the ninhydrin method of Snyder, Axelrod and Zweig. 5-HT was found to be present in the strips but the concentration was very low, (30 ng/g). The presence of 5-HT was confirmed by thin layer chromatography. 5-HT was identified by its migration and by its characteristic yellow fluorescence after condensation with formaldehyde gas.

The presence of monoamines in the myenteric plexus was also studied by a modification of the fluorescence histochemical method of Falck and Hillarp. The longitudinal muscle and adherent myenteric plexus were stripped from the guinea pig small intestine, dried over phosphorus pentoxide on slides as whole mounts, and subjected to formaldehyde vapor generated from paraformaldehyde at $80^{\circ} \mathrm{C}$.

In control preparations, most of the fluorescence was due to the presence of norepinephrine. Norepinephrine was depleted by reserpine or alpha methyl p-tyrosine. This depletion was associated with a loss of the green fluorescence associated with catecholamines. When these drugs were followed by inhibition of monoamine oxidase by pheniprazine or tranylcypromine, a widespread yellow fluorescence was seen in the myenteric plexus. Since the appearance of the yellow fluorescence was prevented by inhibition of 5-HT synthesis with parachlorophenyl- 
alanine, it is probable that the fluorescence was due to 5-HT.

It thus appears that 5-HT can be synthesized locally from 5-HTP by the myenteric plexus. It can also be taken up and stored against an adverse concentration gradient. In both these respects, the terminal axons of the myenteric plexus appear to differ from those of the adrenergic perivascular and submucosal plexuses. Finally, 5-HT seems to be a normal constituent of the myenteric plexus, but it is present in very small amounts.

The technical assistance of Mrs. Judith Luckower is greatefully acknowledged. This research is supported by grants NB 07436 and NB 05539 


\section{Significance of Pyloromyotomy and Pyloroplasty}

TETSUO MAKI, M.D.

Department of Surgery, Tohoku University School of Medicine, Sendai, Japan

It has been known that the occurrence of delayed gastric empyting due to abnormal excitement of the pyloric portion is very common following segmental and proximal gastric resections which retain the pyloric ring. To facilitate the better gastric emptying, it becomes necessary to add a drainage procedure such as pyloromyotomy or pyloroplasty. In performing pyloromyotomy or pyloroplasty, one may yet raise questions concerning the proper length of an incision, functional difference between these procedures, or relating to the indication or selection of these drainage procedures. To meet these questions, we have done a series of experiments and obtained the following results.

\section{Materials and Methods}

Adult mongrel dogs weighing about $15 \mathrm{~kg}$ were used for the experiments. Experiment 1. As the control, the stomach was exposed through an upper midline abdominal incision under thiopental sodium anesthesia after 24 hours fasting. Two bipolar silver-electrodes were used for the recording of electromygoram. One electrode was fixed on the anterior wall of the stomach $1 \mathrm{~cm}$ proximal to the pyloric ring and close to the greater curvature. Another electrode was fixed $4 \mathrm{~cm}$ proximal to the pyloric ring. To measure the contractile force of the pyloric ring area, a rubber balloon measuring $1 \times 1 \times 3 \mathrm{~cm}$ was introduced and placed inside of the pyloric ring through small two incision wounds made on the antrum and the duodenum. A polyethylene tube from the balloon was connected to a strain-gauge transducer. For the recording of electromyogram and contraction pressure, we used a multi-channel electromyograph made by San-ei Instrument Co. with penwriting recorder.

As the pyloromyotomy, a Ramstedt type incision with $3 \mathrm{~cm}$ in length was performed in the following two groups of dogs. In the first group, we studied the effect of pyloromyotomy done on the normal stomach which had not been subjected to any preceeding preparations. In the second group of dogs, we studied the effect of pyloromytomy on the abnormally excited pyloric portion produced by transection of stomach between corpus and antrum after the Sugawara procedure. ${ }^{1)}$

Experiment 2. In the next place, the stepwise pyloromyotomy incisions were 
made starting from the pyloric ring. In the first step, incision was made for $1 \mathrm{~cm}$ and then the line of incision was extended proximally $1 \mathrm{~cm}$ each in the following two steps. In each step, we recorded the contraction pressure and electromyogram of the pyloric portion. In other experiment, we reversed th direction of incisions starting at the point $3 \mathrm{~cm}$ proximal to the pyloric ring and extended the incision in three steps until it reached to the pyloric ring.

Experiment 3. In the present experiment, we attempted to evaluate the effect of pyloroplasty of the Heineke-Mikulicz type. A longitudinal incision, $4 \mathrm{~cm}$ in length, was made $3 \mathrm{~cm}$ on the antrum and $1 \mathrm{~cm}$ on the duodenal side.

\section{RESULTS}

Experiment 1.: Our recent understanding is that, electromyographically, discharge interval is prolonged and the propagation velocity increases as the gastric motility becomes more active, and that the incidence of anti-peristaltic discharges becomes high when the pyloric portion is abnormally excited. Clinically, it is not uncommon to see spasm-like contraction of the pyloric portion accompanied with anti-peristalsis.

Therefore, we regarded the frequent occurrence of anti-peristaltic discharges as an index or abnormal excitement of the pyloric portion, and also took the prolongation of the discharge interval and increased propagation velocity as indices of hypermotility of the stomach.

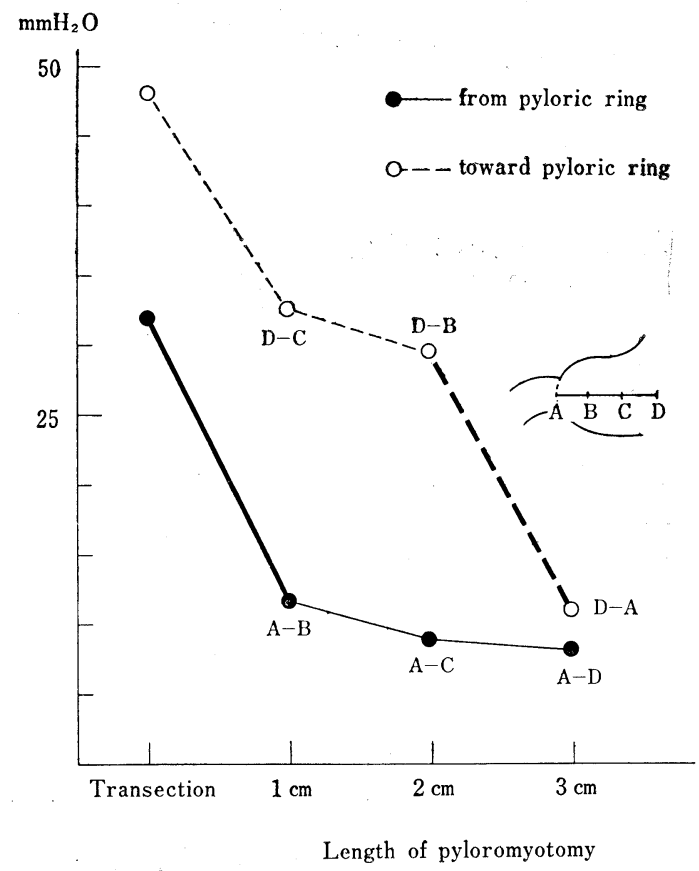

FrG. 1. Stepwise pyloromyotomy and contraction pressure 
Electromyographically, an addition of pyloromyotomy on the normal canine stomach showed the tendency of decreased pyloric motor function. In other words, it showed the weakening of propelling force of the stomach content. Immediately after transection, anti-peristaltic discharges were developed in the pyloric portion at the rate of 80 per cent of all discharges recorded. However, an addition of pyloromyotomy with $3 \mathrm{~cm}$ in length markedly reduced the incidence of antiperistaltic discharges as low as 30 per cent.

Experiment 2.: Figure 1 shows the detailed analysis of the findings of the experiment 2. Transection prior to additional pyloromyotomy produced a sharp rise in contraction pressure up to $32 \mathrm{~mm}$ of water. When the pyloromyotomy incision, $1 \mathrm{~cm}$ in length, was made on and from the pyloric ring, contraction pressure fell to $11.5 \mathrm{~mm}$ of water. Extension of the incision line, $1 \mathrm{~cm}$ each in the following two steps resulted in further reduction of the pressure to 9 and $8.5 \mathrm{~mm}$ of water respectively. Conversely, stepwise extension of the pyloromyotomy incision toward the pyloric ring gradually reduced the contraction pressure from the control value of 48 to 32.5 and further down to $29.5 \mathrm{~mm}$ of water after two steps of $1 \mathrm{~cm}$ incision. Lastly, $1 \mathrm{~cm}$ extension over the pyloric ring resulted in the sudden fall of pressure to 11 $\mathrm{mm}$ of water.

Changes in the incidence of anti-peristaltic discharges are seen in Fig. 2. Before

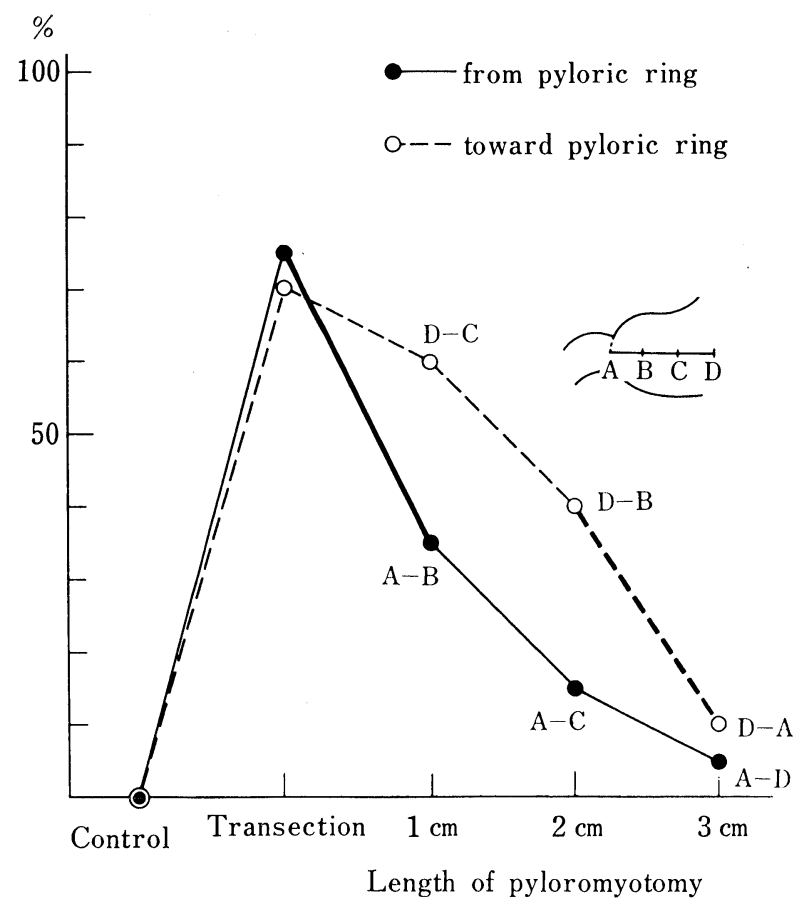

Fra. 2. Stepwise pyloromyotomy and incidence of anti-peristaltic discharges 
transection, pyloromyotomy gave no significant influence and there was seen no incidence of anti-peristaltic discharge. After transection, anti-peristaltic discharges were seen at the rate of $75 \%$. One centimeter pyloromyotomy over the pyloric ring reduced the incidence of anti-peristaltic discharges as low as $35 \%$ and extension of an incision to proximal 2 and $3 \mathrm{~cm}$ in total length further but gradually reduced the rate down to $15 \%$ and $5 \%$ respectively.

On the other hand, when the stepwise extension of an myotomy incision was done toward the pyloric ring, corresponding rates of anti-peristaltic discharges were; $70 \%$ after transection, $60 \%$ after $1 \mathrm{~cm}$ pyloromyotomy, $40 \%$ after total of $2 \mathrm{~cm}$ incision and $10 \%$ after total of $3 \mathrm{~cm}$ incision. These data clearly show that it is most effective for pyloromyotomy when incision is made right over the pyloric ring. It is also known that the extension of an incision apart from the pyloric ring further but gradually reduces the pyloric contraction pressure. Findings of electromyography also showed quite similar tendency.

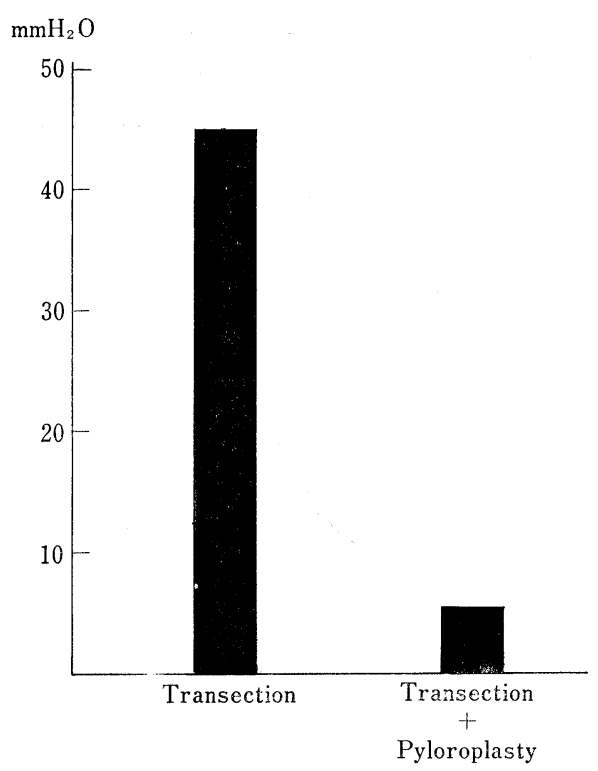

FIG. 3. Pyloroplasty after gastric transection (contraction pressure)

Experiment 3.: As shown in Fig. 3, decrease in contraction pressure of the pyloric ring area in the cases of pyloroplasty was noticed more markedly than that of pyloromyotomy cases. Pressure decreased from $45 \mathrm{~mm}$ of water at the time of transection to $5.5 \mathrm{~mm}$ of water after an addition of pyloroplasty. Therefore, it is evident that the abnormal excitement and high contraction pressure of the pyloric ring area is weakened by the addition of pyloroplasty. 


\section{Comment}

The experimental results of pyloromyotomy lead to the following three conclusions. First, pyloromyotomy suppresses the state of abnormal excitement of the pyloric portion and decreases the contraction pressure of the pylorus. Second, if there exists an independent sphincter muscle in the pyloric ring, there will be no difference between the myotomy of the pyloric ring muscle alone and the myotomy including the muscles of pyloric canal and antrum. The results obtained in the present experiments suggest that the pyloric ring does not possess an independent sphincteric function, but it is merely the terminal portion of the stomach with the strongest sphincteric function. Lastly, pyloromyotomy brings about little drainage effect when the length of an incision is not long enough or when it failed to divide the circular muscle of the pyloric ring. In the clincial experiences, it was also revealed that the occurrence of gastric stasis is inevitable when the length of an incision was less than $2 \mathrm{~cm}$, while an incision of 3 to $5 \mathrm{~cm}$ long resulted in a sufficient drainage effect. Thus, the fundamental signfiicance of pyloromyotomy seems to be in it's reducing effect of excitement and contractile force of the pylorus.

Next, the most remarkable effect of pyloroplasty is seen when it is performed on the stomach which shows abnormal excitement of the pyloric portion with high contraction pressure. The significance of pyloroplasty is in it's effect in sacrificing the sphincteric function of the pylorus and widen its lumen which results in greater drainage effect.

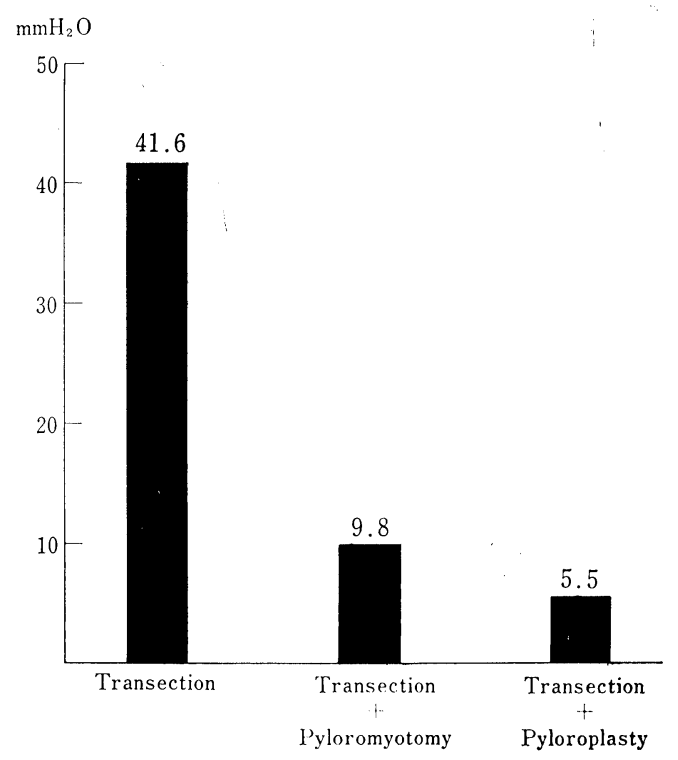

FIG. 4. Effect of drainage procedures on contraction pressure 
Lastly, I would like to mention about indication of pyloromyotomy and pyloroplasty. As shown in Fig. 4, reducing effect of the high contraction pressure seen at the time of gastric transection is greater by pyloroplasty than by pyloromyotomy when the pyloric region was incised in the same length. Same tendency is confirmable from the study of electromygoram concerning the reducing effect of pyloric excitement. Namely, it is well comprehensible that pyloroplasty has a bigger drainage effect than pyloromyotomy. For this reason, pyloroplasty is best indicated when severe excitement of the remaining pyloric portion is present and the most powerful drainage effect is desired even by sacrificing the sphincteric function of the pylorus. On the other hand, pyloromyotomy has an indication when the less drainage effect seems sufficient and preservation of the sphincteric function of the pylorus is needed.

\section{Reference}

1. Sugawara, K.: An electromyographic study on the motility of canine stomach after transection and end-to-end anastomosis. Tohoku J. exp. Med., 84, 113-124, 1964. 
Jap. J. Smooth Muscle Res., 6, 123 128, 1970

\title{
The Discharge of Bile into the Duodenum and Electrical Activities of the Muscle of Oddi and Duodenum.
}

\author{
KEIICHI ONO, M.D. \\ Department of Surgery (Prof. K. Oh-Uti), Faculty of Medicine, Hirosaki \\ University, Hirosaki, Japan
}

Although the anatomical study of Boyden ${ }^{1)}$ has demonstrated a distinct sphincter at the terminal portion of the common bile duct, a few investigators still doubt whether the sphincter of Oddi functions independently of the duodenal musculature or not. Therefore, the mechanism of flow of bile into the duodenum is still a matter of concern to practicing physicians as well as to basic investigators in anatomy, physiology, and pharmacology.

Ishioka ${ }^{2)}$ in 1959 demonstrated the difference in electrical activities between the muscle of Oddi and the duodenal wall muscle in rabbits suggesting the former to be functionally indpendent of the latter.

The present study was made to investigate the details of correlation between the discharge of bile into the duodenum and electrical activities of the muscle of Oddi and the duodenum.

\section{Materials and Method}

\section{Animal Experiment}

Twenty-five rabbits were studied as subjects.

Laparotomy was done by right subcostal incision under local anesthesia. A bipolar electrode of Ishioka ${ }^{2}$ sewn in the muscle of Oddi and another electrode was implanted into the anterior wall of the duodenum at the level of the sphincter of Oddi.

A No. 3 Nelaton's cathether was inserted into the common bile duct by supraduodenal choledochotomy and was connected to an electronic manometer for measurement of intracholedochal pressure. The common bile duct was irrigated with saline solution or a contrast medium through the catheter. A rubber balloon of $2.0 \mathrm{~cm}$ in diameter was placed in the duodenum through a suprapyloric gastrotomy and was filled by saline solution. This balloon was connected to the electronic manometer for measurement of intraduodenal pressure.

Action potentials from these muscles and these pressures were conducted and recorded simultaneously by a DC amplifier with ink writers. 
At the same time biliary cinecholangiography was performed by an image intensifier with the $16 \mathrm{~mm}$ cinecamera.

\section{Clincial Ohservation}

The subjects studied were 22 patients with gallstone diseases operated upon in our department. Immediately after cholecystectomy, choledocholithotomy, or both, a Béniqué's metal sound was inserted into the common bile duct toward the duodenum to identify the choledochoduodenal junction, the muscle of Oddi. The outside serosa of the descending duodenum was then stripped from the retroperitoneum and turned over to the left. Holding firm the helve of the metal sound with the left hand, the terminal choledochus including the muscle of Oddi was precisely located bimanually by feeling the tip of the sound with the right hand through the doudenal wall. A bipolar needle electrode fumigated with formalin before operation was sewn in the muscle of Oddi as shown in Fig. 1. After reverting the duodenum to the original state, another bipolar electrode was then implanted into the anterior wall of the duodenum at the level of the sphincter of Oddi. Following these procedures, a T-tube was set in the common bile duct. The incis-

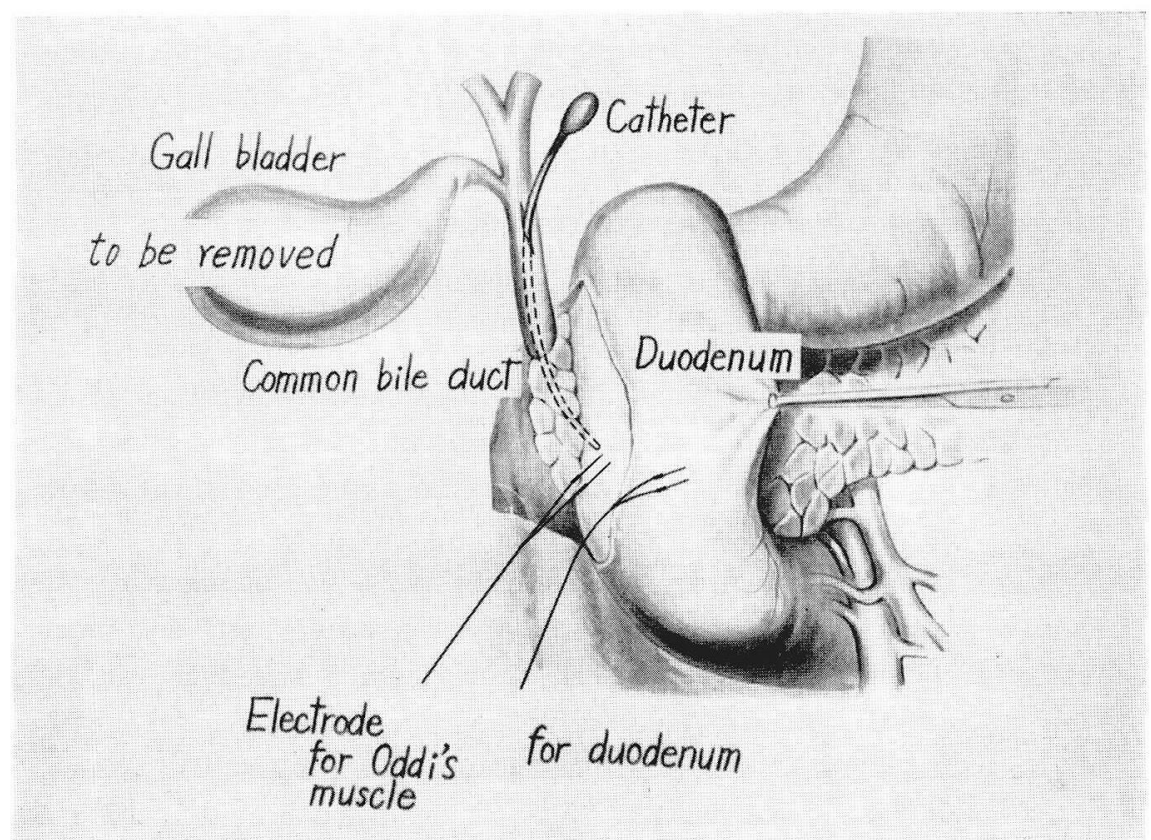

FIG. 1. A metal sound inserted into the common bile duct toward the chledochoduodenal junction and a bipolar needle electrode is then, by duodenal mobilization bimanually, fixed by suture in the muscle of Oddi at the terminal choledochus with their tips inserted in the muscle. Another electrode is simultaneously implanted into the anterior duodenum at the level of the muscle of Oddi. 


\section{CINE-OSCILLO-SYNCHRONIZING-UNIT}

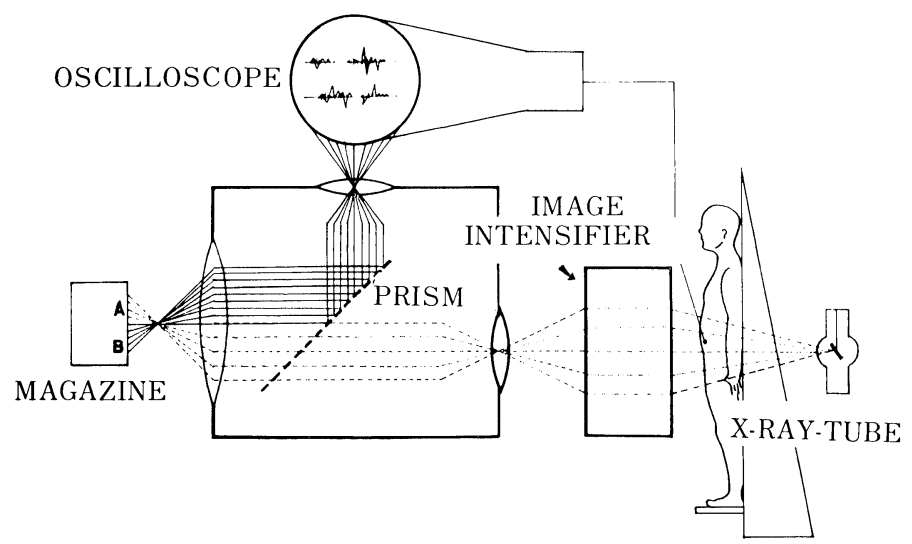

Fra. 2 The simultaneous recording system

ion was closed and the lead wiring of electrodes drawn from the sutured wound was then covered aseptically with sterile gauze. The electrodes implanted were left inlying over the ensuing period of two to three weeks to record the electrical potentials from time to time. The electrodes were then withdrawn: there were no complications associated with the inlying electrodes or following their removed in any of the patients studied.

Action potentials from these muscles were conducted by a two-channel DC amplifier with ink writers for 5 to 17 days after the operation. At the same time a fluorographic observation of the biliary system was performed by infusing $\mathbf{a}_{\mathbf{c}}$ contrast medium into the common bile duct through the T-tube. The electrical activities of these muscles displayed on the oscilloscope and biliary radiogram taken by an image intensifier were simultaneously recorded on a $16 \mathrm{~mm}$ cinefilm with a cine-oscillo-synchronizing unit as shown in Fig. 2. The two sets of data recorded on each frame of the film were enlarged on the screen of a film motion analyzer and measured accurately by a scale.

\section{Results}

In rabbits discharge pattern of the action potential from the muscle of Oddi was obviously different from that of the duodenal wall muscle. Both the spike discharges were closely related to raising the intracholedochal and the duodenal pressure respectively as shown in Fig. 3 .

Biliary radiocinematogram revealed that the contrast medium into the duodenum was interrupted abruptly corresponding with the appearance of the spike discharge from the muscle of Oddi and was increased during the resting state of the spike discharge. On the other hand, the contrast medium kept 

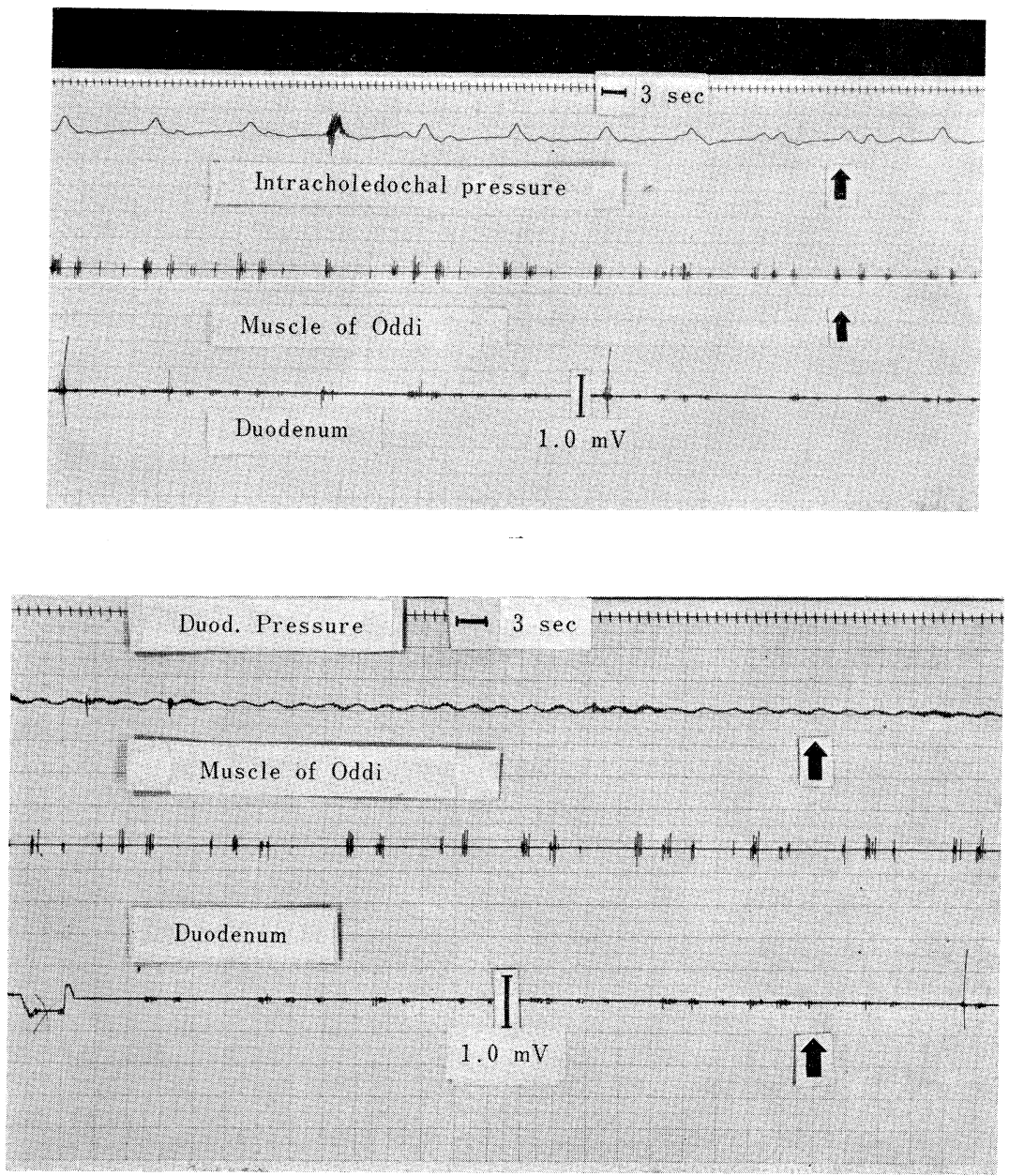

B

Frg. 3.

(A) Correlation between the intracholedochal pressure and the spike discharge from the muscle of Oddi

(B) Correlation between the duodenal pressure and the spike discharge from the duodenal wall muscle

flowing into the duodenum without interruption even when the spike discharge from the duodenal wall was recorded.

In the clinical observation electrical activities of the muscle of Oddi and the duodenum were recorded almost simultaneously, but they were different in the detailed discharge pattern. When an electrical stimulation was given to the muscle of Oddi, the action potential was markedly accelerated and the intraductal pressure was elevated, but no change of the discharge pattern was seen in the duodenum. Reversely, when the same stimulation was given to the duodenum, 
the electrical activity was markedly accelerated in the duodenum, but the muscle of Oddi was not influenced and also the intraductal pressure was not elevated. Bile flow was interrupted at the terminal portion of the common bile duct when electrical activity of the muscle of Oddi was observed. When bile was poured into the duodenum, electrical activity of the muscle of Oddi was not observed. Bile flow was not interrupted by the electrical activity of the duodenal wall muscle.

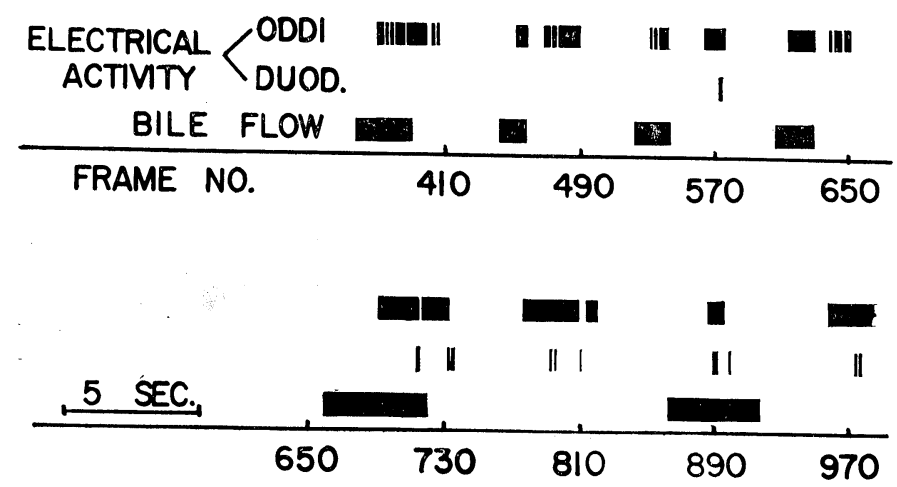

FIG. 4. Correlation between bile flow and electrical activity of the Oddi's muscle and duodenum.

An accurate measurement of a consecutive series of the recorded film using the film motion analyzer revealed that bile flow is stopped by the preceding electrical activity of the muscle of Oddi and is not essentially influenced by the electrical activity of the duodenal wall as shown in Fig. 4.

\section{Discussion}

From the results obtained in animal experiment mentioned above it is conceivable that the spike discharge from the muscle of Oddi originates in a contractive action of the same portion and this contraction composes the terminal choledochal pressure and prevents the regurgitation of the duodenal content into the common bile duct.

It would follow from the clinical observation mentioned above that spike discharge of the muscle of Oddi eventuates in any arrest of the flow of bile at the terminal choledochus into the duodenum. In addition, the spike discharge occurring in the duodenal wall at the level of the muscle of Oddi was found not always to cause cessation of the flow.

\section{SƯmMARY}

From the above observations it is concluded that the muscle of Oddi has a sphincteric mechanism and acts independently of the activity of the duodenal wall 
muscle. These findings are considered to be consistent with the view of Boyden who concluded that relaxation of the muscle of Oddi permits the discharge of bile into the intestine.

\section{References}

1. Boyden, E.A. The anatomy of the choledochoduodenal junction in man. Surg. Gynec. Obstet., 104, 641-562, 1957.

2. Ishioka, T. Electromyographic study of the choledochoduodenal junction and duodenal wall muscle. Tohoku J. exp. Med., 70, 73-84, 1959. 


\title{
Human Electrogastrography
}

\author{
SHOICHI KOHATSU, M.D. \\ Department of Surgery, Stanford University School of Medicine, \\ Veterans Administration Hospital, Stanford, California, U.S.A.
}

Early studies of human electrogastrography did not stress rate analysis, but more recent studies of the gastric slow waves in both $\operatorname{man}^{2,4}$ and animals ${ }^{1,3,5}$ have demonstrated the significance of rate analysis. Human electrogastrograms have been recorded using cutaneous, swallowed, or implanted electrodes, but for general clincial use, the cutaneous electrode would be most desirable if they produce adequate, accurate recordings for rate analysis. This study was undertaken to 1) evaulate the silver-silver chloride cutaneous electrodes, 2) establish the fasting gastric electrical rate of normal subjects, and 3) determine the gastric rate changes with eating.

Electrograstrograms were obtained from 30 subjects who had no known gastroduodenal disease. There were 12 females and 18 males, and their ages ranged from 23 to 68 years. Nine of these subjects had carcinoma of the thoracic esophagus, and they underwent exploratory celiotomy prior to treatment with external irradiation. At operation, 2 to 3 stainless steel electrodes were implanted into the gastric antral muscle parallel to the circular layer, and sutured securely. These electrodes were made of Teflon coated, stainless steel wire with an exposed, sharpened tip of approximately $3 \mathrm{~mm}$. Each electrode was brought out separately through the abdominal wall. The patients were studied following recovery from the celiotomy, and were fasted for at least 12 hours prior to each test. Monopolar recordings were obtained with the reference electrode attached to the thigh. The signals were passed through a filter with a pass band of $0.03-15 \mathrm{~Hz}$, amplified, and recorded on a multichannel penwriter, and also magnetic tape. Recordings were also obtained simultaneously from cutaneous silver-silver chloride electrodes attached on the abdomen. A fasting trace was recorded for 30 to 60 minutes, and the subject then ate a light breakfast. Recordings were continued during eating and for 1 to 2 hours following completion of the meal. The implanted electrodes did not produce any discomfort to the patients, and were removed after 2 to 3 weeks without any complication.

An additional 21 normal subjects were tested using an array of 4 to 6 cutaneous silver-silver chloride electrodes applied to the upper quadrants of the abdomen, and recorded bipolar using the combinations of electrodes yielding the best recordings. 
The electrogastrograms were analyzed for rate differences and changes.If the recordings were free enough from noise and artifacts, they were analyzed for mean rates by direct hard measurement. Mean rates were retrieble from noisy recordings by autocorrelation using the signals recorded on magnetic tape, and a digital computer. The mean fasting rate and the rates of alternate five minute periods following eating were analyzed. Student's " $t$ "-test was used for statistical analysis, and the changes were considered significant if $p$ was $<.05$.

The implanted electrodes produced clean recordings, and over $90 \%$ of the subjects studied with cutaneous electrodes yielded usuable recordings. Comparison of the recordings obtained simultaneously from the implanted and cutaneous electrodes revealed that the latter electrodes produce accurate, adequate traces for rate analysis, as there was a consistent 1:1 time relationship of the waves recorded from these electrodes (Fig. 1).
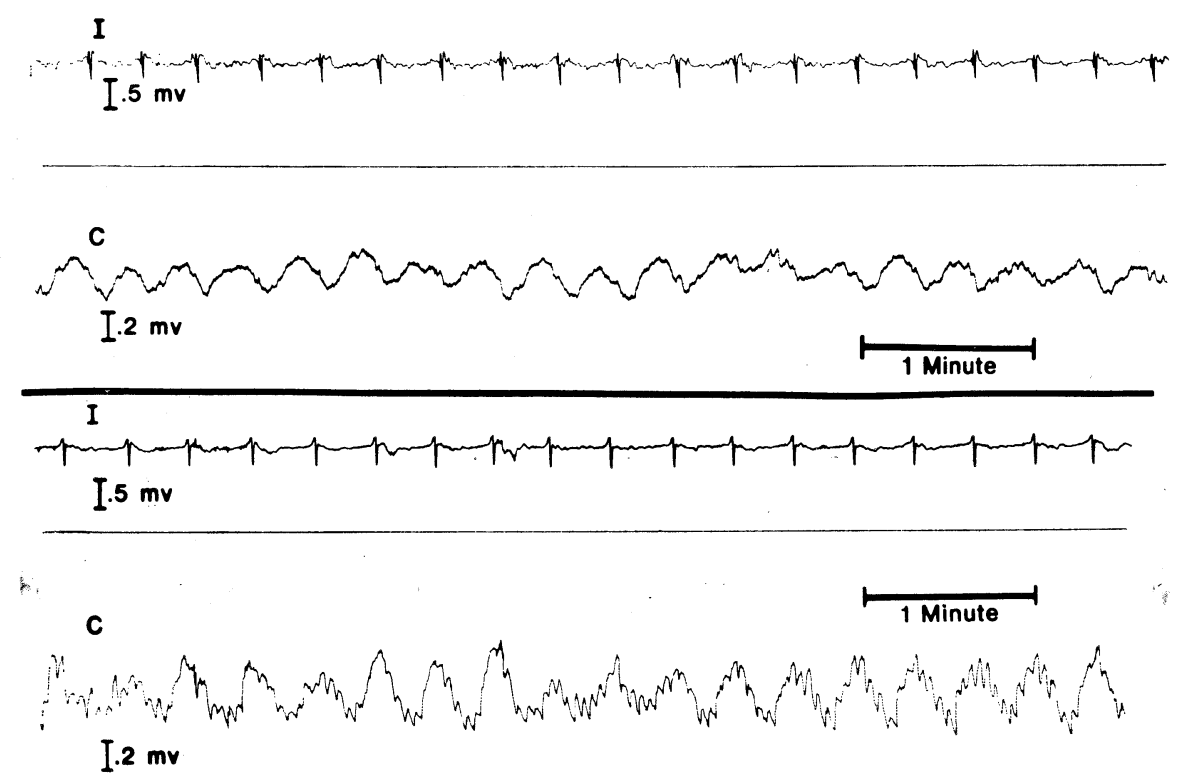

Frg. 1 Simultaneous electrical recordings from implanted stainless steel electrodes (I) and cutaneous silver-silver chloride electrodes (C) from 2 patients. Note the 1:1 time relationships of the waves recorded from the cutaneous and implanted electrodes.

The mean fasting rate was $2.9 \pm .3$ cycles per minute. Eating produced a definite pattern (Fig. 2). Following eating, there was a significant decrease in rate to 2.5 cycles per minute (average $8 \%$ decrease) which occurred almost immediately. Every test showed this significant decrease in rate by the end of five minutes following completion of the meal. Some of the recordings during eating were suitable for analysis, and they showed a decrease in rate within a minute or two after initiation of eating. Following this initial decrease, there was an increase in 


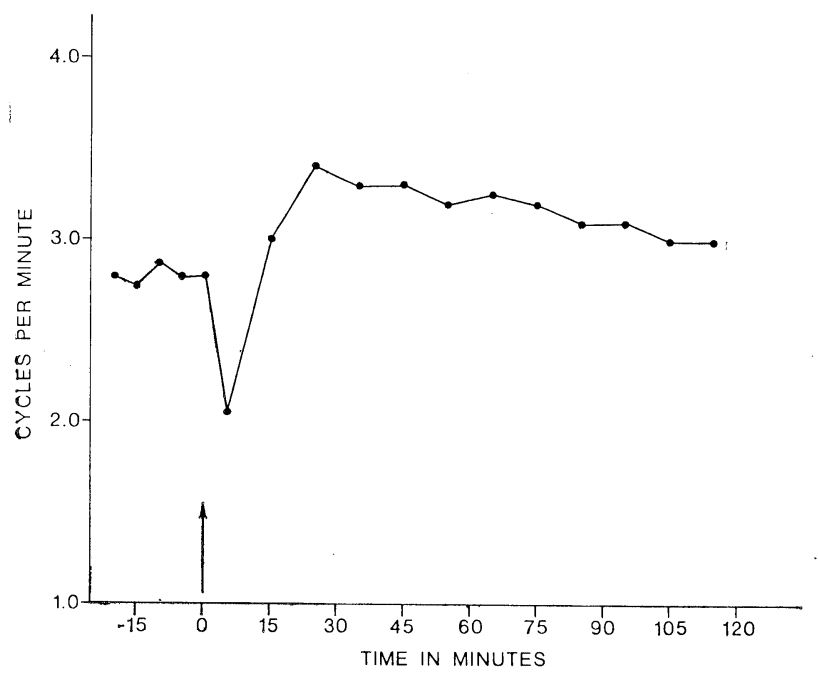

Frg. 2 Gastric rate response to eating in man. Each point represents the frequency of a 5 minute period.

rate above fasting reaching the maximum frequency within 30 minutes (average 28 minutes). There was then a gradual decrease in rate approaching the fasting rate over the one to two hours of recording. All of the electrogastrograms showed this pattern of response to eating.

Studies of the gastric electrical waves in both man and animals suggest that by applying rate analysis to human electrogastrography, this technique could be used to study gastric motor physiology and pathophysiology of various gastrointestinal disorders. In dogs, vagal stimulation with insulin hypogylcemia produces an increase in the frequency of the slow waves, and vagotomy abolishes this response. ${ }^{1}$ Vagotomy also produces a decrease of the fasting rate and an increase in rate variation. Feeding results in a definite pattern of electrical rate changes ${ }^{1)}$ which is similar in general configuration but different in time course to that in man. Transection of the mid or distal stomach with re-anastomosis produces a lowering of the slow wave frequency distal to the transection without changing the proximal rate in dogs. ${ }^{5}$ In the distal segment, reverse propagation of the slow wave is also common. A Heineke-Mikulicz pyloroplasty tends to restore the propagation to the normal direction. ${ }^{3} \mathrm{Oi}$, Tanaka, and $\mathrm{Iwai}^{2}$ ) reported that in man, the frequency of the electrical waves parallel the gastric acidity, and the frequency is high in patients with duodenal ulcer and low in patients with gastric carcinoma.

Electrogastrograms adequate for rate analysis can be obtained with swallowed, implanted, or cutaneous electrodes. The implanted electrodes yield the best recordings, but the obvious disadvantage is that an operation is necessary. The swallowed intragastric electrodes with suction produce excellent recordings, but the swallowing of the tube may cause nausea or the tube within the stomach may 
act as a stimulus. In addition, the suction necessary to produce the stable electrode-mucosal junction can be applied only for a limited time. The cutaneous electrodes are simple to use and there is no discomfort for the subject. Long-term studies are possible with these electrodes. The quality of the recordings is not as good as those obtained from implanted or swallowed electrodes, but this study demonstrates that the silver-silver chloride cutaneous electrodes produce adequate, accurate electrogastrograms for rate analyses. In addition, eating produces a characteristic response of the gastric rate, and rate analysis is important in human electrogastrography.

\section{References}

1. Nelsen, T.S., Eigenbrodt, E.H., Keoshian, L.A., Bunker, C. and Johnson, L. Alterations in muscular and electrical activity of the stomach following vagotomy. Arch. Surg., 94: 821-835, 1967.

2. Oi, M., Tanaka Y. and Iwai, Y. Electrogastromyography. Digest 7th Int. Conf. Med. Biol. Eng. (Stockholm), 335, 1967.

3. Shiratori,T ., Sugawara, K., and Kuroda, S. Surgical signficance of pyloroplasty with special reference to electromyographic findings. Tohoku J. Exp. Med., 85, 192200, 1965.

4. Shiratori, T., Okabayashi, T., Harata, N. and Nagaoka, K. Intraluminal lead of the gastric action potentials in human subjects: use of a new suction needle electrode. Surgery, 66: 483-487, 1969.

5. Sugawara, K. An electromygoraphic study on the motility of canine stomach after transection and end-to-end anastomosis. Tohoku J. Exp. Med., 84: 113-124, 1964. 


\title{
Clinical Electrogastrography
}

\author{
YOSHIKI TANAKA, M.D. AND MINORU OI, M.D. \\ Department of Surgery, Jikei University School of Medicine, T'okyo, Japan.
}

This paper will be described only about the electrogastromyography of human cases. However, it should be mentioned at first that the electromyogram of canine stomach has the almost same characteristics as human ones.

For exmaple, the electromyogram of the canine stomach has potential change of spike like shape as it is known well. It is same as $\mathrm{R}$ wave named by Bozler, ${ }^{1)}$ same as pace setter potential of Code $^{2}$ ) and same as initial potential of Daniel. ${ }^{3}$ ) While it has been called "spike" or "slow spike" by authors, because it has such a long duration as 1 second or more. Moreover, it has a characteristics as like as intestinal slow wave. About this point it will be mentioned later.

The frequency of the canine gastric (slow) spikes is about 4 per minutes. The spike occurs at the border line between upper one third and lower two thirds of the gastric body. And it propagates downward toward the pylorus increasing its amplitude in advance of visible peristalsis. But, the amplitude drops suddenly at $5 \mathrm{~mm}$ zone adjacent to the pylorus. And the pylorus has a "silent zone" without any action potentials, as Bass and Code ${ }^{4)}$ have called the zone "insulatory zone".

All these characteristics are observed on human stomachs. And they are almost same, although some very little differences are recognized between human and canine cases. Then let us change the subject to the human electrogastromyography.

\section{Extra Canal Electrogastromyography}

Before applying the intra canal electrogastromyography on the patient, the authors tried at first the extra canal electrogastromyography during operation under the thiopenthal- $\mathrm{N}_{2} \mathrm{O}$ anesthesia without any premedications. As our preliminary studies showed, the thiopenthal- $\mathrm{N}_{2} \mathrm{O}$ anesthesia has no effect upon the electrogastromyogram.

The electrode was a needle bipolar one consisting of two silver needles of which the diameter was $170 \mu$ each, and spacing was $1.5 \mathrm{~mm}$. This electrode was same as used on canine experiment. And it was inserted muscular layers through gastric serosa.

Thirty-one cases with various gastric deseases were examined. The rhythmical discharges forming simple slow spikes as Fig. 1 were recorded. Its duration is longer than canine's. It is almost two seconds. Because of its shape 


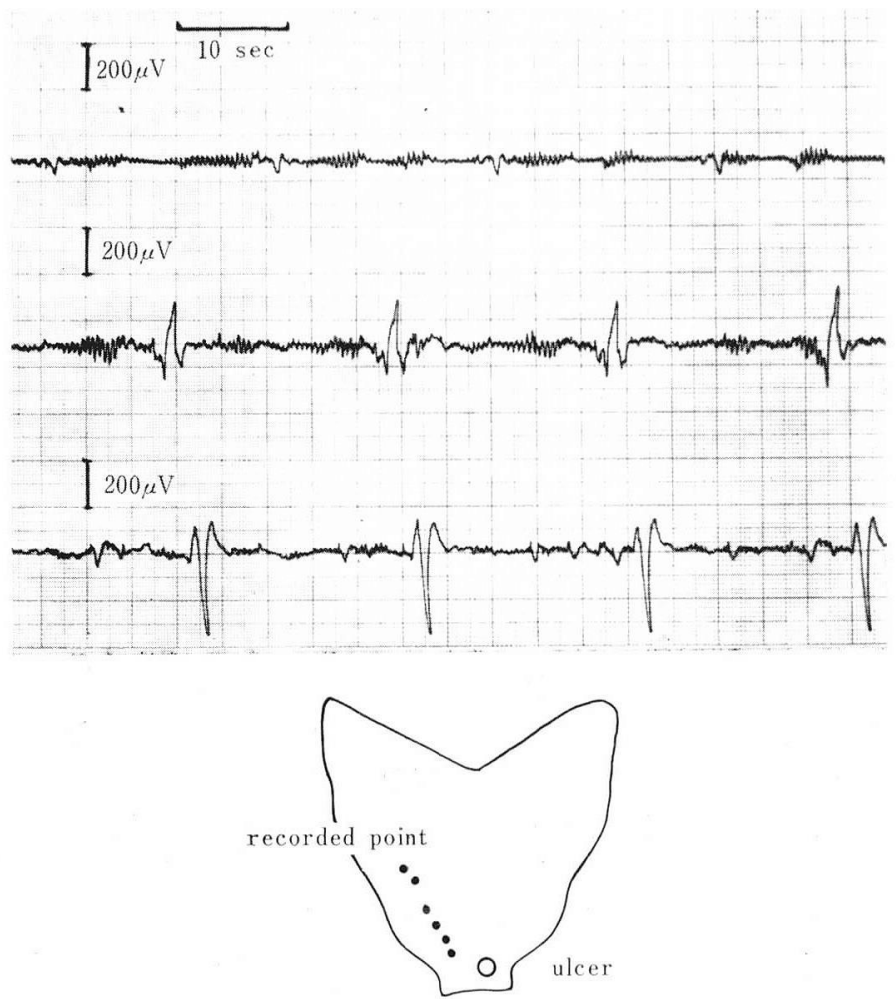

E. S., 40yrs, male duodenal ulcer

FIG. 1. A typical pattern of extra canal electrogastromyogram (9 years ago the authors called it gastro-electromyogram)

and characteristics the authors have called it "slow spike" or "spike" for short, although it is not real spike of physiologists. For the reasons, the slow spike was recorded whenever the peristalsis was visible or not. Differing from canine stomachs on human stomachs almost cases had no visible peristalsis under the laparotomy. However, when the peristalsis was visible the spike occured in advance of the peristalsis as like as canine ones. This finding is seemed to be same as the slow wave of the intestine. And the authors have never surely discriminated the real fast spike or spike burst on the electrogastromyogram of men as well as canines in ordinary conditions. This is the reason why the authors call the potential changes of the stomach "slow spike", as it seems to combine the characteristics of real fast spike and slow wave of intestine.

It is very important that the gastric slow spike can be recorded always, namely whenever the peristalsis exists or not. And this was the chief reason the authors ${ }^{5}$ believed if the slow spikes were marked the intra canal electrogastromyography would be certainly successful without laparotomy. Moreover, the spikes occur 
constantly, and it is not changed by ordinary factors, for instance, the administration of ordinary dose of atropine has no effect on the spikes. This fact is considered also to be advantageous for intra canal electrogastromyography.

On the extra canal electrogastromyography, the human gastric slow spike occured at the midline of the gastric body, that was lower than occuring ine of the canines, and the spike propagated downward towards the pylorus as like as canine's. The velocity of the propagation was $5.7 \mathrm{~mm}$ per seconds on an average. And usually the velocity in the antrum was faster than that in the body. And there was no remarkable action potential at the pylorus. At the pylorus man has also "silent zone" which is considered to consist of the connective tissue zone between antral and duodenal circular muscles.

The amplitude of the spikes was higher at the antrum ( $352 \mu \mathrm{V}$ on an average) than at the body $(146 \mu \mathrm{V}$ on an average). Of course the amplitude on bipolar method is not acknowledged so reliable, because of antagonistic action by push pull effect of bipolar leading. However, there were some concentration of amplitude among the 132 cases of canine stomachs in according with each portions of the stomachs. And the amplitude had a tendency that the portion was lower the amplitude was higher except only the silent pylorus. At this point the human stomach had quite same tendency as canine's. Although, the value of spike amplitude of human stomachs did not so concentrate as canine's. For exmaple, at the antrum the amplitude distributed from 80 to $700 \mu \mathrm{V}$ in human cases. On the other hand in canine cases it was only from 900 to $2300 \mu \mathrm{V}$. Of course it will call fresh studies for the future, the authors suppose that as there are different kind of diseases in the stomachs of the human cases, so the wide distribution is obtained. However, at present it cannot be tried to analyze any more.

Then, the frequency of the spikes must be analyzed. The frequency is constant without regard to various portions of the stomach so far as the spike can be recorded. Because the spike occurs regularly at the midline of the body and propagates towards the pylorus without interruptions. And the bipolar leading does not have an influence on the frequency. Therefore, the frequency has been chosen as a principal index especially for coming intra canal electrogastromyography.

The frequency of 31 examined cases distributed from 2.3 to 6.6 per minutes. And their average value was 3.2 per minutes. The cases of duodenal ulcer showed the highest value of the frequency among different gastric diseases. The average value of duodenal ulcer cases was 3.5 per minutes. And the lowest was 2.8 of gastric ucler cases.

The 21 patients of total 31 cases were examined the gastric juice. And the frequency of the spikes showed a tendency to be in parallel with the value of gastric acidity. In the cases of hyperacidity the value of the frequency was 3.3 per minutes and the hypoacidity cases showed 2.9 (Table 1). This result points out 
TABLE I. Relation between acidity of gastric juice and slow spike frequency

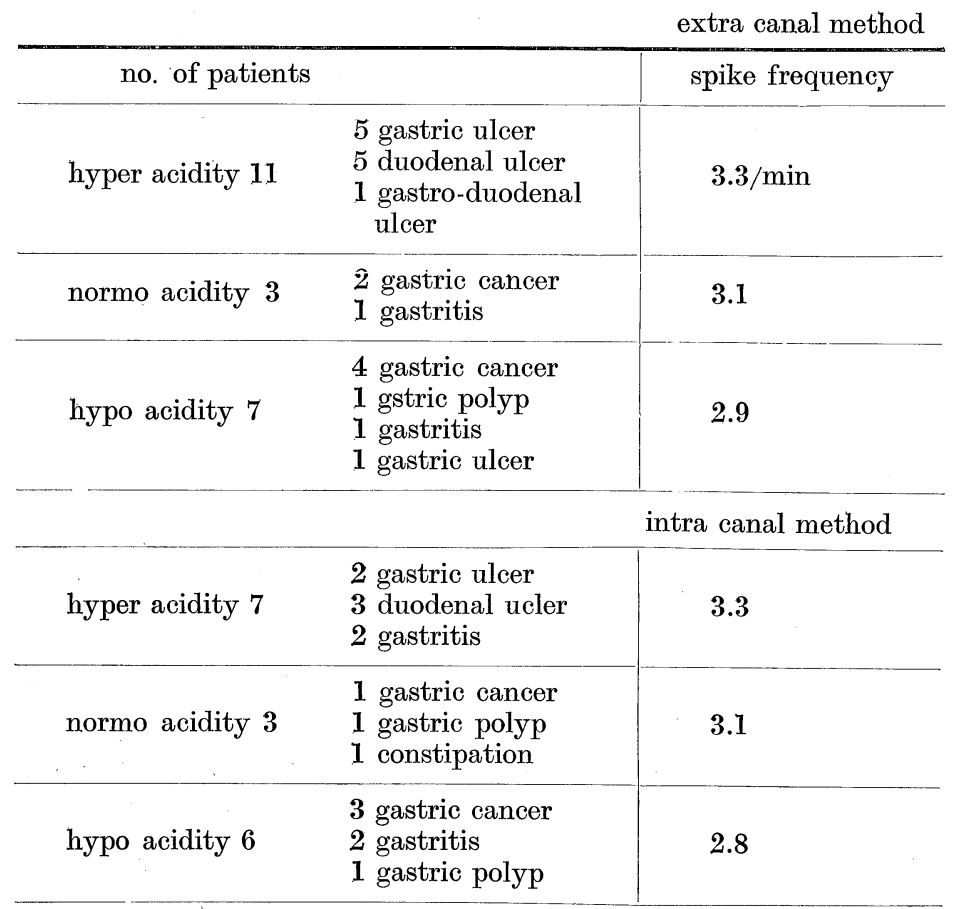

the rationality of author' $\mathrm{s}^{6)}$ considering the function of stomach as the whole "gastric function".

Next, the changeability of the frequency was studied when the anesthesia was changed to ether anesthesia the spike frequency was decreased. However, even the ether anesthesia had only slight effect on the electrogastromyogram, if the consistency was low and the time was short. In other words the changeability of the spike frequency is relatively low. Namely, the stability of the frequency is relatively high. And this is the one of the reasons why the authors ${ }^{7}$ consider the analysis of the spike frequency must be useful to study the gastric function of the patients individually. When stronger agents, for exmaple gastric cooling as a treatment for duodenal ucler, was administrated, the frequency of the spikes decreased remarkably. And if the stomach is frozen, the spike disappears.

\section{INTRA CANAL Electrogastromyography}

Next problem is per oral intra canal electrogastromyography. The authors call it electrogastrography for short.

In 1960 the intra canal electromyography of the stomach was brought into success in experiment with canine stomach by the authors, suggesting clincial availability in meeting shortcomings of the ancient balloon method to examine the gastric motility. In 1963 the first clincial application of the intra canal 
electrogastromyography was carried out successfully (using a suction electrode). Prior to clincial application, various types of electrode had been tested on the 48 canine stomachs. And then a suction electrode has been chosen. The electrode could be inserted into the stomach of the patient through his mouth without any operative procedure. At first the somewhat thick gastric suction tube was improved. The bipolar electrodes were set around the suction hole at the tip of the tube. The gastric mucosa $\mathrm{w}$ as to be sucked in and fixed at the hole. And later a set of electrode with tripc arity instead of bipolarity has been used so that two of three poles could be selected for bipolar leading. As the $\mathrm{S} / \mathrm{N}$ ratio is much influenced by the contact or direction of the electrodes, the tripolarity has three times of chances more than bipolarity to get good contact and good direction of the electrodes. And at present the tube has some elastic bend for fitting the contour of greater curvature so that the insertion into the antrum can be easily done. Moreover, the electrode has a small flash lump in its tip to show the location of it through the abdominal wall. The electrode is connected to the suction pump. A pump running continuously at $200 \mathrm{mmHg}$ is most suitable, but a rubber suction ball or large syringe with a locking unit is suitable for ordinary use.

The amplifier has been produced specially as an electrogastrograph. The block diagram is shown in Fig. 2. The gain is selected in 3 sensitivity ranges which are calibrated to indicate $200 \mu \mathrm{V}$ on the recording paper at 10,5 and 2.5 $\mathrm{mm}$. And the speed of the recording paper is $120 \mathrm{~mm}$ per minutes. The amplifier has two filters. One is a twin T type cutting off at $50 \mathrm{c} / \mathrm{s}$, and the other is a low pass filter with a cut off frequency of $40 \mathrm{c} / \mathrm{s}$ and $6 \mathrm{~dB}$ octave curve.

A typical pattern of electrogastrogram which was recorded by the new electrogastrograph is shown in Fig. 3. As described above, the electromyogram

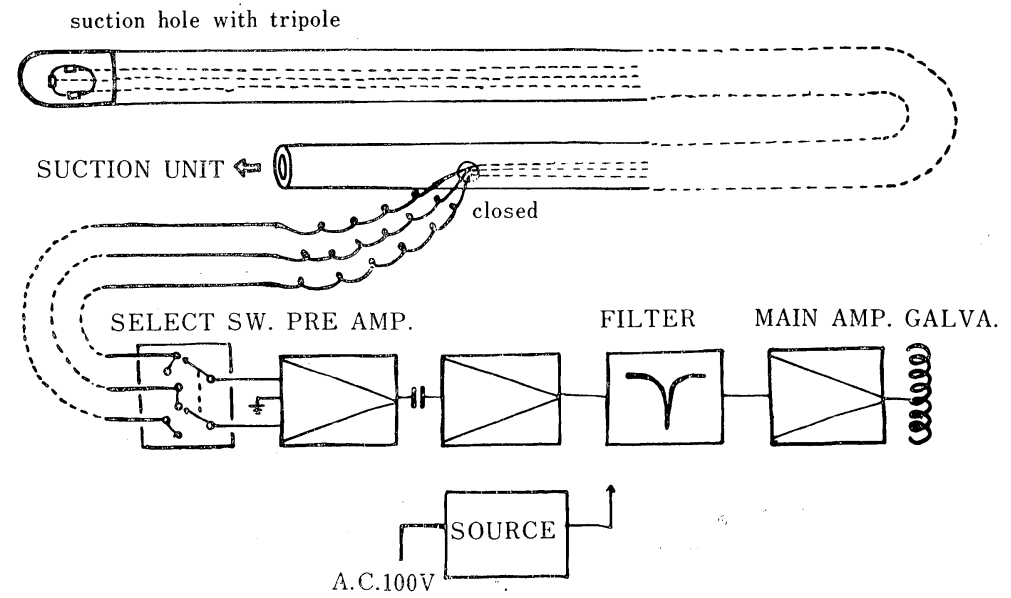

FIt. 2 Block diagram of the electrogastrograph and schema of the suction electrode 


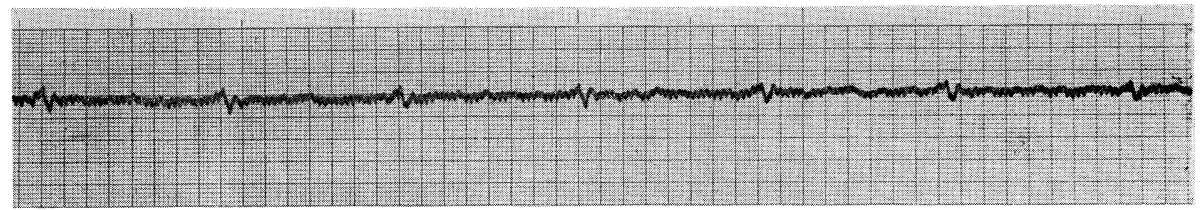

H.S. 29 yrs, female, gastric cancer

FIG. 3 A typical pattern of intra canal electrogastromyogram (electrogastrogram).

of the human gastric smooth muscle consists of simple, rhythmical and slow spike like potentials. And as Fig. 3 shows the slow spikes of intra canal electrogastrogram consist with the spikes recorded by extra canal method.

The lowering of the $\mathrm{S} / \mathrm{N}$ ratio can not be avoided on the intra canal method. While, the jamming of ECG has not so much interference because of the difference of the frequency with each other. When the interference caused by breath is occurred the examiner tells the patient to hold the breath shortly. Then the spike of electrogastrogram becomes to be able to discriminative. Even if the $\mathrm{S} / \mathrm{N}$ ratio falls into minus, the discrimination is possible.

On the intra canal electrogastrography the examined cases have been reached 61 patients with various gastric diseases. The measured value must be analysed again.

The mean value of the amplitude of the spikes was $660 \mu \mathrm{V}$, and duration was 1.9 seconds. On the intra canal method these values are more unreliable than that of extra canal method. However, the spike frequency is considered to be reliable. The frequencies distributed from 2.0 to 4.0 per minutes and the mean value was 3.0 per minutes. While it was 3.2 on the extra canal method.

Being similarly to the extra canal method, on the intra canal electrogastrogram the frequency had a tendency being parallel with gastric acidity (Table 1). In the cases of duodenal ulcer therefore the frequency was 3.4 per minutes, which was higher than in the cases of gastric cancer whose frequency was only 2.7.

This time, the test of fluothane anesthesia was carried out with similar results with ether anesthesia, having depressive effect on the spike frequency. Then, the investigation on the changeability of the frequency has been applied for testing of drug effect. For example, the frequency was increased by the administration of bethanechol chloride and decreased by metoclopramide. These two drugs are known to have a positive effect upon the gastric evacuation. However, this result shows the difference of effective point of each drug. In addition to the results described above in experimental and clinical studies the frequency was increased by injection of a number of cholinergic drugs, and by anoxia and by filling of the stomach with hot water. And it was decreased by injection of anti-cholinergic drugs, adrenaline, chlorpromazine and succinylcholine and by gastric cooling or freezing. However, the frequency was uninfluenced by pethidine or pentothal 
TABLE 2. Influence of various agents upon spike frequency

\begin{tabular}{|c|c|}
\hline spike frequency is & agents \\
\hline increased by & $\begin{array}{l}\text { acetylcholine inj. } \\
\text { bethanechol inj. } \\
\text { prostigmin inj. } \\
\text { trimethyl GABA methylester inj. } \\
\text { anoxia } \\
\text { hot water filling }\end{array}$ \\
\hline decreased by & $\begin{array}{l}\text { adrenalin inj. } \\
\text { atropine inj. } \\
\text { pyrodifenium bromide inj. } \\
\text { valethamate bromide inj. } \\
\text { metoclopramide inj. } \\
\text { chlorpromazine inj. } \\
\text { s.c.c. inj. } \\
\text { gastric cooling } \\
\text { gastric freezing } \\
\text { ether anaesthesia } \\
\text { fluothane anaesthesia }\end{array}$ \\
\hline uninfluenced by & $\begin{array}{l}\text { pethidine inj. } \\
\text { pentothal sodium inj. } \\
\mathrm{N}_{2} \mathrm{O} \text { anaesthesia }\end{array}$ \\
\hline $\begin{array}{l}\text { not consistently } \\
\text { influenced by }\end{array}$ & vagotomy \\
\hline
\end{tabular}

sodium injection and $\mathrm{N}_{2} \mathrm{O}$ anesthesia. Moreover the frequency was not consistently influenced by vagotomy. This fact shows the control of the nerve upon the gastric smooth muscle is not so strong as it is considered before. And the agent which is considered to have a direct effect upon the smooth muscle seems to have stronger effect than the agent which acts through the nerve. The results are arranged in Table 2.

\section{Comment}

The investigation of these characteristics has indicated the value of electrogastrography in patho-physiologic research and the selection of the most appropriate form of the treatment of gastric disorder. And the authors feel that in the near future, the sharp band pass filters and sure feed back systems will be developed and by using monopolar method, the analysis of the amplitude and the shape of the gastric slow spikes will become more easy and reliable. Theil the electrogastrography will be more popular method to study the gastric function clinically.

\section{References}

1. Bozler, E.: The action potential of the stomach. Amer. J. Physiol., 146, 693, 1945.

2. Code, C.F. et al.: A concept of control of gastrointestinal motility by the pace setter potential. Digest of the seminar on clinical application of smooth muscle electromyography. Honolulu, Hawaii, 1968. 
3. Daniel, E.E. and Irwin, J.: Electrical activity of gastric musculature. Handbook of Physiology, section 6, Vol. 4, P. 1969, American Physiological Society, Washington, 1968.

4. Bass, P. et al.: Electrical activity at the gastroduodenal junction. Amer. J. Physiol., 201, 587, 1961.

5. Oi, M. and Tanaka, Y.: Clinical application of the intracanal gastroelectromyography. Digest of the 6th international conference of medical electronics and biological engineering, Tokyo, Japan, P. 482, 1965.

6. Oi, M. Tanaka, Y. and Twai, Y.: Electrogastromygoraphy. Digest of the 7th international conference of medical and biological engineering. Stockholm, Sweden, P. 335, 1967.

7. Tanaka, Y. and Yanagisawa, K.: The electromygoraphy for the stomach and its clinical application, Japanese Journal of Medical Electronics and Biological Engineering, 1, 309, 1963. 


\title{
Electromyographic Studies on the Mechanism of Pylorospasm Following Vagotomy
}

\author{
TSUNEO SHIRATORI, M.D. \\ Department of Surgery, Tohoku University School of Medicine, Sendai, Japan
}

We have been studying the nature of the motor function of the stomach in various conditions with the use of cinefluoroscopy, electromygoraphy and intraluminal pressure study.

I would like to discuss the problem whether vagotomy causes pylorospasm followed by delayed gastric emptying as has long been believed by a number of investigators. As indices of pylorospasm, a high incidence of anti-peristaltic discharges and prolonged discharge intervals are available in the electromyography.

\section{Materials and Methods}

Adult mongrel dogs weighing about $10-15 \mathrm{~kg}$ were used for the experiments. The animals, with their stomachs emptied by 24 hours fasting, were fixed in supine position and anesthetized with thiopental-sodium administered intravenously. They were then laparotomized by median incision.

Experiment 1: We made an experiment in which the transection-anastomosis of the stomach along the border line between the corpus and antrum with or without preservation of the vagal nerve fibers. Two bipolar silver-electrodes were used for the recording of electromyogram. One electrode was fixed on the anterior wall of the stomach $1 \mathrm{~cm}$ proximal to the pyloric ring and close to the greater curvature. Another electrode was fixed $4 \mathrm{~cm}$ proximal to the pyloric ring.

Normo-peristaltic discharge was assumed to occur when the action potentials were first recorded by the proximal electrode and anti-peristaltic discharge was said to have occurred when the action potentials were first recorded by the distal electrode.

Experiment 2: In the next place, the truncal vagotomy was made to clarify what will happen if truncal vagotomy alone is performed. This experiment was observed electromyographically and grossly.

Experiment 3: We studied the gastric motility with the use of electromygoraphy before and after truncal vagotomy with instillation of warm water of various amount. Bilateral truncal vagotomy was performed immediately above the diaphragm, and special attention was paid not to leave any vagal branches 
to the stomach. The duodenum was ligated at about $10 \mathrm{~cm}$ distal to the pyloric ring to prevent outflow of water.

Next, $200 \mathrm{ml}$ of water was instilled via the naso-gastric tube into the stomach in 300 seconds, and the procedure was repeated every 30 minutes until $1,000 \mathrm{ml}$ was given in each case before and after vagotomy, and the changes of the gastric motility were investigated grossly and electromyographically.

Experiment 4: The present study deals with the effect of local distension of the stomach on the motor function of the pyloric portion. Motor function of the pyloric portion was observed electromyographically with two electrodes on the serosal aspect of the pyloric portion of the stomach. The rubber balloon attached to tip of a nasogastric tube inserted via mouth was inflated with air to distend each part of the stomach.

\section{Results}

Experiment 1: Fig. 1 shows the result of transection of the stomach including the vagal nerve fibers. Before transection, normo-peristalsis occurred in the distal portion of the stomach at the rate of 97 per cent and anti-peristalsis 3 per cent. After transection along the border line between the corpus and antrum, the rate of normo-peristalsis was reduced down to 42 per cent, while that of anti-peristalsis was increased to 58 per cent. Besides, it was noted grossly that the peristaltic movement of this antral area became much more intense.

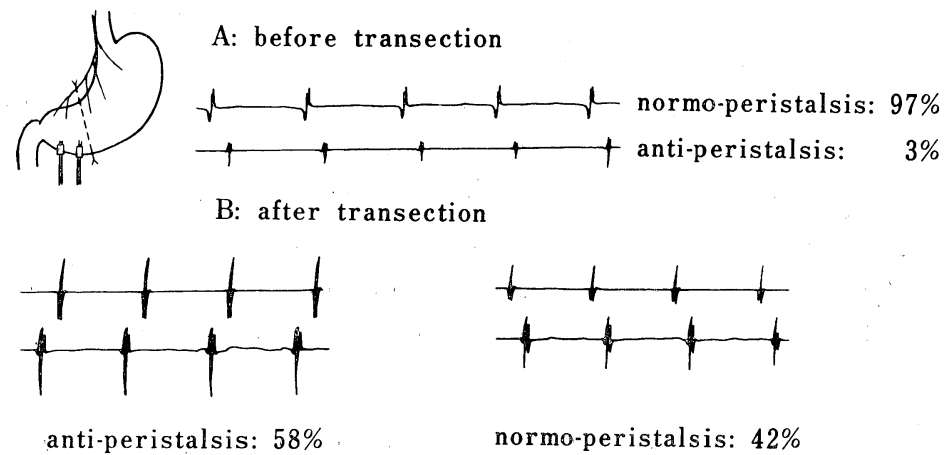

FIG. 1. Gastric transection without vagal preservation

In another group of dogs, we performed the transection at the same level as before but carefully preserved the vagal fibers which run along the lesser curvature.

As Fig. 2 shows, 99 per cent of peristalses were normo-peristaltic before transection which reduced to 49 per cent after such transection of the stomach. The result was more or less similar to that of transection without vagal preservation 1 
and intesne peristaltic movement was also observed in the portion distal to the line of transection-anastomosis.

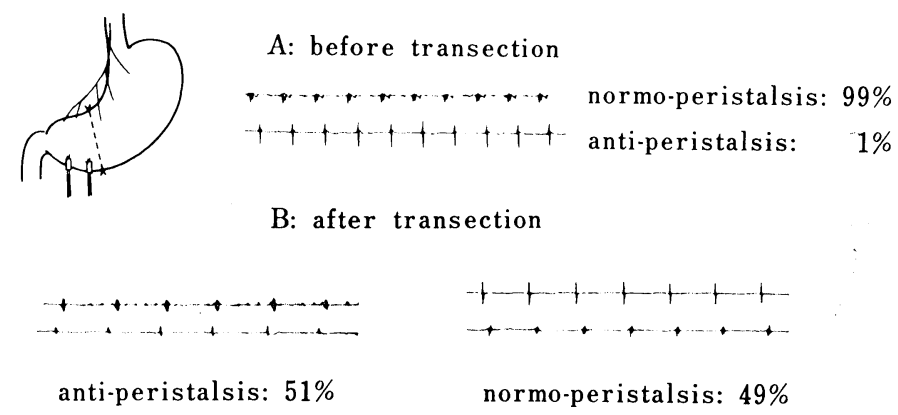

FIG. 2. Gastric transection with vagal preservation

From the view point of the discharge interval of normo-peristalsis, similar result was obtained. The discharge interval of normo-peristalsis in the distal portion of the control stomach was 15.4 sec. on the average. Following transection-anastomosis, the discharge interval of normo-peristalsis prolonged to 31.8 sec. when the vagus nerves were preserved. This value was rather equal to 34.6 sec. of the case of vagus nerve section.

Out of these experiments, it is easily understood that the transection of the stomach, either with or without vagal preservation, results in the same change of peristalsis; i.e., increased rate of anti-peristalsis, and prolongation of the discharge interval. ${ }^{1)}$ Addition of vagotomy did not change the perstalsis signfiicantly.

Experiment 2: Following truncal vagotomy, there occurred shortening of the discharge interval, and reduced velocity. Grossly, the stomach became hypotonic with slight dilatation, and peristaltic movement became somewhat weak. As far as the stomach is empty, there is no spastic contraction of the pylorus after vagotomy. Therefore, it could be concluded that the truncal vagotomy of the empty stomach does not cause pylorospasm as has been believed in general.

On the other hand, all of us know clincially that the truncal vagotomy without any drainage procedures results in a delayed gastric emptying which seems to contradict the present conclusion. To fill this gap, we performed Experiment $\left.3 .^{2}\right)$

Experiment 3: In the normal canine stomachs with $200 \mathrm{ml}$ of warm water inside, $95 \%$ of the persitalses were normo-peristaltic. When the amount of warm water reached $600 \mathrm{ml}$, there occurred some anti-peristalses. With $800 \mathrm{ml}$ inside, about $50 \%$ of the peristalses were anti-peristaltic.

Similar procedure was performed on the vagotomized stomachs. Significant percentages of anti-peristalses developed first with $800 \mathrm{ml}$ of warm water requiring $200 \mathrm{ml}$ more than non-vagotomzied stomachs to develop the same percentage of 
anti-peristalsis. This difference may be referred to the reduced tonus of the gastric wall induced by vagotomy, requiring much more water to increase the intragastric pressure for occurrence of anti-peristalsis.

The incidences of anti-peristalsis following vagotomy are lower than those before vagotomy except for $1,000 \mathrm{ml}$ case where the values are almost equal.

We shows the change of the discharge intervals of the electric activity of the pyloric canal before and after vagotomy with gradual instillation of warm water into the stomach. Before vagotomy, the discharge interval prolonged in proportion to the amount of warm water. On the contrary, vagotomzied stomach failed to show prolongation of the discharge interval until the amount of warm water reached $600 \mathrm{ml}$, following which the stomach showed a sharp rise with further instillation of warm water.

These findings seem to suggest that the vagotomized stomach became hypotonic with suppressed peristaltic movement, being unable to react to the distension by water instillation up to $600 \mathrm{ml}$ in amount. However, when the amount of warm water exceeded $600 \mathrm{ml}$, vagotomized stomach re-gained excitability, resulting in a strong peristaltic movement with frequent anti-peristalses particularly in the pyloric portion.

Another experiment (Experiment 4) was performed, in relation to intragastric pressure study, on dog stomach to elucidate the reason why the motor function of the pyloric portion increases markedly when excessive amount of warm water is instilled into the stomach.

Experiment 4: The result when each part was alternately distended with the same amount of air is shown in Table 1. With insufflation of the same amount of air, the motor function of the stomach in respect to the discharge interval and propagation velocity of normo-peristalsis increased most when the pyloric portion was distended; and distention of the corpus and fundus was followed by less marked excitation of the pyloric portion in the decreasing order. The incidence of antiperistaltic discharges was highest with $13.2 \%$ when the pyloric portion was distended.

TABLe 1. Sites of distension with $50 \mathrm{ml}$ of air and electromygographic findings

\begin{tabular}{l|c|c|c}
\hline \multirow{2}{*}{} & \multicolumn{2}{|c|}{ normo-peristalsis } & anti-peristalsis \\
\cline { 2 - 4 } & $\begin{array}{c}\text { discharge } \\
\text { interval (sec) }\end{array}$ & $\begin{array}{c}\text { propagation } \\
\text { velocity (mm/sec) }\end{array}$ & incidence (\%) \\
\hline control & 14.2 & 12.1 & 0 \\
fundus & 15.8 & 12.4 & 1.2 \\
corpus & 16.6 & 12.8 & 0.2 \\
pylorus & 19.7 & 13.9 & 13.2
\end{tabular}


The latter part of the experiment consisted of observation of the motor function when a balloon in the chosen part was insufflated gradually. The discharge interval of normo-peristalsis showed a tendency of marked prolongation with even a slight increase in amount of insufflated air when the balloon was placed in the pyloric portion. Furthermore, the propagation velocity of normoperistalsis showed the same tendency; that is, marked increase of the propagation velocity with even slight increase of distention of the pyloric portion. On the other hand, corporic and fundic distention was followed by less marked response than that of pyloric distention, although the response of corporic distention was greater than that of fundic distention.

As in Fig. 3, with pyloric distention the incidence of anti-pertalsis was high; for instance $45.8 \%$ with $100 \mathrm{ml}$ of air. Meanwhile, the incidence of antiperistalsis was lower with fundic and corporic distention in spite of increasing amount of air.

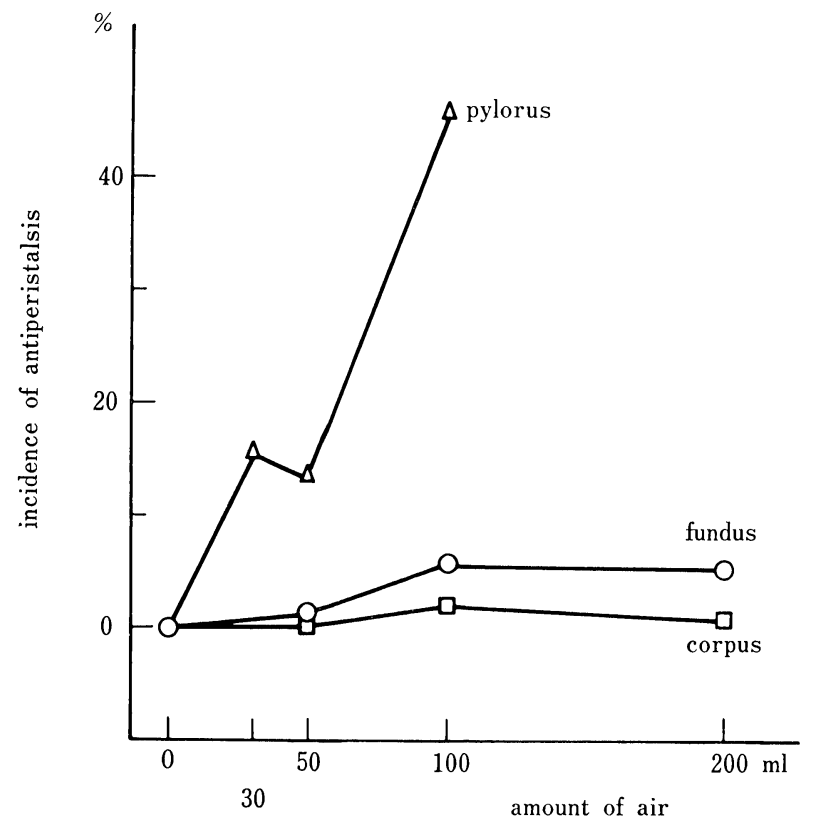

Fra. 3. Relationship between incidence of anti-peristalsis and amount of air in each part.

From this experiment, it was found that the pyloric portion was quite sensitive to dilating distension and a slight increase of dilating distension caused activation of peristaltic movement. It was also found that marked dilatation of the pyloric portion caused a spasm-like abnormal excitation of the portion with resultant development of frequent anti-peristalsis and large propagation velocity. 
These findings seem to elucidate the phenomenon observed in the abovementioned water-instillation experiment. It is now clear that the spasm-like abnormal excitation of the pyloric portion occurred through dilating distention of the pyloric portion when excessive amount of water was instilled.

\section{SƯMMARY}

In summary, the followings were learned out of a series of the present experiments;

1) Transection-anastomosis of the stomach, particularly along the border line between the corpus and antrum, caused predominant anti-peristalsis, strong peristaltic movement, and spastic contraction in the portion distal to the line of transection. These findings are quite uniform regardless whether the vagal nerve branches to the distal portion of the stomach are preserved or not. Therefore, transection of the stomach wall, but not the section of the vagus nerves, is the primary factor of the pylorospasm.

2) Truncal vagotomy which was performed on the empty stomach without gastric transection induces hypotonicity, decreased peristaltic movement, and frequent normo-peristalses; but least likely to develop pylorospasm. The mechanism of pylorospasm after vagotomy is explained as follows: Atonic dilatation occurs first with resultant retention of the stomach content. This retention and increased intragastric pressure distend the pyloric portion and cause markedly increased motor function of the pyloric portion. This is pylorospasm.

In other words, pylorospasm following vagotomy occurs secondarily to atony and marked dilatation of the stomach.

\section{References}

1. Tsuneo Shiratori, Shun Kuroda, Kyoji Sugawara and Tetsuhiko Hatafuku: Effect of vagotomy or splanchnicectomy on the motility of the canine stomach with transection-anastomosis. Tohoku J. exp. Med., 93, 317-329, 1967.

2. Ken Nagaoka: Electromygoraphic study on the mechanism of delayed gastric emptying after vagotomy in dogs. Tohoku J. exp. Med., 95, 1-13, 1968. 


\title{
Some Experiment and Clinical Aspects of the Ureteral Function
}

\author{
SEIGI TSUCHIDA, M. D. \\ Department of Urology, Tohoku University School of Medicine, Sendai, Japan
}

\begin{abstract}
Described below are the findings obtained from the study of the ureteral function using conventional electrodes and our newly devised electrode for recording electroureterograms (E.U.G.) through a cystoscope.
\end{abstract}

\section{Structure of Electrode and Method of Introduction}

In the present experiment, the electrodes for extracellular introduction were used. However, we have devised the following electrode in addition to the conventional needle-shaped electrode.

a) 'U-loop' collar electrode for intraureteral introduction (Fig. 1-a): A silver wire ring $0.3 \mathrm{~mm}$ in diameter is fixed around an F-5 ureteral catheter $3 \mathrm{~cm}$ from the tip, and a second ring is placed around the catheter $1 \mathrm{~cm}$ away from the first one to form a bipolar electrode. This distance is adjustable. A hole is bored in the catheter on either side of the electrodes and $1 \mathrm{~cm}$ from them. The distance between the two holes is thus $3 \mathrm{~cm}$. A piece of nylon thread is inserted into the catheter from the base, pulled out through the first hole, pushed in again through the second hole and fixed to the catheter at the tip.

When this catheter has been inserted into the ureter through a cystoscope, the nylon thread is pulled at the base of the catheter and the part of the catheter

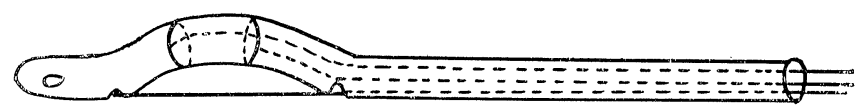

b

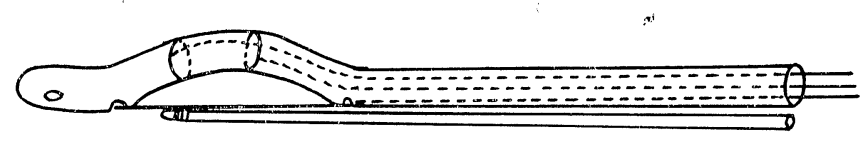

Frct. 1 Structure of U-loop collar electrode (a), and instrument for combination of electroureterography and urometry (b) 
to which the electrodes are fixed bends in an arc-shape to make close contact with the ureteral wall.

b) Structure of instrument for synchronized recording of electroureterogram and urometrogram: Since the intraluminal pressure of the ureter changes periodically in accordance with the peristalsis, it is important to obtain synchronized recording of the electroureterogram and urometrogram. For this purpose, the instrument shown in Fig. 1-b has been devised. This instrument is a combination of the 'U-loop' collar bipolar electrode just described and an F-3 polyethylene ureteral catheter.

For accurate recording of the action potential and the variation of the urometrogram at the same position in the ureter, the F-3 ureteral catheter is connected to a transducer.

Fig. 2 shows the electroureterogram recording synchronized with the urometrogram. The top line is the urometrogram and that shown below is the electroureterogram.

a

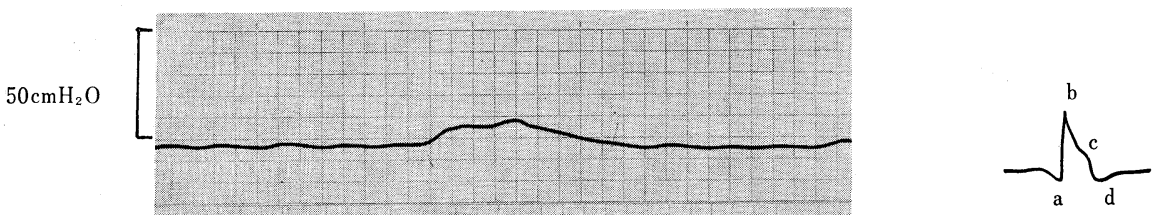

$1 \mathrm{mV}$
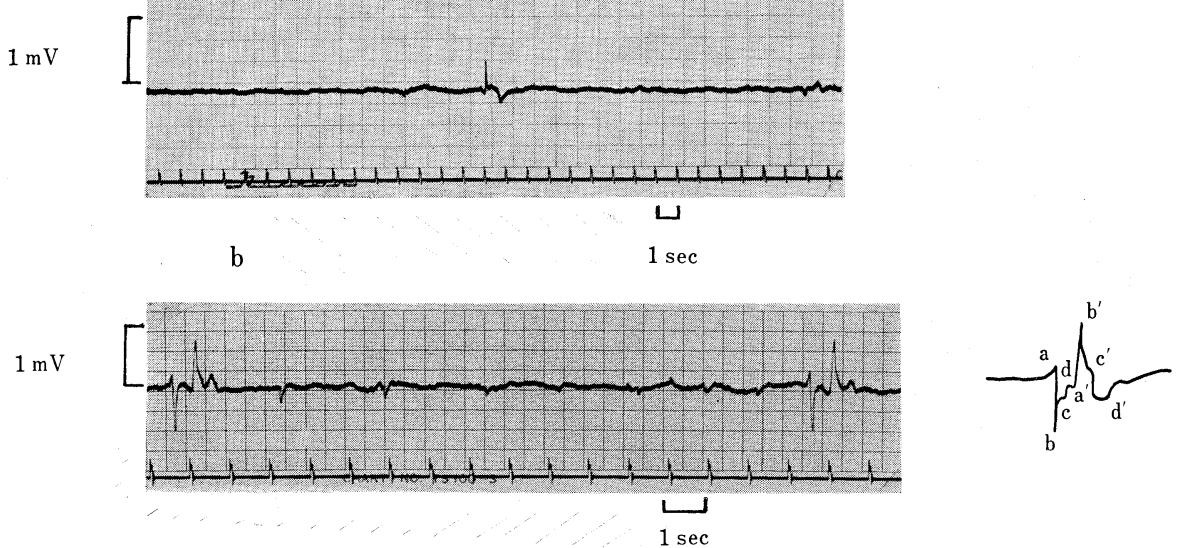

Fic. 2 Electroureterogram obtained from human ureter a, unipolar lead [simultaneous recording of urometrogram (upper) and electroureterogram (lower)]; b, bipolar lead.

\section{Amplitude of Electroureterogram, Discharge Interval and Travel Rate}

The action potential recorded by the 'U-loop' collar electrode was identical to that recorded by Sleator \& Butcher ${ }^{1)}$ and Shiratori et al. ${ }^{2)}$ as shown in Fig. 2-a. 
(a) A positive wave appears first, this is followed by the appearance of (b) a sharp negative main potential and then (c) a negative accessory potential and (d) a positive after potential. In the bipolar lead, this is followed by the reciprocal deflections $a^{\prime}, b^{\prime}, c^{\prime}$ and $d^{\prime}$ (Fig. 2-b).

Normal values observed in the electroureterogram obtained from the introduction of the 'U-loop' collar electrode include, in human subjects, an amplutide of $0.13-1.04 \mathrm{mV}$, average $0.48 \mathrm{mV}$, discharge interval of 6-34 sec., average 17.0 sec., travel rate of $20-90 \mathrm{~mm} / \mathrm{sec}$, average $30 \mathrm{~mm} / \mathrm{sec}$.

\section{Movement of Peristalsis from Renal Pelvis to Ureter}

For the purpose of clarifying how the urine is conveyed from the renal pelvis to the urinary bladder, a study has been made by means of the needle electrode and the 'U-loop' collar electrode.

When the action potential of both the renal pelvis and the ureter was recorded in this state, the action potential recorded in the renal pelvis was also recorded in the ureter without being lost in the pelvi-ureteric juntion. From this fact, it can be said that the peristalsis of the renal pelvis and the ureter is conveyed at the ratio of $1: 1$. However, when the renal pelvic pressure was raised by infusing physiological saline solution through an F-6 vinyl tube by nephrostomy, an increase in the action potential was observed in the renal pelvis. However, this action potential was not completely conveyed but was partially blocked in the pelvi-ureteric junction i.e. the discharge ratio in the renal pelvis and the uretr can be said to be $2: 1$ or $3: 1$.

However, observation of the peristalsis transferred to the ureter, by means of the intraureteral introduction method, revealed that the peristalsis travelled to the extravesical ureter without disappearing.

We made a physiological examination of the intramural ureter which runs obliquely through the wall of the urinary bladder to its orifice in the trigonum. An attempt was made to record an electroureterogram in this portion, but no action potential was recorded

Based on these findings, the urine conveyance can be explained as follows: ripple-like peristalsis which is observed in the renal pelvis does not positively convey the urine but plays only an auxilliary role. The chief role is played by the peristalsis of the extravesical ureter between the pelvi-ureteral junction and the point where the ureter enters the wall of the urinary bladder. Finally, the lower part of the ureter near the bladder makes a positive contraction to raise the intraluminal pressure which ejects the urine into the urinary bladder through the intravesical ureter like a waterpistol. 


\section{Some Factors Influencing Ureteral Peristalsis}

a) Control by the nerve: In order to clarify this problem, we made an electroureterogram while removing the influence of the extraneous nerve. The kidney and ureter of a dog were exposed, separated from the connecting tissues and isolated. The renal artery and vein were then cut and anastomosed completing autotransplantation. Thus, electroureterograms before and after the operation could be obtained. The urine flow increased from a preoperative level of $0.4 \mathrm{ml}$. to one of $0.5 \mathrm{ml} .30$ minutes after the anstomosis and the action potential was recorded distinctly. The postoperative discharge interval extended to 5-6w/min. after the operation as compared with 10-12 w/min. before the operation, but the amplitude was identical.

An attempt was made next to increase the urine flow by administering mannitol solution to the case on which the autotransplantation had been performed, but it showed a reactive tendency quite similar to the normal control group. When the nephrostomy was performed and physiological saline solution was infused into the renal pelvis through the nephrostomised region the discharge interval shortened, showing a reaction identical to normal. From the above results, it can be said that ureteral peristalsis is not influenced at all by the extraneous nerve.

b) Control by the blood vessels: An attempt was made to record electroureterograms while blocking the blood vessels controlling the ureter for the purpose of studying their influence. Ligation was first performed on the renal artery and vein, the urine flow stopped but the action potential continued to appear. Although the discharge interval lengthened to $5-6 \mathrm{w} / \mathrm{min}$. as compared with 7-8 $\mathrm{w} / \mathrm{min}$. before the ligation was performed, the nature of the peristalsis did not change.

c) Electroureterograms at changing urine flow: Some recent studies have claimed that when the urine flow increases the interval between ureteral peristalsis shortens. Others have contradicted this view by claiming that in this condition the interval becomes prolonged. We made some investigations into this problem.

Interval diagrams of both discharge intervals and travel rates at changing urine flow were recorded oscilloscopically through a computer.

At a fixed urine flow of $0.3 \mathrm{ml} / \mathrm{min}$., action potentials were emitted regulary at definite discharge intervals although in limited instances somewhat irregularly. However, even in the latter instances discharge intervals changed in steps rather than at random, thereby indicating that several different paces existed (Fig. 3).

As for changes associated with increase in urine flow, the discharge interval altered in various ways according to the idiosyncrasy of the dog, i.e. ureteral disposition and degree of urine flow. In an ureter showing action potentials with definite discharge intervals, an increase in urine flow from 0.5 to $2 \mathrm{ml} / \mathrm{min}$. gave rise to a reduction in dischage interval from 4.7 to 3.2 sec., 3.0 sec. later: this was followed by the onset of a definite pace. At the same time the travel rate dropped 

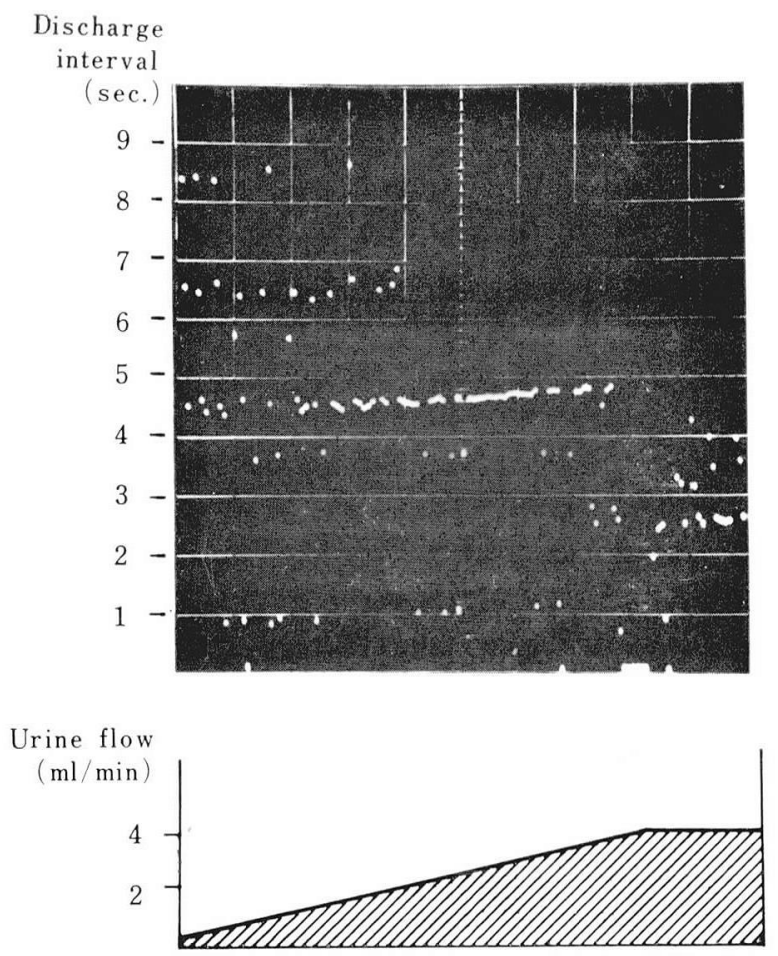

FIG. 3 Diagram of discharge interval on gradual increase of urine flow in the case of varying paces.

from 20.0 to $15.0 \mathrm{~mm} / \mathrm{sec}$. and thereafter remained essentially unchanged.

The dog was then given an intravenous infusion of a $13 \%$ mannitol solution to increase urine flow to approx. $15 \mathrm{ml} / \mathrm{min}$. About 5 minutes after the increase of urine flow, a decrease in discharge interval from 3.5 to 3.1 sec. occurred although intervals subsequently tended to reach approx. 4.2 sec.

As can be seen in Fig. 4, in response to the increase in urine flow from 0.5 to $5 \mathrm{ml} / \mathrm{min}$., for one dog, a drop of the discharge interval from 5.2 to 3.8 sec. was recorded. This was followed by a gradual prolongation from 5.0 to 5.2 sec. However, a subsequent rapid increase of the urine flow to $15 \mathrm{ml} / \mathrm{min}$., by intravenous infusion of mannitol, resulted in the reduction of the discharge interval to 3.2 sec. When urine flow was kept constant at a higher level, the discharge interval again become prolonged and returned to the previous value.

By contrast, in ureters having action potentials with varying paces (Fig. 3), the action potentials appeared at discharge intervals of 3 different paces, i.e. a' $(=8.5$ sec.), $\mathrm{a}^{\prime \prime}\left(=6.5 \mathrm{sec}\right.$ ) and $\mathrm{a}^{\prime \prime \prime}(=4.5 \mathrm{sec}$.), when the urine flow was as low as 0.3 $\mathrm{ml} / \mathrm{min}$. In response to a gradual increase in urine flow to $5 \mathrm{ml} / \mathrm{min}$., paces a', $\mathrm{a}^{\prime \prime}$ 

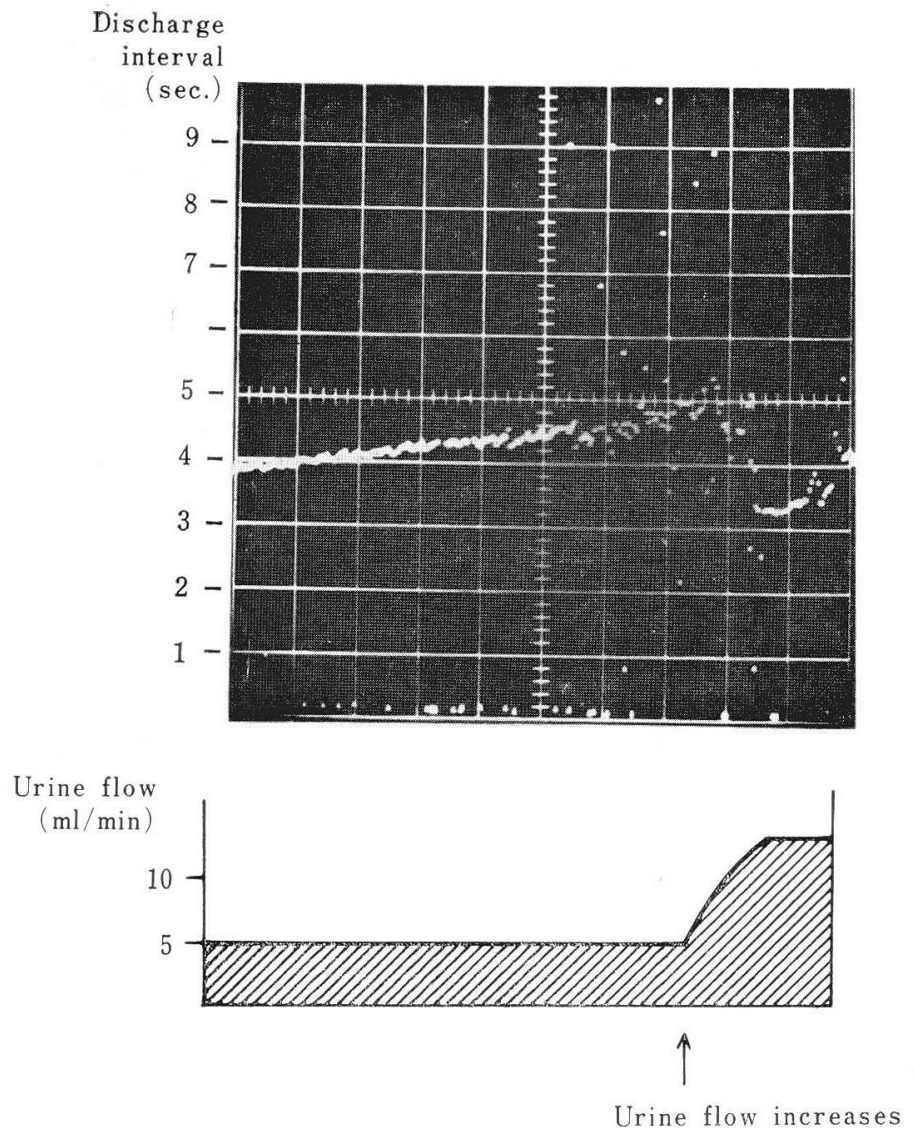

FIG. 4. Diagram of discharge interval on sudden increase of urine flow.

and $\mathrm{a}^{\prime \prime \prime}$ all disappeared and were replaced by shorter paces of about 2.5 sec. in most instances.

Another dog displayed a drop of discharge interval from 4.5 to 3.2 sec. as urine flow increased from 0.5 to $1.8 \mathrm{ml} / \mathrm{min}$. When the urine flow was then reduced to as little as $0.3 \mathrm{ml} / \mathrm{min}$., the discharge interval became gradually prolonged to a maximum length of 5.8 sec. and an interval of approx. 5.2 sec ensued.

It follows from these findings that there is a definite pace in the ureteral peristalitic movement and that the movement is always inclined to assume that pace. In addition, depending on the rate of increase of urine flow two phenomena can be observed. With the gradual increase of urine flow the discharge interval shortens gradually. With the sudden increases of urine flow the intervals drop suddenly to a different pace. It is very interesting that when the discharge interval paces were not constant increase in the urine flow resulted in the convergence of the discharge intervals to the shortest possible pace. 
d) Reaction of ureteral mucosa: From what has already been made clear, it seems that the peristalsis of the ureter is delicately influenced by changing the urine flow. Yet, whether the peristalsis is brought about by the stretching of the muscle cells caused directly by the rise of the intraureteral pressure or whether first the membrane is affected by direct contact with the urine and transmits the pressure to the muscle cells, has not been clarified.

To solve this problem, studies have been made to see the change in the ureteral function when in vivo and in vitro application of an anesthetic agent, xylocaine, was made separately to the mucosal membrane, the muscle layer and the serous membrane.

For the experiment, an adult dog was used and the injection of xylocaine was performed as follows: The kidney was exposed, a nephrostomy was made and an F-3 ureteral catheter was inserted into the renal pelvis through the fistula thus formed so that the tip of the catheter approximated to the end of the ureter.

$5-10 \mathrm{ml}$ of $4 \%$ xylocaine solution were injected into the renal pelvis and the ureter, then the electromyogram of the renal pelvis and ureter was obtained with needle-shape bipolar electrodes. The action potential disappeared immediately after the injection of the solution. This state, though it differed depending on the dose of the solution, lasted from 2 to 3 minutes to some 10 minutes. Thereafter an action potential of very small amplitude appeared, returning to the original state after 2-3 minutes.

The F-3 ureteral catheter was then inserted into the ureter from the bladder, xylocaine solution was injected into the lower part of the ureter, and the electropyelogram and electroureterogram were recorded in the upper region of the ureter. A normal action potential was recorded.

As already outlined above, the effect of anesthetizing the membrane of the renal pelvis and the ureter became apparent in an extremely short time. To study its effect on the serous membrane, a piece of guaze soaked with xylocaine solution was placed around the ureter and an electroureterogram was taken. However, in sharp contrast to the above case, no effect was observed.

A further attempt was made to record the electroureterogram by performing an intramuscular injection of xylocaine, but no change was observed whatsoever 2-3 minutes after the injection, though the amplitude of the action potntial became smaller by degrees, eventually disappearing. The disappearance of the wave is believed to be due to the infiltration of xylocaine solution to the membrane.

Results of in vitro experiments: An isolated canine ureter was used for the experiment, and xylocaine was made to act on the serous membrane as described below. The isolated ureter was fastened to a supporting rod in a thermostatic bath of a total capacity of $30 \mathrm{ml}$, filled with Krebs solution and bubbled with $5 \%$ carbonic acid gas and $95 \%$ oxygen to record the change in the tesile strength. When it was necessary to make xylocaine affect the ureteral mucosa, both ends of the 
ureter were connected to a polyethylene tube and Krebs solution was perfused from one end of the tube at a constant speed. Xylocaine was added to the solution.

The application of tensile strengrh ranging from 1-2 gr. to the ureter gave rise to spontaneous excitation 10-20 minutes afterwards with a gradual increase in the contraction frequency, returning to a constant and regular rhythm 20 minutes after the start of the contraction.

Xylocaine was added to the extraureteral Krebs solution to increase its concentration and no marked influence of xylocaine was observed even when the concentration was raised by $10^{-6}$. But, when the concentration was further raised by $10^{-5}$, an increase in the discharge frequency was observed by a slight decline in the amplitude was noticed.

When the concentration was then raised by $10^{-4}$, the amplitude showed a marked reduction, leaving a fine contraction curve and this was soon followed by the disappearance of the contraction.

In the intraureteral perfusion experiment, phenomena identical to those in above experiment were seen. Xylocaine shows an in vitro inhibitory action on the spontaneous contraction of the isolated ureter, but its action on the surface of the serous and the mucosal membranes is almost the same.

From the above results, the membrane can be said to play an important role in vivo occurrence of ureteral peristalsis but this is lost as a result of the excision of the ureter.

When we think of the above differnce between in vivo and in vitro experiments, it appears that the supply of blood through the vessels plays an important role in view of the fact that the influence of the extraneous nerve can be disregarded, as was shown in the experiment of autotransplantation.

\section{Clinical Application of Electroureterogram}

The recording of E.U.G. on several clinical subjects through the cystoscope revealed abnormal findings including congenital hydronephrosis, nephroureterolithiasis, pyelonephritis and blind ureter.

\section{References}

1. Butcher, H.R.Jr. \& Sleator, W.Jr.: A study of the electrical activity of intact and partially mobilized human ureters. J. Urol., 73, 970, 1955.

2. Shiratori, T., Katayose, K., Suetake, Y., Kawamata, S., Kinishita, H., Takakura, K., Enokido, K., Shiga, R., Nomura, T., Usuba, G. and Chiba, K.: Electromyographic studies on urinary tract. 1. Fundamental studies on EMG of animal and human ureters. Tohoku J. Exp. Med., 69, 175, 1959. 


\section{Action Potentials of the Rectum by Balloon Method}

KENZO MATSUOKA, M.D.

Department of Surgery, Osaka University School of Medicine, Osaka, Japan.

Nowadays, it is not difficult to record action potentials from the digestive tract. Action potentials from the human esophagus or the stomach have been reported by various methods. There are, however, few reports on the electromyogram from the rectum and sigmoid colon. The first reliable article on the action potentials of the human rectum was published by Suzuki and Ueda in 1956, according to the information of the author. Their work, however, may not be known, as it was published in Japanese. I would first like to introduce their investigation, then add my own supplementary work in the present paper.

This investigation consists of two parts; that is, experimental study and clincial application.

\section{Materials and Methods}

Rabbits were used to examine the efficiency of the recording electrode. Clinically, normal subjects, patients with Hirschsprung's disease, spinal lesions and other digestive tract disease were examined.

The recording electrode is a platinum wire $0.3 \mathrm{~mm}$. in diameter and coated with resin insulator. A specific electrode holder was devised which consisted of a small rubber plate with a few cone shaped knobs $3 \mathrm{~mm}$. high. The platinum wire was passed through the center of the cone to its top and cut off. The stump of the wire was the electrode tip. This electrode holder was fastened to a rubber balloon with a silk string. The rubber balloon, about $3 \mathrm{~cm}$. in diameter, was connected to a 12 to $7 \mathrm{~mm}$. rubber tube. Air was instilled through the tube to the balloon through a small hole in the wall of the tube, and the balloon was inflated to the desired size after insertion into the sigmoid colon or the rectum. The wires were connected to a penwriting electromyograph. An indifferent electrode was placed on the anterior edge of the iliac crest. So-called unipolar and bipolar recordings were performed. The other end of the rubber tube was connected to a mercury manometer or a kimograph.

\section{Results and Discussion}

1. Animal Experiment

For use in the rabbit, a smaller electrode apparatus was produced. Multiple 
spikes were observed simultaneously when peristalsis of the bowel occurred visually. Each of the spikes was much different in shape but the amplitudes were in the range of 30 to $200 \mu \mathrm{V}$ and the durations were 5 to $30 \mathrm{msec}$. The spikes were monophasic, biphasic and polyphasic and these patterns were intermingled. These spikes usually constituted a spike burst. It appeared difficult to identify these spikes as true action potentials from the bowel, and an effort was made to solve this problem. The results were as follows.

(A) Optimum time constant and interpolar resistance: Optimum time constant was 0.05 sec. The fluctuation of the basic line appeared when the time constant was 0.2 sec. and increased at 1.5 sec. Interpolar resistance was within 100 kilohms for bipolar recording and 50 kilohms for so-called monopolar recording when the contact between the electrode and the mucous membrane was tight. This resistance was considered as one of the best indicators for screening the spike burst.

(B) Friction and movement of the electrode: Contact between the electrode and the mucous membrane was not always good by this method. When friction and movement of the electrode was induced by pulling the rubber tube, giant voltage polyspikes, several to ten times larger than the normal voltage, appeared and the baseline was often scaled out. Slight movement of the wire did not effect the action potential, but with moderate movement, spike bursts, which apparently did not differ from true action potentials, appeared. This time, however, the recording from two monopolar leads synchronized. This phenomena is not observed in true action potentials.

When the abdominal pressure was increased, the internal pressure of the balloon increased but spikes were not observed. On the contrary, increase in internal pressure and spikes are seen almost simultaneously with normal peristalsis. Such artifacts were exmained in various conditions and it was finally recognized that in many conditions there are indicators by which we can discriminate the artifact from the true spikes.

\section{Clinical Study}

In the normal human subject, the action potentials of the rectum could hardly be recorded, because peristalsis is stilled in these parts under normal conditions. To obtain the action potential, peristalsis was activated by intramuscular injection of prostigmine and spike bursts of high amplitude $(100$ to $200 \mu \mathrm{V})$ were observed.

The balloon electrode was inserted in the colon of the patient with Hirschsprung's disease. Low amplitude voltage waves (5 to $100 \mu \mathrm{V}$ ) sparsely appeared, but spike bursts were never observed with prostigmine activation in the narrow portion of the rectum. In the dilated portion, the normal peristaltic pattern of spikes was recorded and the spastic contraction pattern sometimes intermingled. These results give direct evidence that the distal narrow segment of the intestine is 
inert and fails to transmit waves of peristalsis from the proximal normally innervated bowel in this disease. That the defect is in the distal normal appearing gut and not in the proximal hypertrophied colon is functionally demonstrated.

Patients treated by Swenson's operation have frequent bowel movements for one to three weeks after the operation and then gradually recover. The action potentials from the bowel show parallel activity according to the patient's condition.

Other patients with various digestive diseases and spinal lesions were exmained and data was almost successfully obtained.

\section{Summary}

The action potentials of the sigmoid colon and the rectum were successfully recorded without any operative procedure by Suzuki's balloon method. This method was applied in various functional disorders of the abdomen and has been used to obtain the valuable data. I believe this method is applicable for clincial use. 


\title{
Studies on the Constitution of Muscle Layers of Stomach of Canine and Human
}

\author{
KAN YOSHIDA, M.D. \\ Department of Surgery, Jikei University, School of Medicine, Tokyo, Japan
}

We have studied the anatomical structure of the gastric musculature. The anatomy of the gastric-muscle should bear an importance, because the gastric electromyogram which is so controvertial as discussed in this seminar, originates from the muscle.

Initially it was observed that there was difference of the electrical potential in accordance with different portion of the stomach body, angulus, antrum and pyloric ring.- and it was strongly suggested that this electrical change is caused by the difference among muscle structure of the gastric portions.

At first we made microscopic examination, but we failed to understand the gross structure of the musculature. Then, we have macroscopically observed the muscle structure which was free from the mucosal layer (Fig. 1). The specimen of the Figure 2 is a human whole stomach.

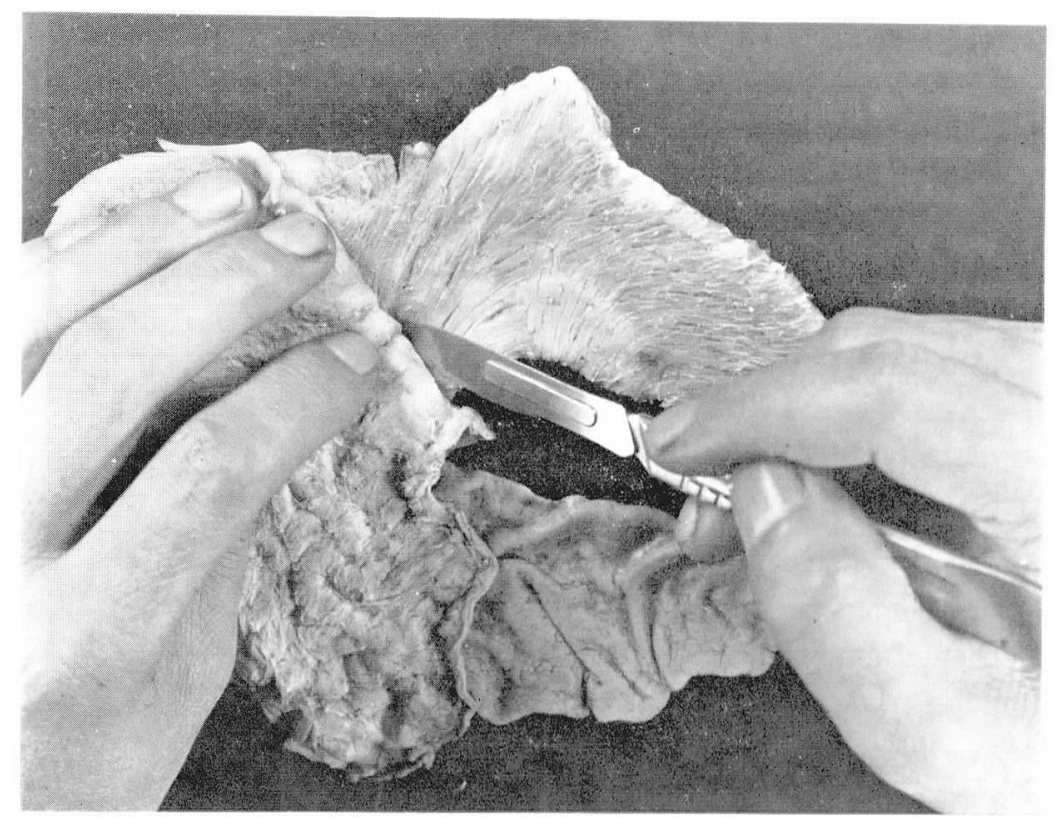

Fic. 1. The mucosal layer is separated from the muscle coat in each specimen with scalpel through the submucosa, exposing the inner surface of the musculature. 


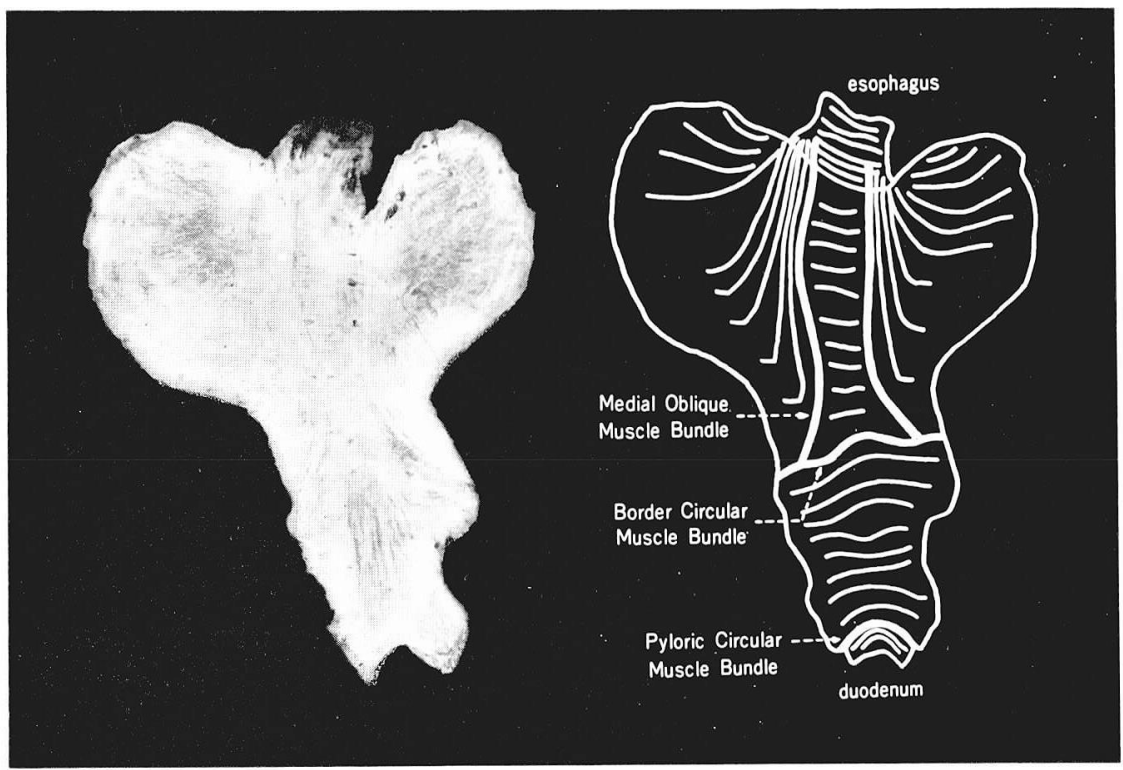

Frc. 2. The oblique muscle and the circular muscle (human).

The oblique muscle originates from the esophageal circular muscle and get united with the circular muscle of the body (Fig. 2). The oblique muscle bundle which exists near the lesser curvature on the anterior or posterior wall, is the longest oblique muscle bundle. We named this bundle the "anterior or posterior medial oblique muscle bundle." There is no oblique muscle distal to the circular muscle bundle with which both the medial oblique muscle bundles connect. Therefore, we consider this circular muscle bundle as the border between the body and the antrum on the musculature, and we named this the "border circular muscle bundle" (Fig. 2).

Next, I refer to the muscle of the fundus. This portion consists of 2 muscle layers; one is of the longitudinal muscle and the other is of the circular fiber having the center at the fornix of the fundus, and has no relation with the esophageal muscle. There is a problem whether this muscle belongs to the oblique muscle layer or the circular muscle layer. Presently this muscle is considered as special muscle that does not belong to any at these layers (Fig. $2 \& 4$ ).

Now, the longitudinal muscle. The longitudinal muscle originates from esophageal longitudinal muscle. However, at the lesser curvature, on or near the gastric angulus, there is no or only a few longitudinal muscle fibers (Fig. $3 \& 4$ ).

It has been known that there are 3 muscle layers of the stomach. However, in our observation the most portions of the stomach has 2 muscle layers, and especially the part of the anglus has only one layer of the circular muscle (Fig. $3 \& 4$ ).

Concerning to these findings of the human stomachs, the canine stomachs was observed too. But there are 2 points of difference between human and canine 


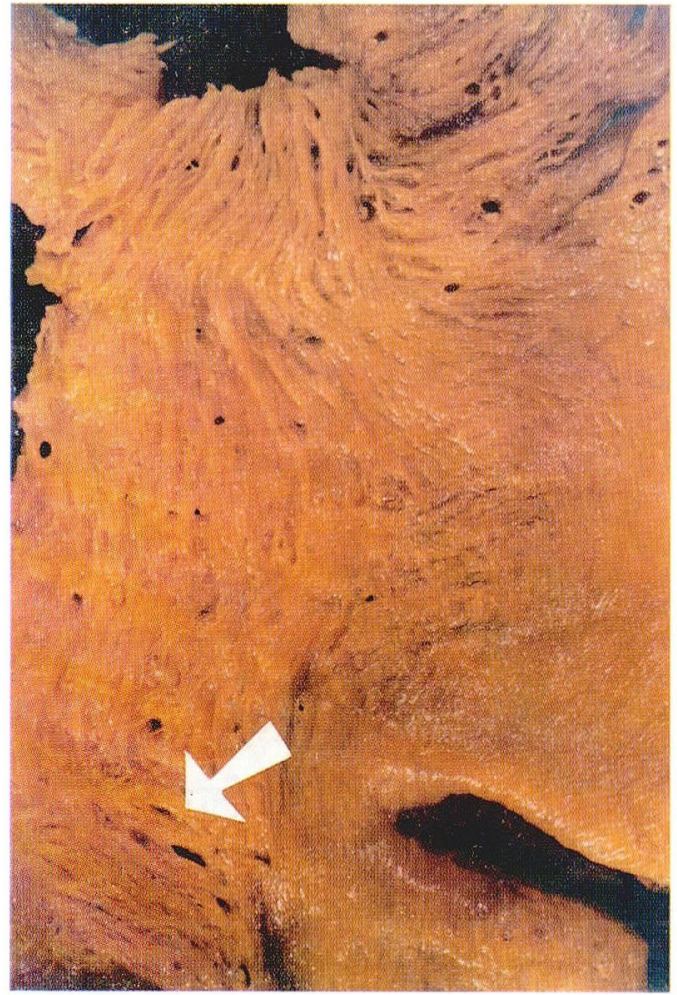

FIG. 3. At the lesser curvature, near the gastric angulus, there is no longitudinai muscle fibers (human).

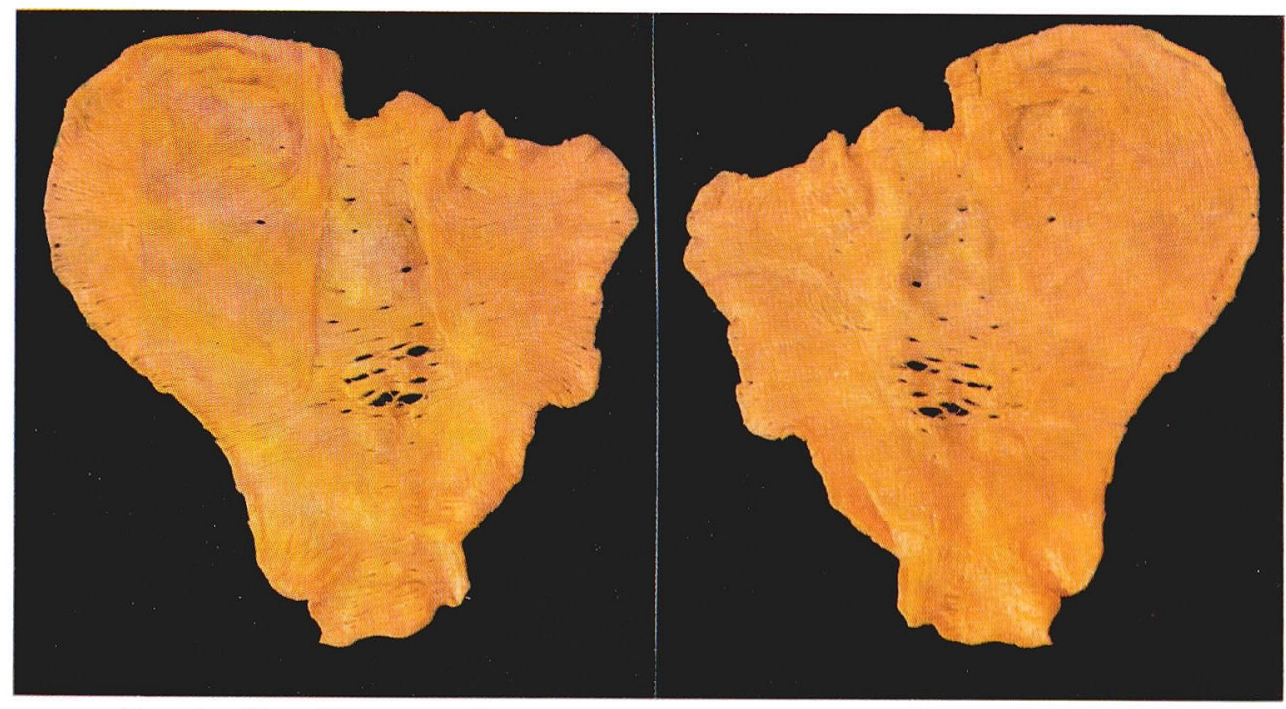

FIG. 4. The oblique muscle and circular muscle (left), and the longitudinal muscle (right).

The specimen (canine) of the muscle layer is treated with solution $\left(20^{\circ}\right)$ of $\mathrm{HNO}_{3}$ to remove the connective tissue from muscle. 
stomachs.

First, in human cases, the muscle fibers form a bulky bundle, while they do not in the dog, they form only a fine bundle (cf. Fig. 1 \& Fig. 4).

Second, in human cases, the bulk of the circular muscle of the pyloric ring is so much more prominent at any other part. But in the canine cases, the pyloric circular muscle is not so remarkable.

Recently we have treated the specimens of the muscle layer with solution $(20 \%)$ of $\mathrm{HNO}_{3}$ for 3 to 7 days to remove the connective tissue from the muscle.

This technique has made the inspection of the muscle fibers much easier than before. Figure 4 shows the specimen so treated.

\section{References}

1. Kan Yoshida: Studies on the constitution of muscle layers of human stomach. Part I: The constitution of muscle layers of surgically resected gastric specimens. Jap. J. Smooth Muscle Res. 1, 80-94, 1965.

2. Kan Yoshida: Studies of the constitution of muscle layers of human stomach. Part II: The constitution of muscle layers of stomach and its relation to the location of gastric ulcers. Jap. J. Smooth Muscle Res., 1, 95-104, 1965. 